\title{
REDRESSING THE ROLES OF ANTHOCYANIN PIGMENTS IN VEGETATIVE AND REPRODUCTIVE ORGANS
}

\author{
BY \\ LUKE JAMES COONEY
}

\begin{abstract}
A thesis
submitted to the Victoria University of Wellington in fulfilment of the requirements for the degree of Doctor of Philosophy
\end{abstract}

Victoria University of Wellington

(2015) 



\section{Abstract}

Anthocyanin pigments are common in both reproductive and vegetative organs in plants, yet their functional significance is not entirely understood. While communicative functions have received considerable attention in reproductive organs and the role of anthocyanic colouration in frugivore and pollinator attraction is well understood, it has also been suggested that anthocyanins provide a communicative function in vegetative organs i.e. it may be that anthocyanic colouration in leaves deters herbivores by signalling a plant's defensive investment. Conversely, there is evidence that anthocyanins in vegetative organs perform a number of physiological functions such as photoprotection and mitigation of various environmental stressors. While these physiological roles have received considerable attention in leaves, little is known about the applicability of these functions to anthocyanins in reproductive organs. There is evidently a gap in anthocyanin research; no study has provided unequivocal support for a communicative function for anthocyanins in vegetative organs and no study has shown that anthocyanins perform a physiological function in the reproductive organs in any species other than domesticated crop plants. To address this imbalance in anthocyanin research my thesis tested for a signalling role in vegetative organs, and then investigated a physiological role for anthocyanins in reproductive organs.

In chapter two, I hypothesised that for Pseudowintera colorata, red (anthocyanic) leaf margins reduce leaf herbivory by signalling to herbivorous insects the presence of increased chemical defences. Using a natural population of $P$. colorata, I showed that leaves with the wider red margins contained higher concentrations of anthocyanins and polygodial, a sesquiterpene dialdehyde with known anti-feedant properties, and incurred less natural herbivory. Additionally, laboratory feeding trials involving a natural P. colorata herbivore, Ctenopseustis obliquana larvae, showed a preference for green-margined leaves over red, but only when feeding trials were conducted under light regimes which allowed discrimination of leaf colour. Collectively, my data show that red leaf margins provide a reliable and effective visual signal of chemical defence in $P$. colorata. Moreover, $C$. obliquana larvae apparently perceive and respond to the colour of leaf margins, rather than to olfactory cues. My study is therefore the first to provide direct support for a communicative function for anthocyanins in vegetative organs.

In peduncles, rays and pedicels, the sterile components of an inflorescence, anthocyanin accumulation has exclusively been considered an adaptation to promote frugivore visitation; 
however, anthocyanins may instead be produced to mitigate light stress. In chapter three, I tested the requirements of a physiological function, that anthocyanins provide photoprotection for Sambucus nigra peduncles which turn red prior to fruit maturation. I found that accumulation of red pigmentation required exposure to full sunlight and that anthocyanins significantly reduced the quantity of green light that would normally reach chlorenchyma in the peduncle. Under saturating white light, red peduncles maintained higher quantum efficiencies of photosystem II compared to green peduncles, and red portions of peduncle recovered from photoinactivation more quickly than did green portions. My data are, therefore, the first to show that anthocyanins perform a physiological function in the reproductive organs of a naturalised species.

In chapter four, I hypothesised that anthocyanin accumulation in senescing Sambucus canadensis peduncles prolongs senescence and enhances nitrogen resorption. Red peduncles possessed several traits indicative of a prolonged senescence; their rates of chlorophyll and xanthophyll decline were lower, while tensile strength and elasticity were higher than for green peduncles. Red peduncles were also less susceptible to photoinactivation than the green ones at the later stages of senescence. However, manipulating green peduncles with light filters possessing transmittance properties comparable to an anthocyanic tissue layer did not increase peduncle longevity or nitrogen resorption. I concluded that like senescing leaves, red peduncles display many characteristics indicative of a prolonged senescence, but I am unable to attribute this benefit to the presence of anthocyanins.

This thesis provides a significant contribution to our understanding of the role of anthocyanins in plants in two ways: it is the first to directly demonstrate that anthocyanins perform a communicative function in vegetative organs, and is the first to show for a naturalised (non-cultivar) species, that anthocyanins perform a physiological function in reproductive organs. 


\section{Acknowledgements}

First and foremost, I would like to thank my primary supervisor, Kevin Gould, for the guidance and support I have received. Kevin provided a constant source of inspiration and this thesis would not have been possible without his encouragement, patience and enthusiasm. I would also like to acknowledge the academic and scientific advice I received from Kevin Burns, Iggy Menzies, Niky Hughes, John van Klink, Nigel Perry, Amy Johnson and in particular, Martin Schaefer and Barry Logan. Martin and Barry have been especially helpful, providing considered and thoughtful feedback when requested and making me feel truly welcome while hosting me at their respective institutions. I would also like to thank those members of the Gould and Davy labs, Karl Yager, Gagendeep Jain, Maheshini Mawalagedera, Kate Calcott, Emma Gibbin and Shaun Wilkinson for providing a stimulating work environment.

This thesis was the product of a great deal of practical assistance. I would like to thank Michael Walsh for his dedication to this project and the countless hours he spent working in the field and laboratory. My time in Maine would not have been so productive and enjoyable had it not been for your help. Bart Cox and Wendy Jacobs, thank you both for making your homes available and allowing me to greedily harvest your elderberry. Much appreciation is owed to Anne Barrington for raising larvae and John van Klink, Nneka Nnatubeugo and Jaret Reblin for their expertise on the HPLC and the hard work each provided.

This research was funded by a Victoria University of Wellington Doctoral Scholarship, a PGSA research excellence award and FSRG travel award provided by Victoria University of Wellington, and grants made by the Royal Society of New Zealand Marsden Fund and the German Academic Exchange Service (DAAD).

Finally, I would like to thank my family - Grace for your support and partnership, Mum, Dad, Jim and Maureen for the encouragement and frequent financial assistance and Jade for the inspiration to keep on working hard. 


\section{Table of Contents}

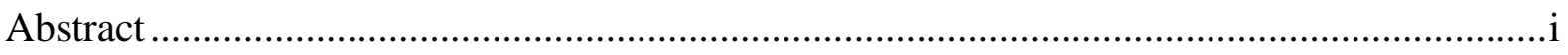

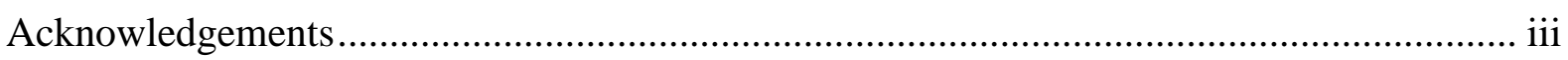

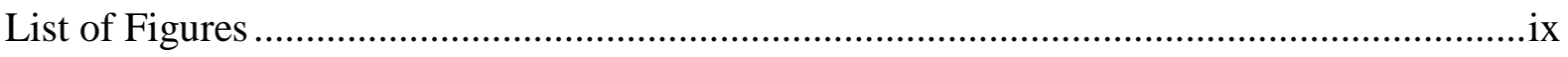

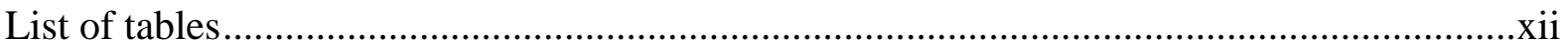

Chapter 1: Redressing the roles of anthocyanins in vegetative and reproductive organs -

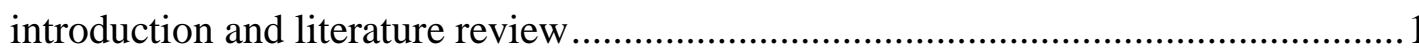

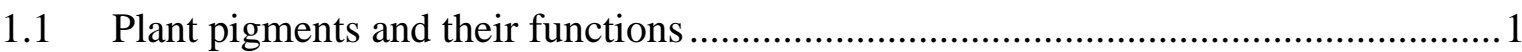

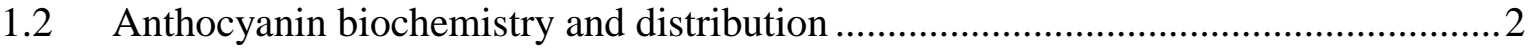

1.2.1 Biochemical and spectral properties of anthocyanins.......................................2

1.2.2 The histological, morphological and temporal distribution of anthocyanins ......4

1.3 Physiological significance of anthocyanin accumulation ......................................

1.3.1 Photoinhibition and its prevention by anthocyanins .....................................

1.3.2 Criticisms of the photoprotective hypothesis............................................ 8

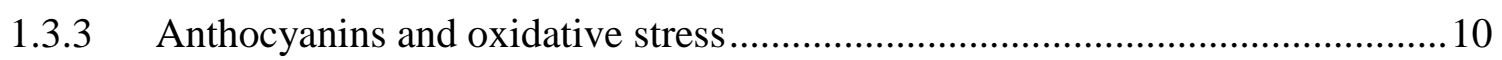

1.3.4 Anthocyanin accumulation in juvenile leaves ............................................... 11

1.3.5 Anthocyanin accumulation in senescing leaves............................................. 12

1.3.6 Criticism of the nitrogen resorption hypothesis .............................................. 13

1.3.7 Does anthocyanin accumulation prolong senescence? .................................... 14

1.3.8 Other putative physiological roles for anthocyanins ................................... 15

1.3.9 Physiological function in reproductive organs ............................................. 16

1.4 Communicative functions in vegetative organs ............................................... 20

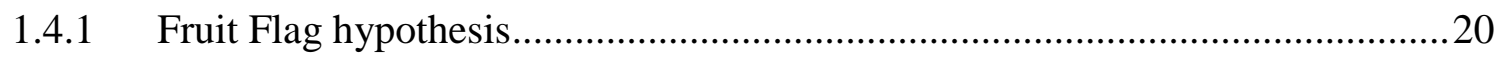

1.4.2 Anthocyanic pigmentation undermines herbivorous insect camouflage ...........21

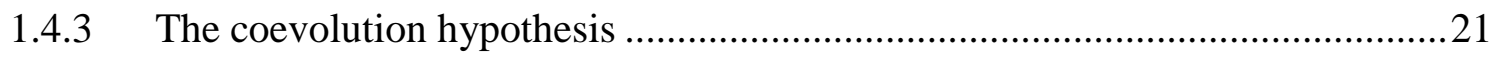

1.4.4 Coevolution prediction 1: Are red leaves less palatable? .................................22

1.4.5 Coevolution prediction 2: Does leaf colour affect aphid behaviour? ................23 
1.4.6 Coevolution prediction 3: Does signalling leaf palatability provide a selective advantage?

1.4.7 Those signalling roles purportedly subsumed by coevolution..........................25

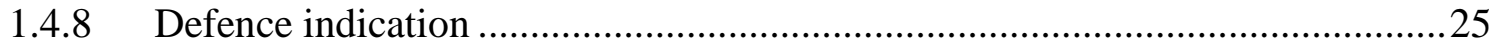

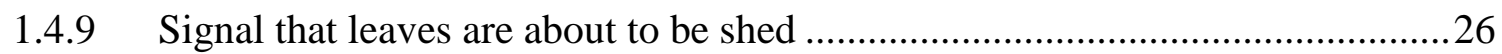

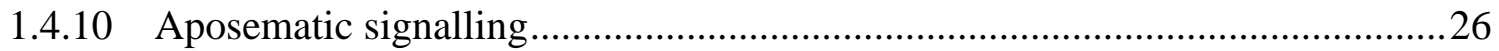

1.4.11 Extension of the coevolution hypothesis to non-senescing organs...................27

1.5 Addressing the dichotomy in anthocyanin research...........................................28

1.5.1 Pseudowintera colorata is a model species to test for a communicative function

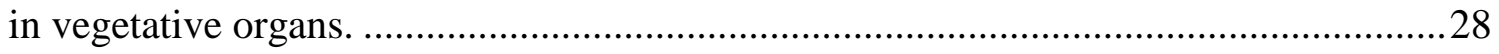

1.5.2 Sambucus spp. are the model species to test for a physiological function in reproductive organs.

Chapter 2: Red leaf margins indicate increased polygodial content and function as visual signals to reduce herbivory in Pseudowintera colorata ............................................ 31

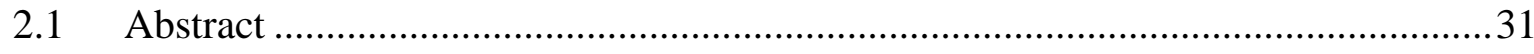

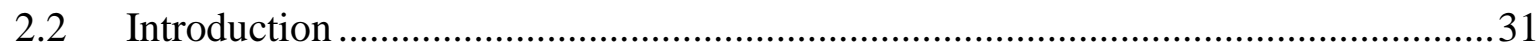

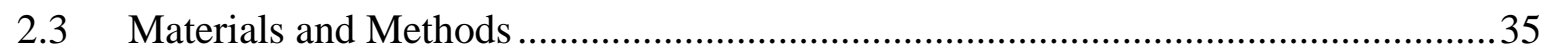

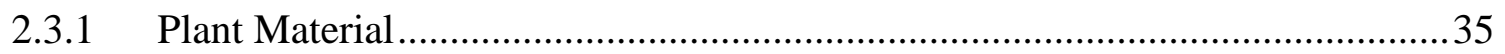

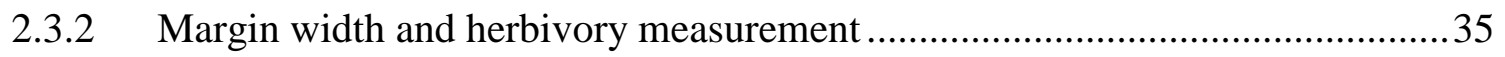

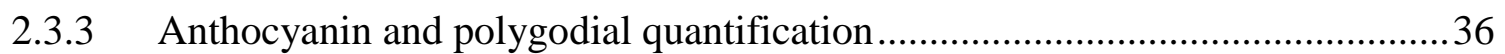

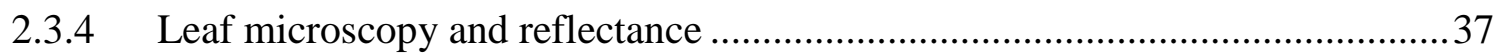

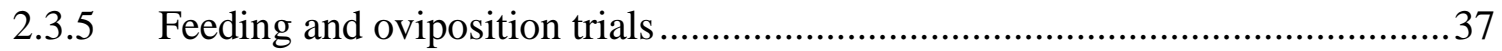

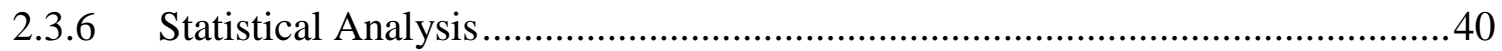

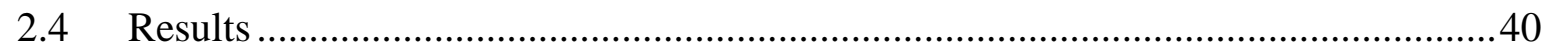

2.4.1 Pseudowintera colorata margin pigments .................................................. 40

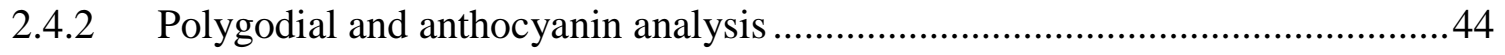

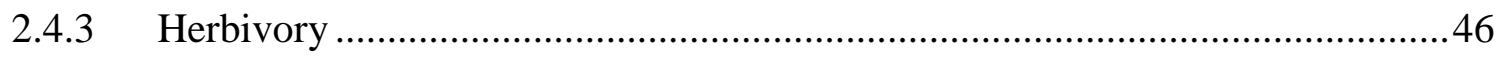

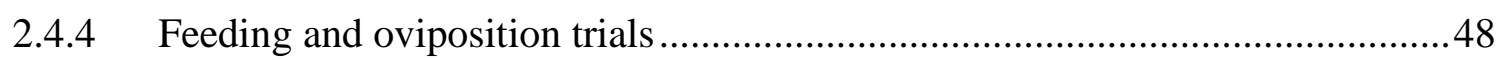


2.5.1 Defensive investment is concentrated at the margin.....................................52

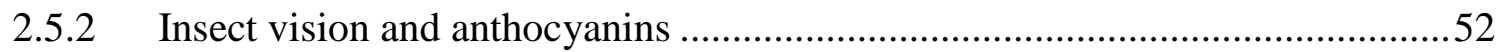

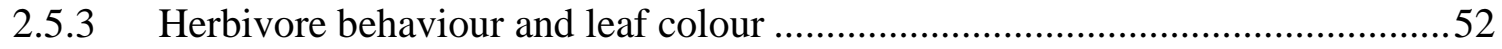

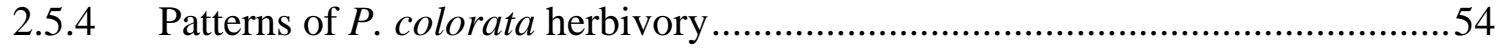

2.5.5 The adaptive significance of red leaf margins in $P$. colorata ...........................55

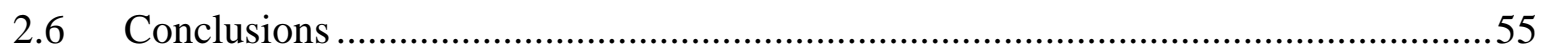

Chapter 3: Anthocyanins perform a photoprotective function in peduncles of Sambucus nigra

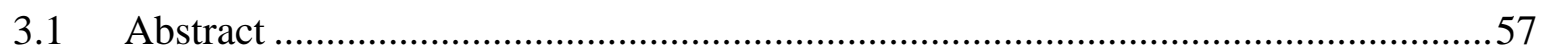

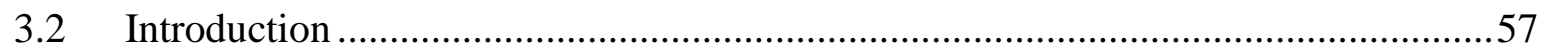

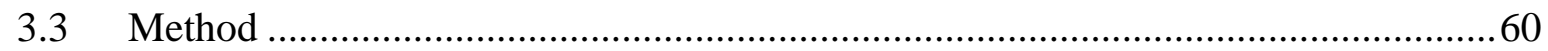

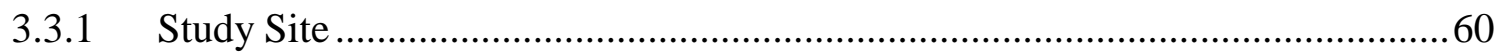

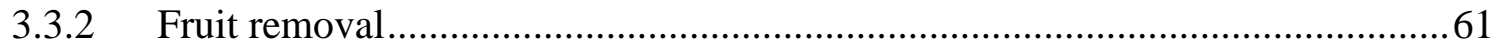

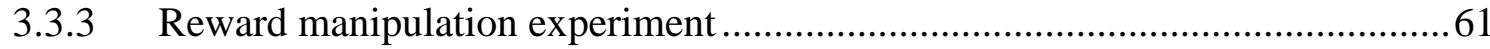

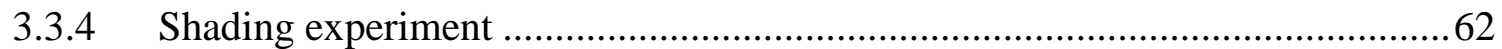

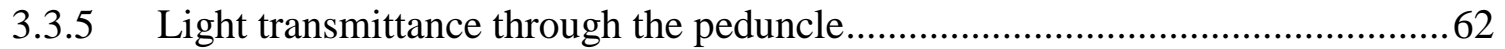

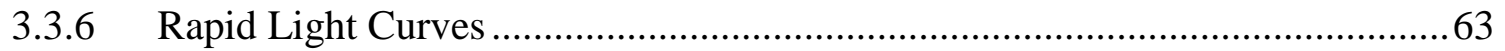

3.3.7 Photoinhibitory treatments for S. nigra peduncles .......................................63

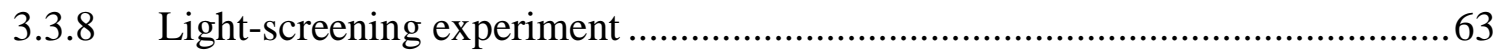

3.3.9 Photoinhibitory treatments for S. nigra petioles ............................................6 64

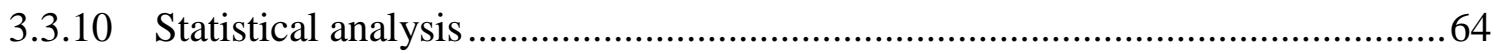

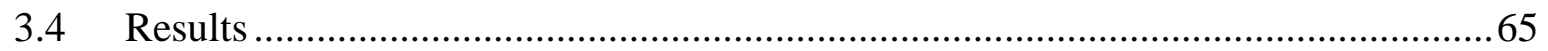

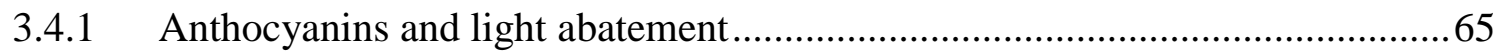

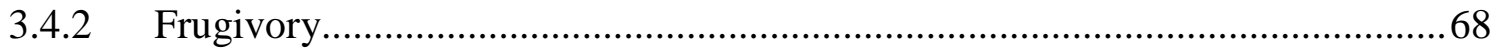

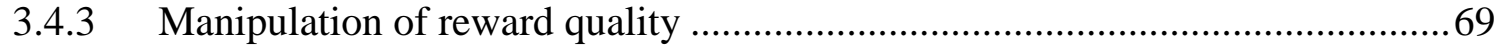

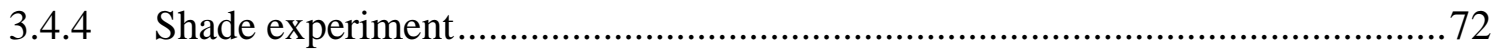


3.4.5 Chlorophyll fluorescence light response curves for S. nigra peduncles 72

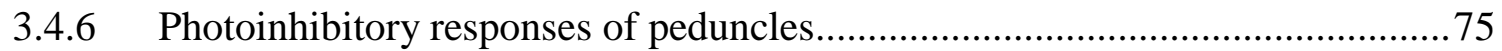

3.4.7 Light response curves and photoinhibitory responses for petioles ....................76

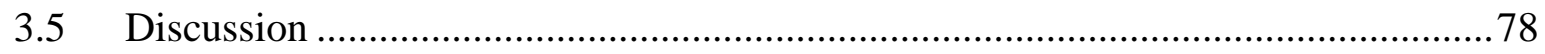

3.5.1 Support for a physiological function for anthocyanins in S. nigra peduncles ... 79

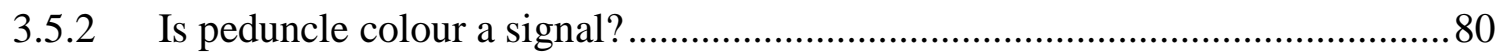

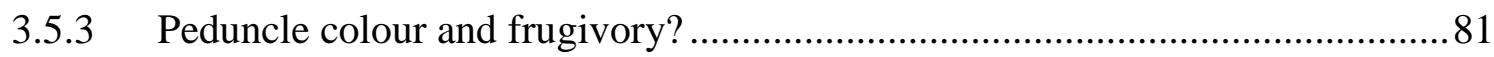

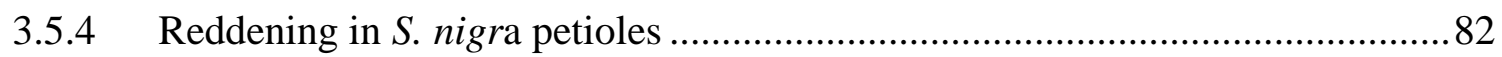

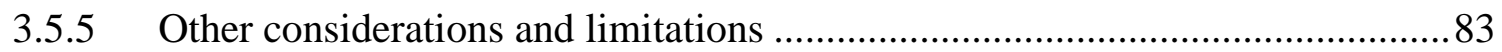

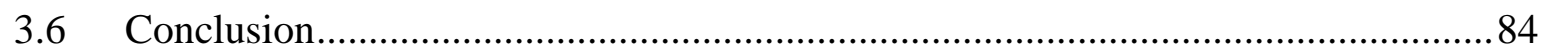

Chapter 4: Do anthocyanins prolong senescence in Sambucus canadensis peduncles? ..........85

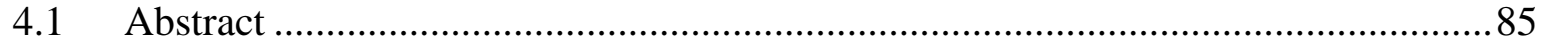

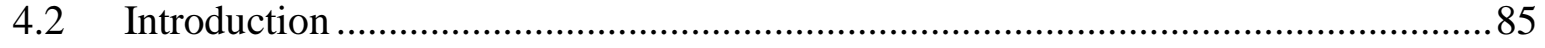

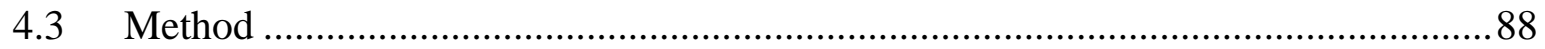

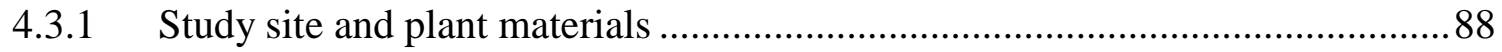

4.3.2 Chlorophyll florescence parameters before, during, and after fruit maturation 88

4.3.3 Anthocyanin, chlorophyll and carotenoid quantification................................ 89

4.3.4 Comparative rates of xanthophyll de-epoxidation ........................................ 89

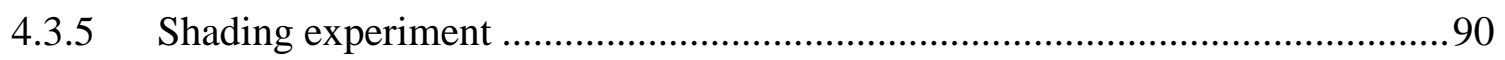

4.3.6 Longevity and nitrogen resorption experiment .............................................. 90

4.3.7 Comparative analysis of tensile strength ..................................................... 91

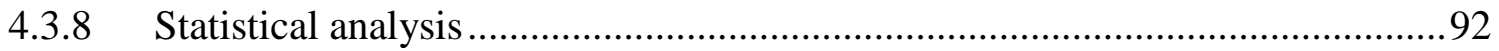

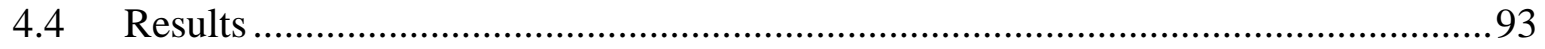

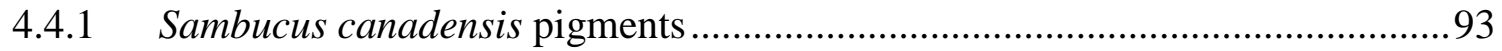

4.4.2 Parameters of photosynthetic health before, during, and after fruit maturation 96

4.4.3 Comparative rates of xanthophyll de-epoxidation .......................................... 98

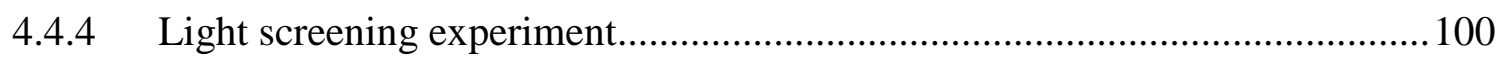




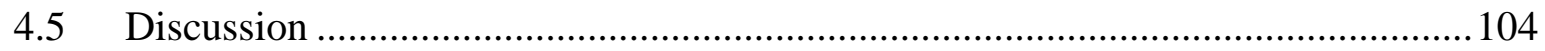

4.5.1 The similarities between red senescing peduncles and red senescing leaves .. 104

4.5.2 Complimentary light screening mechanisms in S. canadensis peduncles ....... 105

4.5.3 Why might anthocyanin accumulation prolong senescence? .......................... 106

4.5.4 Are anthocyanins responsible for a prolonged senescence? .......................... 107

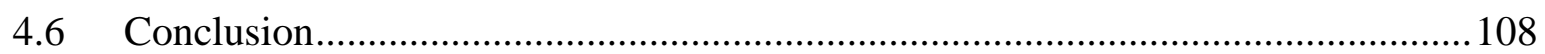

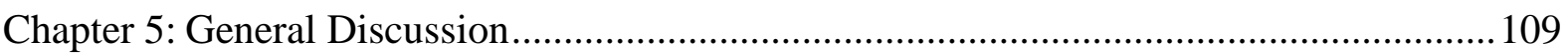

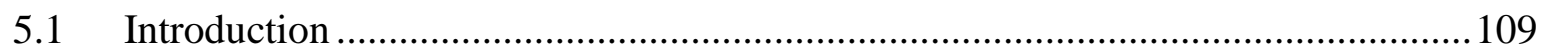

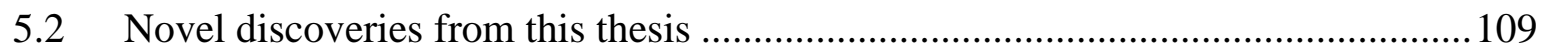

5.3 The significance of identifying a communicative function for red $P$. colorata leaf margins

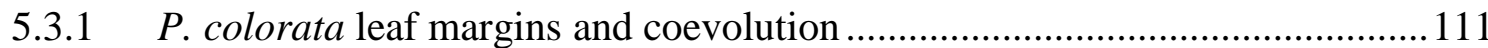

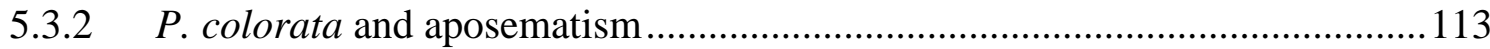

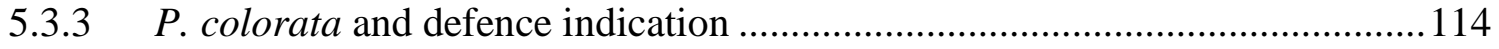

5.3.4 Protection of photo-labile defence compounds................................................ 114

5.4 The significance of identifying a physiological function for red Sambucus spp.

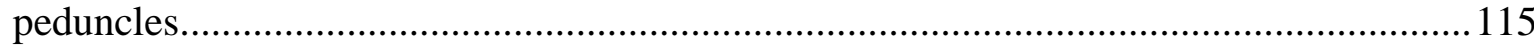

5.4.1 Frugivore attraction vs. photoprotection ................................................... 115

5.4.2 Could anthocyanins prolong senescence in other plant organs? .....................116

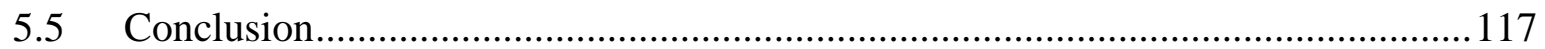

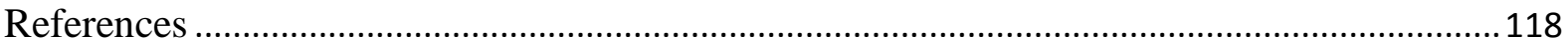




\section{List of Figures}

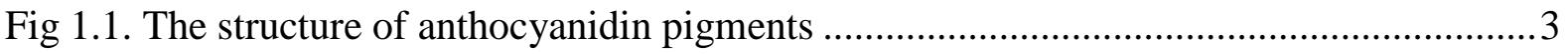

Fig 1.2. Photographs of reddening among vegetative organs. Adventitious roots of Metrosideros excelsa (a); stem of Aristotelia serrata (b); hypocotyl of Myoporum laetum (c); entire leaf lamina of Lophomyrtus bullata (d); leaf margins and areas of herbivory damage on Pseudowintera colorata (e); irregular patches on leaf of Elaeocarpus dentatus (f); senescing foliage of Brachyglottis repanda (g); juvenile foliage of Berberis darwinii (h); petioles of Aristotelia serrata (i)............................5

Fig 1.3. The relative extent of photodamage for different wavelengths of sunlight................8

Fig 1.4. Photographs of red, purple or black peduncles (a-g), rays (b-g), rachides (b, e-g) and pedicels (b-g) among the reproductive structures of Taraxacum officinale (a), Libertia peregrinans (b), Pseudopanax arboreus (c), Sambucus nigra (d), Lunaria annua (e), Cortaderia jubata (f) and assorted grasses (g)

Fig 2.1. Red- (a, c, f) and green- (b, d, g) margined leaves of Pseudowintera colorata: (a, b) photographs of individual branches; (c, d) photographs of leaf laminae; (e) polygodial chemical structure; (f, g) photomicrographs of transverse sections .........34

Fig 2.2. Feeding trial enclosure containing a green- (left) and red- (right) margined Pseudowintera colorata leaf and a Ctenopseustis obliquana larva

Fig 2.3. Oviposition trial enclosure containing a green- (left) and red- (right) margined leaf.40

Fig 2.4. Mean $( \pm$ SE) reflectance values between $400 \mathrm{~nm}$ and $700 \mathrm{~nm}$ for the margins of red(white) and green-margined (grey) Pseudowintera colorata leaves

Fig 2.5. Positional differences in red margin width as percentage of lamina width (a) and area eaten (a) of Pseudowintera colorata leaves

Fig 2.6. Concentrations of polygodial (a) and anthocyanins (a) in the leaf margin and interior of red- (white bars) and green-margined (grey bars) Pseudowintera colorata leaves

Fig 2.7. Area eaten and relative margin width of Pseudowintera colorata leaves from nodes 1 (a), 3 (b) and 7 (c)

Fig 2.8. The distribution of herbivory within Pseudowintera colorata leaves

Fig 2.9. Area of red- (white bars) and green-margined (grey bars) Pseudowintera colorata leaves eaten by Ctenopseustis obliquana larvae under white, green, or red light, or darkness

Fig 3.1. Infructescences of Sambucus nigra. Photograph of red and green peduncles (A), and photomicrographs of transverse sections through red (B) and green (C) peduncles.66

Fig 3.2. Light transmittance through peduncles of Sambucus nigra. (A) Spectral profile of transmitted light at a tissue depth of $40 \mu \mathrm{m}$. Profiles of (B) blue, (C) green and (D) 
red light transmitted at progressive depths through red $(O)$ and green $(0)$ peduncles

Fig 3.3. Photograph of (A) red and (B) green S. nigra petioles and photomicrographs of transverse sections of (C) red and (D) green S. nigra petioles.

Fig 3.4. Fruit sugar concentration (degrees Brix) and ray anthocyanin concentration for Sambucus nigra infructescences.

Fig 3.5. Mean ( \pm SE) soluble sugar for fruit $(A)$, peduncle (B, closed bars) and ray (B, open bars), as well as anthocyanin concentrations in the peduncle (C, closed bars) and ray (C, closed bars) of ripe Sambucus nigra infructescences. Treatments: (Control) all fruits retained; $80 \%$ of fruits removed before ripening; $50 \%$ fruits were removed randomly across infructescence; $50 \%$ of fruits removed from one side of the infructescence .71

Fig 3.6. Mean ( \pm SE) anthocyanin concentrations for Sambucus nigra peduncles and rays that were artificially shaded (closed bars) or exposed to full sunlight (open bars) during development.

Fig 3.7. Rapid light response curves for photochemical quantum yield (ФPSII), photochemical quenching (qP) and non-photochemical quenching (NPQ) for red (०) and green $(\bullet)$ Sambucus nigra peduncles.

Fig 3.8. Decline in quantum yield after (A) $4 \mathrm{~h}$ exposure to $1200 \mu \mathrm{mol} \mathrm{m} \mathrm{s}^{-2}$ white light and $1 \mathrm{~h}$ re-acclimation in the dark, for entirely green (closed bars) and mixed red/ green (open bars), or (B) $6 \mathrm{~h}$ exposure to $500 \mu \mathrm{mol} \mathrm{m} \mathrm{m}^{-2} \mathrm{~s}^{-1}$ blue, green or red light for entirely green (closed) and entirely red (open), Sambucus nigra peduncles .... 75

Fig 3.9. Light response curves for photochemical quantum yield (Fs/Fm'), non-photochemical quenching (NPQ) and photochemical quenching of photosystem II for green and red Sambucus nigra petioles. .77

Fig 3.10. Depression in $\mathrm{Fv} / \mathrm{Fm}$ for red and green petioles after exposure to four hours of $1200 \mu \mathrm{mol} \mathrm{m} \mathrm{m}^{-2}$ white light at $21^{\circ} \mathrm{C}$, and $4{ }^{\circ} \mathrm{C}$. 78

Fig 4.1. Infructescences of Sambucus canadensis. Photograph of infructescences bearing red (A) and green (B) peduncles, and photomicrographs of transverse sections through red $(C)$ and green $(D)$ peduncles.

Fig 4.2. Mean ( \pm SE) anthocyanin (A), chlorophyll (B) and carotenoid (C) concentrations of shaded (closed bars) and exposed (open bars) peduncles. Measurements were taken while fruits were unripe, while fruits were ripening, and after the fruits had been removed

Fig 4.3. Light response curves for photochemical quantum yield $\left(\Phi_{P S I I}\right)$ and nonphotochemical quenching (NPQ) for sun-acclimated $(\bullet)$ and shade-acclimated (०) peduncles before (top), during (middle) and after fruit ripening (below) 98

Fig 4.4. Mean ( \pm SE) ratios of xanthophyll to chlorophyll concentrations (top) and deepoxidized xanthophylls, zeaxanthin and antheraxanthin, to total xanthophyll concentrations (bottom) for red and green Sambucus canadensis peduncles.... 
Fig 4.5. Proportions of green Sambucus canadensis infructescences which abscised after peduncles had been covered with either a red filter (solid line) or transparent light filter (dotted line).

Fig 4.6. Mean $( \pm S E)$ content of thiobarbituric acid-reactive substances (TBARS; above) and nitrogen (below) from green Sambucus canadensis rays and peduncles (respectively), each bearing either a transparent, or a red, anthocyanic light filter. 101

Fig 4.7. Mean $( \pm \mathrm{SE})$ breaking force (top) and breaking extension (bottom) of red and green Sambucus canadensis peduncles 103 


\section{List of tables}

Table 1.1. Putative physiological functions for anthocyanins in vegetative organs................ 16

Table 2.1. Mean ( $\pm \mathrm{SE})$ numbers of Ctenopseustis obliquana eggs oviposited on green- and

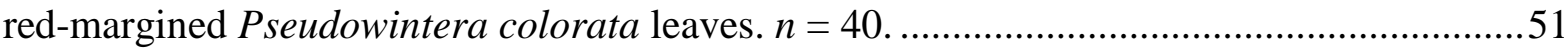

Table 4.1. Mean $( \pm \mathrm{SE})$ pigment concentrations from Sambucus canadensis peduncles that were either exposed, covered by transparent light filter or a neutral density light filter. $n=8$. 


\section{Chapter 1: Redressing the roles of anthocyanins in vegetative and reproductive organs - introduction and literature review}

\section{$1.1 \quad$ Plant pigments and their functions}

The pigments found in plants represent a biochemically diverse group and are responsible for imparting an equally diverse palate of colours. For some of these pigments, we have a thorough understanding of the function they perform and their subsequent significance to a plant's health and vigour. For example, we know that the chlorophylls, the pigments which impart green hues in plants, are responsible for photosynthesis and the conversion of light energy into chemical energy. We also know that the xanthophylls, which belong to the orange and yellow carotenoid family, are responsible for thermal energy dissipation, converting excess light energy to heat. Anthocyanins represent one the most structurally diverse and prevalent families of plant pigments which impart a variety of hues from red to blue to black. Yet the adaptive significance of anthocyanins in plants, in both reproductive and vegetative organs, has been the subject of debate for over a century (Gould, 2010). Originally investigated for their importance in understanding the laws of heredity and genetics (Wheldale, 1916), today anthocyanin research is of considerable economic importance, especially in the fields of viticulture and floriculture (Boulton, 2001; Nishihara \& Nakatsuka, 2011). The biosynthetic pathway, molecular control of its biosynthesis, and the biochemical properties of anthocyanins are now well established (Castañeda-Ovando et al., 2009), as are the nutritional benefits of consuming anthocyanins (Stintzing \& Carle, 2004). It is therefore surprising that the benefits they confer to plants themselves remain a matter of contention. The first review of anthocyanin function in plants was published in 1916, in which the author described a dichotomy in anthocyanin research (Wheldale, 1916). Those studies which had investigated possible physiological functions for anthocyanins had been considered exclusively in vegetative organs. In contrast, the putative communicative significance of anthocyanic colour change had been considered exclusively in flowers and fruits.

In this review, I aim to show that this dichotomy in anthocyanin research still exists, and argue why it inhibits our understanding of anthocyanin function in plants. First (section 1.2), I will discuss the biochemical and spectral properties of anthocyanins, as well as patterns of anthocyanin distribution within plants. Second (section 1.3), I will review the physiological functions proposed for anthocyanins in vegetative organs, and argue that these functions 
might similarly apply to certain reproductive organs. Third (section 1.4), I will review the communicative functions proposed for anthocyanins in vegetative tissues, and discuss the limitations of the current research. Finally (section 1.5), I will present two model scenarios for redressing this dichotomy, the first being a test of a communicative function for anthocyanins in vegetative tissues, the second being a test of a physiological function in reproductive organs.

\subsection{Anthocyanin biochemistry and distribution}

\subsubsection{Biochemical and spectral properties of anthocyanins}

The chemistry and biosynthesis of anthocyanins are well known. Anthocyanins belong to a class of diverse compounds called flavonoids. Derived from the phenolpropanoid pathway, anthocyanins represent the combination of one or more sugar molecules to that of an anthocyanidin aglycone. Anthocyanidins all share the same basic skeleton (the flavylium cation: Fig 1.1), but are defined by the number and position of attached hydroxyl and/or methyl groups (Delgado-Vargas et al., 2000). At least 35 anthocyanidin groups have been identified (Andersen \& Jordheim, 2010); the most common of those, cyanidin, delphinidin and pelargoninodin, are present in $80 \%$ of pigmented leaves, $70 \%$ of fruits and $50 \%$ of flowers (Kong et al., 2003). The classification of an anthocyanin molecule reflects its anthocyanidin, and the position, type and number of sugar molecules attached; to date, about 650 anthocyanins have been identified (Andersen \& Jordheim, 2010). 


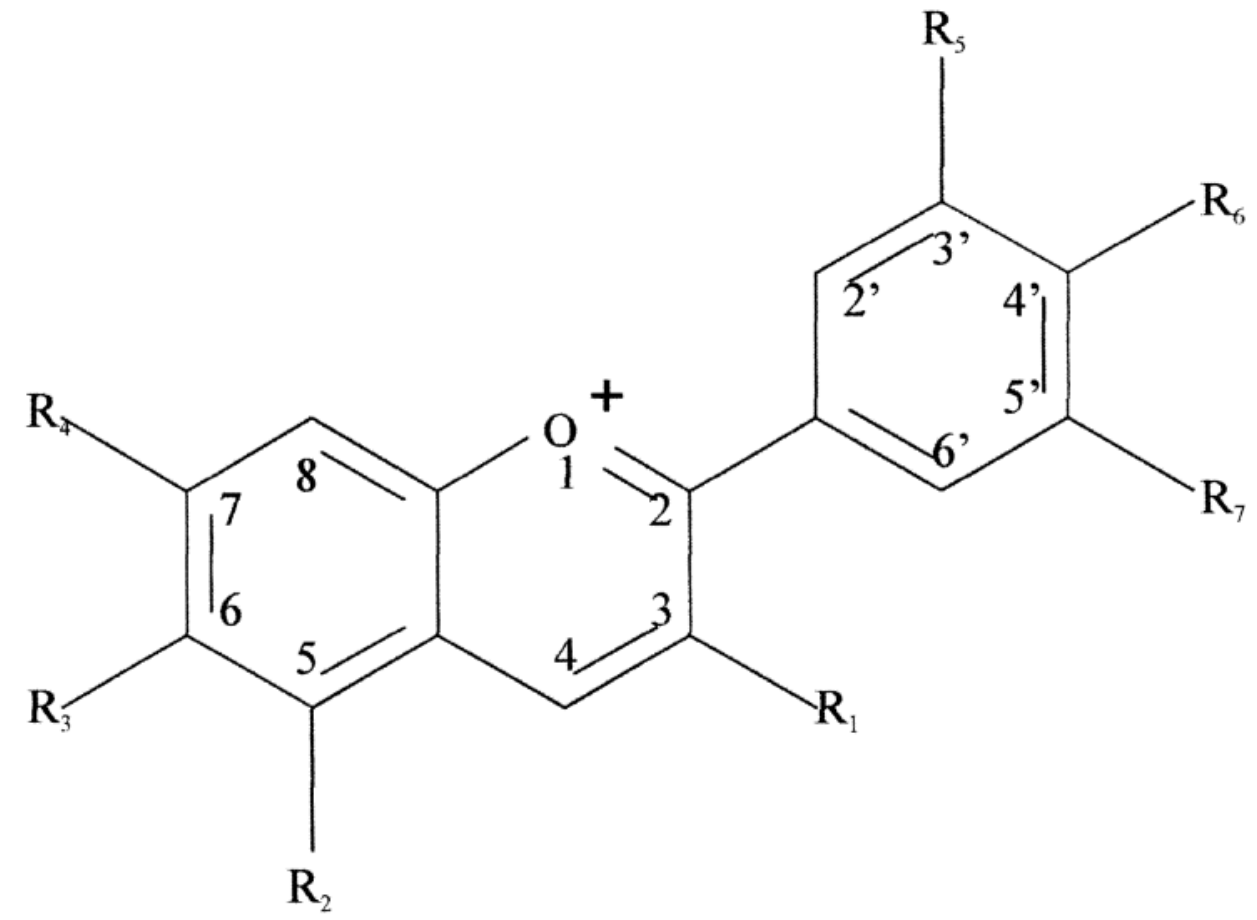

Fig 1.1. The structure of anthocyanidin pigments, the classification of which is determined by substituting R with $\mathrm{H}, \mathrm{OH}$ or $\mathrm{OCH}_{3}$. Source: Delgado-Vargas et al. (2000)

The colour imparted by anthocyanin accumulation is attributable to a number of factors. The structure of the anthocyanidin, the type and extent of glycosylation and acylation, the presence of metal complexes and co-pigmentation all influence the perceived colour of anthocyanins, as do external factors such as vacuolar $\mathrm{pH}$ and temperature (Delgado-Vargas et al., 2000; Kong et al., 2003; Andersen \& Jordheim, 2010). The variety of colours present amongst the reproductive organs of plants is remarkable, especially amongst flowers (Stournaras et al., 2013). Anthocyanins are responsible for imparting hues from red, to blue, to purple, or even black. In contrast, anthocyanins in vegetative organs are most commonly perceived as red or brown, and only rarely appear black (Hatier et al., 2013). From the numerous studies done to determine the optical properties of anthocyanins in leaves, a common absorption spectrum, peaking in the green wavelengths $(500-600 \mathrm{~nm})$, is produced (Neill \& Gould, 2000; Gitelson et al., 2001; Merzlyak et al., 2008; Gould et al., 2010; Zhang et al., 2011). By absorbing green wavelengths, anthocyanins increase the ratio of red: green light that an anthocyanic leaf reflects, and are thus, perceived as red. 


\subsubsection{The histological, morphological and temporal distribution of anthocyanins}

Anthocyanin synthesis is cytoplasmic, most likely occurring on the membrane of the endoplasmic reticulum (Pourcel et al., 2012). Following glycosylation, anthocyanin pigmentation becomes apparent when transported to the low $\mathrm{pH}$ of the vacuole. It is here that anthocyanins are most abundant. The cell types for which anthocyanins have been reported are numerous. They may be in the epidermal and/or sub-epidermal cells, in vascular parenchyma, or present amongst the palisade or spongy mesophyll (Hatier \& Gould, 2009). They may be found in trichomes (Landi et al., 2014), or bundle sheath cells and within a given tissue, may be continuous or sporadically distributed. Anthocyanins are thus, diverse in their histological distribution, perhaps a consequence of multiple selective pressures.

In reproductive organs, anthocyanic colouration is most commonly associated with the conspicuous displays seen in flowers and fruits. However, anthocyanins also cause reddening in rachides, peduncles, pedicels and bracts. In vegetative organs, anthocyanins appear in roots, both aerial (Solangaarachchi \& Gould, 2001) and subterranean, as well as in stolons, rhizomes and tubers (Hatier \& Gould, 2009). Anthocyanic colouration is commonly seen in stems (Gould et al., 2010), hypocotyls, and leaves, and may envelop the entire organ, or portions thereof (Fig 1.2).

The timing of anthocyanin accumulation can also be highly variable between species. In reproductive organs, anthocyanin accumulation typically coincides with fruit or flower maturation, yet for some species of Miconia, anthocyanins are produced during fruit development, and are absent when fruits mature. For vegetative tissues, anthocyanic reddening can be present for the duration of an organ's ontogeny, or may be restricted to its juvenile (Dominy et al., 2002) or senescent stages (Lee, 2002). The foliar accumulation of anthocyanins, displayed by deciduous species during autumn, would be the most recognizable example of this. For some plants, anthocyanin accumulation is a response to mechanical wounding, such as that exerted by herbivores (Gould et al., 2002a), while in others, anthocyanins are produced in response to environmental stressors such as light, cold, salt, heavy metal and drought (Chalker-Scott, 1999). The expression of anthocyanic colouration is evidently a diverse phenomenon within the plant kingdom, displaying an enormous amount of histological, spatial and temporal variability. Therefore, it is not surprising that the hypotheses proposed to explain the adaptive significance of anthocyanins are similarly numerous. 


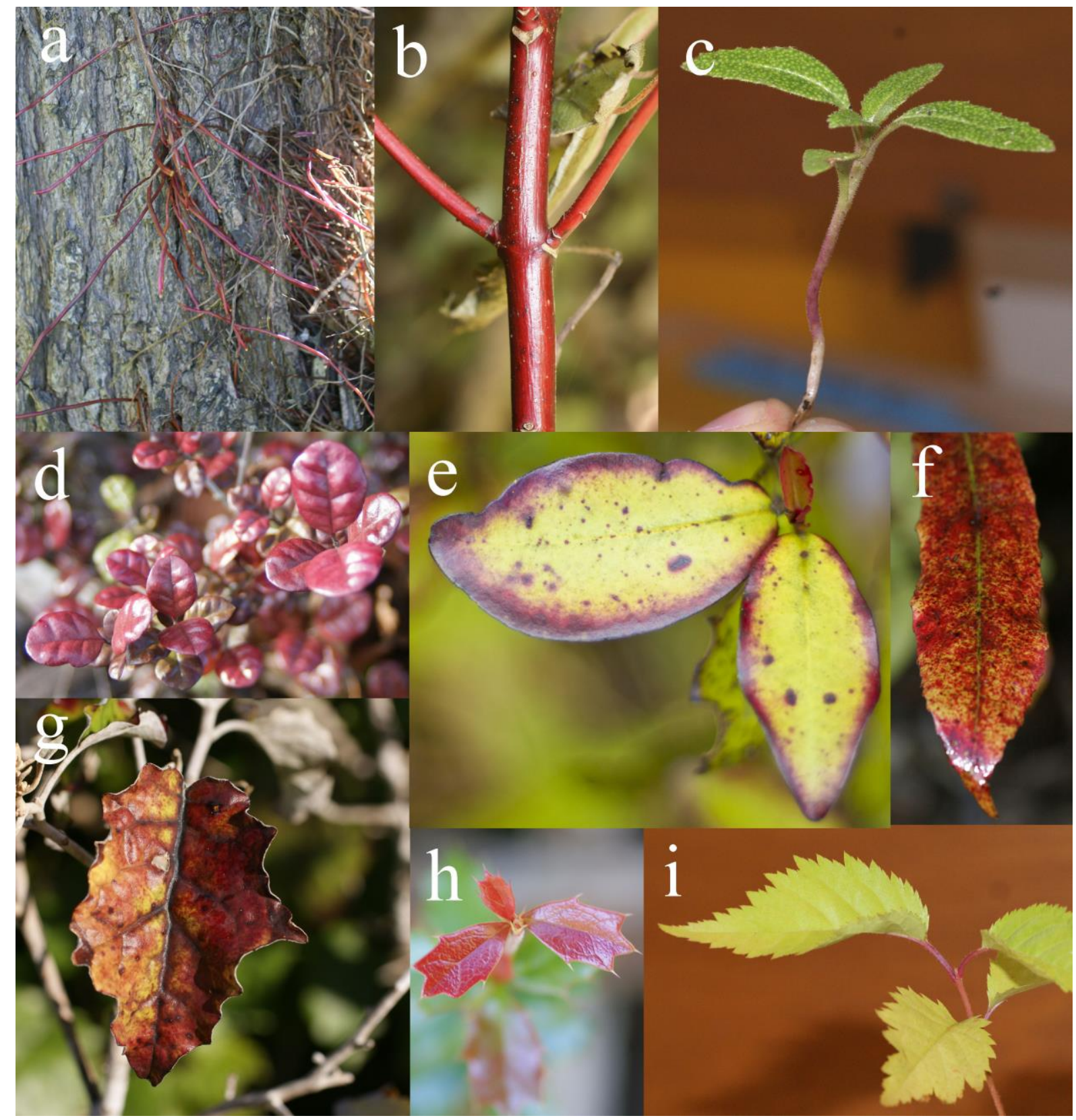

Fig 1.2. Photographs of reddening among vegetative organs. Adventitious roots of Metrosideros excelsa (a); stem of Aristotelia serrata (b); hypocotyl of Myoporum laetum (c); entire leaf lamina of Lophomyrtus bullata (d); leaf margins and sites of herbivore damage on Pseudowintera colorata (e); irregular patches on a Elaeocarpus dentatus leaf (f); senescing foliage of Brachyglottis repanda (g); juvenile foliage of Berberis darwinii (h); petioles of Aristotelia serrata (i).

\subsection{Physiological significance of anthocyanin accumulation}

Anthocyanic colouration may be costly for plants. From anthocyanidin synthesis, to glycosylation and finally transport to the cell vacuole, anthocyanin production involves a 
series of ATP driven, metabolic processes (Alfenito et al., 1998; Gould, 2004). The significance of this production cost has been questioned, and Foyer et al. (2007) argued that "anthocyanins [...] represent a small fraction of the photoassimilate produced in a single day". However, for photosynthetic organs, anthocyanins may inflict an additional cost. By absorbing those wavelengths that would normally contribute towards photosynthesis in the lower mesophyll tissues, anthocyanins may reduce carbon assimilation, and possibly carbohydrate production (Hatier et al., 2013). This cost, combined with the knowledge that patterns of anthocyanic colouration have evolved multiple times in both vegetative (Archetti, 2009c), and reproductive organs (Stournaras et al., 2013), implies that anthocyanins impart a selective advantage. A number of physiological functions have been proposed and tested, which aim to identify what that selective advantage might be. However, the vast majority of these physiological functions have been studied exclusively in vegetative organs. The following section will review those functions which have received the most attention, and subsequently discuss their applicability to reproductive tissues.

\subsubsection{Photoinhibition and its prevention by anthocyanins}

During photosynthesis, photosystem II is subject to a constant cycle of damage and repair. Photodamage of the PSII reaction centre begins with inactivation of the oxygen evolving complex (OEC) (Hakala et al., 2006; Tyystjärvi, 2008; Takahashi \& Badger, 2011). The OEC is responsible for oxidation of water molecules (Padhye et al., 1986; Yachandra et al., 1996), the disruption of which by UV and visible wavelengths inhibits electron donation to the PSII reaction centre. While the mechanism by which photodamage via OEC inactivation remains unresolved, the absence of this electron donation inhibits reduction of the PSII primary donor (P680), and it is believed that P680 in an excited/oxidized state damages the PSII reaction centre (Takahashi \& Murata, 2008). Photodamage is unavoidable (Hakala et al., 2006; Nishiyama et al., 2006; Tyystjärvi, 2008), and its severity is directly proportional to incident light intensity (Jones \& Kok, 1966; Tyystjärvi \& Aro, 1996; Lee et al., 2001; Hendrickson et $a l .$, 2005). This means that PSII repair is a continuous process during photosynthesis. It is achieved by proteolysis of the D1 protein, and subsequent D1 synthesis followed by insertion back into the PSII reaction centre. Photoinactivation refers to the condition experienced when the rate of photo-damage exceeds the rate of repair, and is characterised by a decline in photosynthetic efficiency. This decline reduces carbon fixation, of which one of the products, glycerate-3-phosphate, is critical for D1 synthesis (Takahashi \& Murata, 2005; Takahashi \& 
Murata, 2006). Prolonged exposure to excess light thereby reduces D1 synthesis, the very protein responsible for repair of the PSII reaction centre. Prolonged exposure therefore induces photoinhibition, the condition where both the PSII reaction centre and its repair mechanism are damaged, and is characterised by a long term depression in quantum yield following light stress.

Photodamage peaks when subjected to UV wavelengths between $300 \mathrm{~nm}$ and $350 \mathrm{~nm}$, as well as visible wavelengths between $500 \mathrm{~nm}$ and $700 \mathrm{~nm}$ (Takahashi et al., 2010). Sunlight contains UV at relatively low intensities compared to visible light (Fig 1.3), thus, those green/red wavelengths between $500 \mathrm{~nm}$ and $700 \mathrm{~nm}$ are the most significant contributors to photodamage. The absorption spectrum for anthocyanins, reported for leaves and stems, peaks in green light (Neill \& Gould, 2000; Gitelson et al., 2001; Merzlyak et al., 2008; Gould et al., 2010). By screening photosynthetic tissues from a proportion of those wavelengths responsible for photodamage, anthocyanins are believed to ameliorate photoinhibition. Termed the 'light-screening' or 'photoprotection' hypothesis, numerous studies have reported a reduction in the severity of photoinhibition for red leaves and stems, compared to green, when exposed to excess light (Gould et al., 1995; Dodd et al., 1998; Feild et al., 2001; Manetas et al., 2002; Steyn et al., 2002; Gould, 2004; Hughes et al., 2005; Shao et al., 2008; Hatier \& Gould, 2009; Gould et al., 2010; Zhang et al., 2010; Nielsen \& Simonsen, 2011; Zhang et al., 2011; Hughes et al., 2014; Landi et al., 2014; Tanino et al.2014; Tattini et al., 2014), although exceptions to this have been noted (See section 1.3.2). Particularly compelling evidence for the role of anthocyanins in avoiding light stress comes from those studies which compare rates of photoinhibition under monochromatic light treatments. Plants employ a number of strategies to avoid light stress and so identifying the contribution anthocyanins make can often be challenging. Nonetheless, through the judicious use of monochromatic red, green or blue light, several studies have shown that a reduction in photoinhibition, for red leaves compared to green, is either specific to, or more dramatic when, tissues are irradiated with green wavelengths which anthocyanins absorb (Feild et al., 2001; Gould et al., 2002b; Landi et al., 2014). 


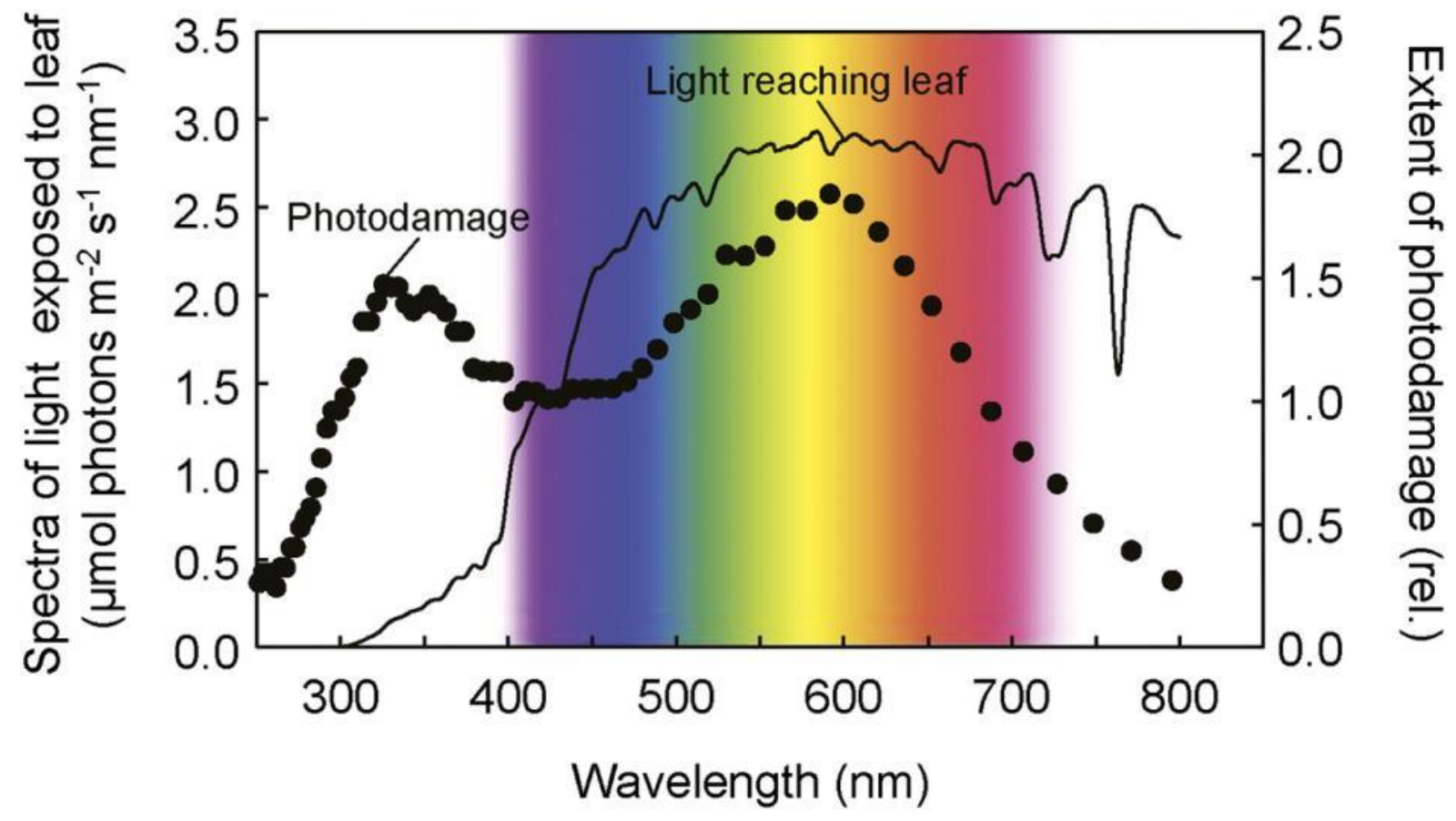

Fig 1.3. The relative extent of photodamage for different wavelengths of sunlight. Source: Takahashi et al. (2010).

\subsubsection{Criticisms of the photoprotective hypothesis}

The photoprotective hypothesis has been criticised because anthocyanins absorb wavelengths that are least utilised by chloroplasts (Manetas, 2006; Archetti et al., 2009; Juvany et al., 2013). However, we now know that the relative contribution that different wavelengths make to photodamage is unrelated to the spectrum of chlorophyll absorption (Fig 1.3) and that red and green wavelengths are those that are particularly detrimental. In this way, anthocyanin absorption appears especially suited to maximising absorption of those visible wavelengths which induce photodamage, except when those wavelengths are required for photosynthesis. For this reason, criticism of the photoprotective hypothesis based on the distinct absorption spectra of anthocyanins and chlorophyll is inapplicable.

The photoprotective hypothesis has also drawn criticism (Archetti et al., 2009) because a number of studies appear to contradict its primary prediction, that the addition of anthocyanins will mitigate the severity of photoinhibition. Various experimental studies, comparing photoinhibition for red and green plants, demonstrated either no advantage, or a disadvantage to being red (Williams et al., 2003; Kyparissis et al., 2007; Esteban et al., 2008; Zeliou et al., 2009; Nikiforou \& Manetas, 2010; Manetas \& Buschmann, 2011; Júnior et al., 
2012; Liakopoulos \& Spanorigas, 2012), and Archetti et al. (2009) called for "a reassessment of these contrasting results".

A closer inspection of these studies shows that for many, the experimental design did not isolate the effect of anthocyanins and failed to control for differences in anatomy or physiology when comparing red species (or red phenotypes within the same species) against green (Burger \& Edwards, 1996; Williams et al., 2003; Kyparissis et al., 2007; Zeliou et al., 2009; Nikiforou \& Manetas, 2010; Manetas \& Buschmann, 2011; Júnior et al., 2012). For example, Kyparissis et al. (2007) compared photoinhibition for green Prunus domestica leaves to that of red Prunus cerasifera var. atropurpurea leaves after both varieties had been grafted onto $P$. domestica rootstocks. This study failed to control for both anatomical and physiological differences between the two species, but most importantly, did not control for the effect of grafting one species onto the rootstock of another. It may be that anthocyanin accumulation contributes towards photoprotection in red leaves, while green leaves receive photoprotection from a variety of alternative mechanisms (Williams et al., 2003), such as an augmented heat dissipation via the xanthophyll cycle (Bilger \& Björkman, 1990). Isolating the specific contribution that anthocyanins make to photoprotection is therefore required. Other studies have been cited with insufficient clarification of the results. For example, Manetas et al. (2003) and Karageorgou \& Manetas (2006) showed that red juvenile leaves of Quercus coccifera, displayed significantly higher photosynthetic efficiencies compared to green leaves while exposed to white light, and were less photoinhibited following light stress. Karageorgou \& Manatas (2006) additionally showed that while the difference in photosynthetic efficiency between red and green phenotypes was small, it disappeared under red light, wavelengths which anthocyanins do not absorb. These two studies collectively showed that red leaves of $Q$. coccifera are less susceptible than green leaves to light stress, and that anthocyanin accumulation is likely to be responsible for this difference. Nonetheless, both of these studies have been cited, even by the authors themselves, as examples where anthocyanins do not provide a photoprotective benefit (Karageorgou et al., 2008; Archetti et al., 2009). Examples such as these risk erroneously inflating the evidence against the photoprotective hypothesis.

Arguably, the strongest criticism to the photoprotection hypothesis is that no study has yet demonstrated that reducing photoinhibition via anthocyanin accumulation provides a fitness benefit. This hypothesis demands that a requirement for photoprotection be a selective force driving anthocyanin accumulation in photosynthetic tissues. Instead, anthocyanins may be 
produced for some other reason, and although they demonstrably reduce photoinhibition (see section 1.3.1), this may not necessarily provide the plant with any long-term fitness gain. To date, only one study has tested whether light-screening via anthocyanin accumulation provides any long term benefit (Hatier et al., 2013). For Ophiopogon planiscapus 'Nigrescens', plants produce leaves that are either green, or when anthocyanic, black. Compared to black leaves, green leaves of $O$. planiscapus displayed lower rates of photosynthetic efficiency when exposed to irradiance levels between 250 and $2000 \mu \mathrm{mol}$ photons $\mathrm{m}^{-2} \mathrm{~s}^{-1}$, and following light and cold stress, displayed a lower recovery from photoinactivation, symptomatic of photoinhibition. In spite of this, green leaves displayed higher rates of $\mathrm{CO}_{2}$ assimilation than did black leaves, and when grown in full sunlight, green leaves branched more abundantly and accumulated shoot biomass more quickly. Although this study did not compare the fitness of the two phenotypes, it did provide a compelling example of where anthocyanins provided a light screening benefit, but that benefit did not lead to long-term success in any measurable way. Perhaps anthocyanins provide a long-term benefit when produced in environmental conditions other than those used in this study, but as yet, this requirement of the photoprotection hypothesis has not been satisfied.

\subsubsection{Anthocyanins and oxidative stress}

Reactive oxygen species (ROS) are an integral component of plant cell metabolism. They act as signalling molecules to initiate processes involved in stress response (Apel \& Hirt, 2004; Triantaphylidès et al., 2008; Møller \& Sweetlove, 2010), and are responsible for oxidising pigments, proteins and lipids during senescence (Hörtensteiner \& Feller, 2002). However, ROS can also be detrimental, and in excess, will impair photosynthetic health (Nishiyama et $a l ., 2001$ ), and even induce premature cell death (Juvany et al., 2013). Singlet oxygen $\left({ }^{1} \mathrm{O}_{2}\right)$ is especially harmful, and represents one of the major ROS in photo-oxidative stress (Triantaphylidès et al., 2008). Singlet oxygen is produced via excitation of the ground triplet state of oxygen $\left({ }^{3} \mathrm{O}_{2}\right)$ by ${ }^{3} \mathrm{P} 680^{+}$, the triplet state of the PSII reaction centre (Krieger-Liszkay, 2005; Asada, 2006), which is in turn produced when excited in the absence of OEC (oxygen evolving complex) electron donation. As the OEC is the likely site of photodamage (see section 1.3.1), photo-oxidation is a direct consequence of photoinactivation. Anthocyanins may reduce singlet oxygen production and photo-oxidative stress by acting as light screens, first by preventing inactivation of the manganese cluster and secondly by reducing excitation pressure on the PSII reaction centre itself (Steyn et al., 2002). However, the extent to which 
anthocyanins reduce singlet oxygen production has yet to be resolved, and few empirical tests exist. Some studies demonstrate a reduction in ROS production for anthocyanic leaves, compared to acyanic leaves (Peng et al., 2006; Zhang et al., 2012), but attributing this difference to the light screening potential of anthocyanins has been questioned (Zhang et al., 2012).

It may be that anthocyanins reduce oxidative damage, not by preventing photo-oxidation, but by scavenging ROS that are produced subsequent to photo-oxidation. It has been demonstrated that anthocyanins are potent antioxidants, displaying up to four times the ROS scavenging potential of trolox (Wang et al., 1997), an industry standard in gauging antioxidant potential. Moreover, in vivo monitoring of an oxidative burst (following mechanical wounding), showed that $\mathrm{H}_{2} \mathrm{O}_{2}$ decreased more rapidly in red (anthocyanic) Pseudowintera colorata leaves than green. While the vacuolar storage of anthocyanins argues against them acting as direct scavengers of ROS produced in the chloroplast, it may be that cytoplasmic anthocyanins are responsible for acting as antioxidants. For example, Zhang et al. (2012) showed that leaves of an acyanic Arabidopsis thaliana mutant subjected to a high irradiance displayed a reduced DPPH scavenging potential and increased oxidative damage (estimated by cell membrane permeability) compared to wild-type anthocyanic leaves, even though anthocyanins were present in concentrations too low to be an effective light screen. They argued that the increased scavenging capacity displayed by wild-type leaves related to an increase in the content of precursors for anthocyanin synthesis (Zhang et al., 2012). Moreover, they showed that the wild-type variety maintained higher quantum efficiencies under high irradiance, and following a light stress treatment, was less photo-inactivated compared to the acyanic mutant. Evidently, anthocyanins are capable of reducing light stress, even when produced in concentrations too low to act as a light screen.

\subsubsection{Anthocyanin accumulation in juvenile leaves}

Photosynthetic tissues are particularly susceptible to light stress during development when chloroplasts are still immature (Juvany et al., 2013), and numerous studies report a reduction in the quantum efficiency of PSII ( $P P S I I)$ for juvenile, compared to mature leaves (Jiang et al., 2005; Maayan et al., 2008; Lepeduš et al., 2011). This reduced efficiency may be a result of increased susceptibility to photodamage (Juvany et al., 2013), or a reduced capacity for repair of the D1 protein (Maayan et al., 2008; Lepeduš et al., 2011). Regardless, this increased susceptibility to light stress implies that chloroplasts of juvenile leaves have a 
greater requirement for photoprotection than do chloroplasts of mature leaves. For many plants, notably tropical species, leaf development coincides with foliar reddening and anthocyanins are thought offer a light screen, reducing photodamage during this time (Dominy et al., 2002). In support of this, numerous studies report either a reduction in the extent of photoinhibition, or an improved photosynthetic efficiency for red juvenile leaves, compared to green mature leaves (Manetas et al., 2002; Manetas et al., 2003; Karageorgou \& Manetas, 2006; Liakopoulos et al., 2006; Hughes et al., 2007; Fondom et al., 2009; Lan et al., 2011; Solovchenko \& Chivkunova, 2011; Ranjan et al., 2014), although the benefits may be small (Karageorgou \& Manetas, 2006).

\subsubsection{Anthocyanin accumulation in senescing leaves}

Senescence represents the final stage of an organ's ontogeny. During this period, proteins, membranes, lipids and most importantly chloroplasts, are catabolised (Guo \& Gan, 2005; Lim et al., 2007), the products of which are then exported to other organs in the plant. Termed nutrient recycling, this process occurs in roots, shoots and leaves (Freschet et al., 2010), and by preventing a loss of nutrients, is seen as a significant contributor to plant fitness (May \& Killingbeck, 1992). Chloroplasts can contain up to $70 \%$ of a photosynthetic organ's nitrogen content (Lim et al., 2007), thus, are especially significant contributors to nutrient recycling. However, nitrogen recycling is potentially impaired by light stress (Feild et al., 2001), and photosynthetic tissues are particularly susceptible to photoinhibition during this period (Wingler et al., 2006; Juvany et al., 2013). By performing a photoprotective role in senescing leaves, anthocyanins are thought to mitigate the severity of light stress, thereby enhancing nutrient recycling in senescing leaves (Feild et al., 2001; Hoch et al., 2001).

This 'nitrogen resorption' hypothesis has received considerable support from several studies. For Acer saccharum, the timing and extent of anthocyanin accumulation correlates negatively with foliar nitrogen content (Schaberg et al., 2003); those individuals that are deficient in nitrogen, and thus possess the highest demand for nutrient recycling, turned red earlier and more intensely. In Cornus stolonifera, anthocyanin accumulation is exclusive to leaves senescing in full sunlight, and compared to shade-acclimated yellow-senescing leaves, red leaves are less photoinhibited following light stress (Feild et al., 2001). This same study also found that nitrogen resorption in red leaves was comparable to that of yellow leaves, even though red leaves senesce in a high-light environment. Even more convincing is the work by 
Hoch et al (2003), who compared the photosynthetic health and nutrient resorption of anthocyanic wild-type individuals from three species, Cornus sericea, Vaccinium elliottii, and Viburnum sargentii against acyanic mutants of those same species. For each species, the red wild-type varieties maintained higher photosynthetic efficiency, as estimated by chlorophyll fluorescence, under light and cold stress and recovered more quickly following the treatment period. Furthermore, the red wild-type varieties displayed higher rates of nitrogen resorption compared to the acyanic mutants, but only when senescence took place under high light and cold stress; when both varieties senesced in a lower light, warmer environment, nitrogen resorption was comparable. These results suggest that anthocyanin accumulation does not necessarily enhance nitrogen resorption, but rather, prevents the reduction in nitrogen resorption that senescing leaves experience under light stress.

\subsubsection{Criticism of the nitrogen resorption hypothesis}

Manetas \& Buschmann (2011) provided an example which purportedly (Juvany et al., 2013) contradicted a requirement of the nitrogen resorption hypothesis, namely that anthocyanins reduce light stress in senescing leaves. For two species bearing senescing leaves of varying redness, no relationship was found between anthocyanin accumulation and any proxy of photosynthetic performance ie, anthocyanin concentrations did not correlate to maximum quantum yield of photosystem II, quantum efficiency of photosystem II or severity of photoinhibition following light stress. Like those studies discussed in section 1.3.2, this experimental design failed to isolate the effect of anthocyanins on reducing light stress, and in particular, failed to control for possible variability in senescence's progression among the leaves measured. This is important because senescence coincides with an increase in anthocyanins, a decrease in chlorophyll and an increased susceptibility to light stress (see section 1.3.5). If variability in the progression of senescence is present among the leaves measured, Manetas and Buschman (2011) would simply have been comparing green leaves at the early stages of senescence against red leaves at the latter stages of senescence. Consistent with this, the authors also found a negative correlation between anthocyanin concentrations and chlorophyll content. Variability can exist for any number of reasons; it may be that within an individual, leaves initiate senescence at different times, or it may be that the rate at which senescence progresses differs between leaves. Regardless, if we recognise that senescence coincides with a decrease in chlorophyll and an increase in anthocyanin content, Manetas and Buschman's (2011) study instead shows that anthocyanic leaves in the later 
stages of senescence are able to maintain photosynthetic health, comparable to those leaves in the early stages of senescence. This study emphasises the need to isolate the effect of anthocyanins on photosynthetic health, especially when interpreting a null result.

In another study, Misyura et al. (2012) compared the progression of senescence for six acyanic Arabidopsis thaliana mutant varieties, against two anthocyanic wild-type varieties, when all were grown under high-light and nitrogen deficient conditions. In contrast to the nitrogen resorption hypothesis, the initiation of senescence, the rate at which chlorophyll catabolises and the expression of three genes associated with senescence did not differ uniformly between anthocyanic and acyanic varieties. Similarly, plant growth (i.e. biomass) prior to senescence, differed among the eight varieties, but for some of the acyanic mutant strains, biomass was less than that of two wild-type varieties, and for other strains, biomass was more than that of the wild-types. The authors therefore concluded that anthocyanins did not affect plant growth or the progression of senescence under the chronic stress conditions tested. However, there is little indication that the conditions employed in that study were sufficiently detrimental to the quantum efficiency of PSII. The authors assumed that anthocyanin production indicated light stress but at $400 \mu \mathrm{mol}$ photons $\mathrm{m}^{-2} \mathrm{~s}^{-1}$, the intensity of the high light treatment was less than a quarter that of full sunlight. Moreover, five of the six strains displayed significantly higher carotenoid concentrations than the two wild type varieties, potentially indicative of an increase in xanthophyll production. It could be that anthocyanin deficient strains acclimated to the 'high-stress' conditions by investing in an alternative light screening mechanism. If the acyanic varieties acclimated to the high-stress treatment, we might not expect the progression of senescence to differ. Once again, the effect of anthocyanins was not isolated in this study.

\subsubsection{Does anthocyanin accumulation prolong senescence?}

A slight modification of the 'nitrogen resorption' hypothesis was identified by Schaberg et al. (2008), who suggested that anthocyanin accumulation prolongs senescence which enables more time for nutrient recycling. While the mechanism by which this is achieved has yet to be identified, the light screening properties of anthocyanins have been implicated. Ethylene and auxin are among the hormones responsible for coordinating senescence, with ethylene accelerating and auxin retarding the process (Roberts et al., 2000; Roberts et al., 2002; Schaberg et al., 2008). Oxidative stress appears to hasten leaf senescence by increasing a tissue's sensitivity to ethylene while simultaneously reducing auxin transport (Michaeli et al., 
1999a; Michaeli et al., 1999b). By preventing photoinhibition and subsequent ROS production (Schaberg et al., 2008), anthocyanins may be responsible for prolonging leaf senescence thereby enhancing nitrogen resorption. Although few studies have investigated this hypothesis, red senescing species do demonstrate a late leaf abscission, maintain carbon assimilation for longer, and display higher rates of nitrogen resorption, compared to cooccurring acyanic species (Zhang et al., 2013b) as well as possess several physical characteristics indicative of a prolonged senescence (Schaberg et al., 2008). However, it is yet to be shown that a prolonged senescence for red leaves is directly attributable to the presence of anthocyanins.

\subsubsection{Other putative physiological roles for anthocyanins}

A number of additional physiological functions have been proposed for anthocyanins in vegetative organs but are of little relevance here. Table 1.1 describes these additional hypotheses and provides the literature relevant to each. 
Table 1.1. Putative physiological functions for anthocyanins in vegetative organs.

\begin{tabular}{|c|c|c|}
\hline Function & Description & Pertinent references \\
\hline $\begin{array}{l}\text { Drought/ salinity } \\
\text { tolerance }\end{array}$ & $\begin{array}{l}\text { Anthocyanins act as osmoregulators or } \\
\text { reduce drought/ salt induced oxidative } \\
\text { stress. }\end{array}$ & $\begin{array}{l}\text { Salt stress } \\
\text { Kaliamoorthy \& Rao, 1995; Chalker-Scott, } \\
\text { 1999; Chalker-Scott, 2002; Ery1lmaz, 2006; } \\
\text { Chaves } \text { et al., 2009; Dkhil \& Denden, 2012; } \\
\text { Cheng et al., 2013; Garriga et al., 2014; } \\
\text { Rasheed } \text { et al., 2014 } \\
\text { Drought stress } \\
\text { Balakumar et al., 1993; Sherwin \& Farrant, } \\
\text { 1998; Nogués \& Baker, 2000; Alexieva et } \\
\text { al., 2001; Tahkokorpi et al., 2007; Efeoğlu } \\
\text { et al., 2009; Hughes et al., 2010a; Sperdouli } \\
\text { \& Moustakas, 2012; Hughes et al., } 2013\end{array}$ \\
\hline Cold tolerance & $\begin{array}{l}\text { Low temperatures increase a leaf's } \\
\text { susceptibility to photo-oxidative stress. } \\
\text { Anthocyanins reduce this by acting as } \\
\text { light screens or antioxidants. } \\
\text { Alternatively, anthocyanins raise leaf } \\
\text { temperature by converting light energy } \\
\text { into heat. }\end{array}$ & $\begin{array}{l}\text { Smith, 1909; Shichijo et al., 1993; Christie } \\
\text { et al., 1994; Chalker-Scott, 1999; Solecka } \text { et } \\
\text { al., 1999; Pietrini et al., 2002; Hughes } \text { et al., } \\
\text { 2005; Tahkokorpi } \text { et al., 2007; Ruelland } \text { et } \\
\text { al., 2009; Wang et al., 2009; Hughes } \text { et al., } \\
\text { 2010a; Løvdal et al., 2010; Catalá et al., } \\
\text { 2011; Hughes, 2011; Dufoo-Hurtado } \text { et al., } \\
\text { 2013 }\end{array}$ \\
\hline $\begin{array}{l}\text { Heavy metal } \\
\text { tolerance }\end{array}$ & $\begin{array}{l}\text { Metals are bound to anthocyanins via } \\
\text { chelation then sequestered to the } \\
\text { vacuole. Alternatively, anthocyanins } \\
\text { reduce oxidative stress induced by heavy } \\
\text { metals and metalloids. }\end{array}$ & $\begin{array}{l}\text { Dell \& Malajczuk, 1994; Baranowska } \text { et al., } \\
\text { 1996; Hale et al., 2001; Hale et al., 2002; } \\
\text { Nissim-Levi et al., 2003; Gratão et al., 2005; } \\
\text { Glińska et al., 2007; Arreola et al., 2008; } \\
\text { Posmyk et al., 2009; Landi et al., 2012; } \\
\text { Landi et al., 2013; Landi et al., 2014; Tattini } \\
\text { et al., } 2014\end{array}$ \\
\hline UV protection & $\begin{array}{l}\text { Anthocyanins absorb UV radiation, } \\
\text { screening tissues from UV damage }\end{array}$ & $\begin{array}{l}\text { Beggs \& Wellmann, 1985; Takahashi et al., } \\
\text { 1991; Burger \& Edwards, 1996; Woodall \& } \\
\text { Stewart, 1998; Mendez et al., 1999; } \\
\text { Alexieva et al., 2001; Hada et al., 2003; } \\
\text { Newsham et al., 2005; Lau et al., 2006; } \\
\text { Pfündel et al., 2007; Wang et al., 2012; } \\
\text { Tsurunaga et al., 2013; Costa } \text { et al., } 2014\end{array}$ \\
\hline $\begin{array}{l}\text { Back-scatter } \\
\text { hypothesis }\end{array}$ & $\begin{array}{l}\text { Abaxial anthocyanins maximise light } \\
\text { capture in leaves by reflecting red light } \\
\text { back to the mesophyll tissues. }\end{array}$ & $\begin{array}{l}\text { Lee } \text { et al., 1979; Gould } \text { et al., 1995; Hughes } \\
\text { et al., } 2008\end{array}$ \\
\hline
\end{tabular}

\subsubsection{Physiological function in reproductive organs}

There is a long-held consensus that anthocyanin accumulation in flowers is primarily a means of pollinator attraction (Allen, 1879; Schaefer \& Ruxton, 2011). However, the equivalent assertion when applied to fruits and their seed dispersers is contentious (Schaefer \& Schaefer, 2007; Schaefer et al., 2007). A role in frugivore attraction has long been posited for fruits (Allen, 1879; Ridley, 1930), yet anthocyanins might additionally perform essential 
physiological functions in these organs. For many plants, anthocyanins in the fruit are produced in quantities beyond that required for maximal conspicuousness (Schaefer et al., 2008; Schaefer, 2011), possibly indicating a selective pressure beyond that exerted by frugivores. Instead, anthocyanin production corresponds to the metabolic cost (nutrient content) of a fruit (Schaefer et al., 2008). This observation makes sense if the physiological functions already described for anthocyanins in leaves are similarly present in fruits. Accordingly, those plants that invest the most resources into fruit quality are those that invest the most into protecting that fruit from environmental stress. Like leaves, fruits are photosynthetic and are similarly prone to photoinhibition (Steyn, 2009). Ripening often coincides with the breakdown of chlorophyll in fruits, and anthocyanins might offer photoprotection during this period, a function comparable to that demonstrated for senescing leaves. Consistent with this, it has been demonstrated that anthocyanin accumulation reduces the severity of light stress (Li \& Cheng, 2008; Steyn, 2009; Steyn et al., 2009) and oxidative stress (Zhang et al., 2013a) in fruits, and prolongs the fruiting period (Zhang et al., 2013a), which is comparable to a prolonged senescence described in section 1.3.7. However, of the few studies that have investigated a physiological function in fruits, all have used fruit from domesticated varieties. Until the physiological significance of anthocyanin accumulation in fruits is tested in natural populations, its contribution to the evolution of fruit colour will remain unknown.

More poorly understood is the functional significance of anthocyanic reddening in the sterile components of reproductive organs like bracts, peduncles, rachides, rays and pedicels. Reddening in these organs is common for both species whose seeds are abiotically dispersed, and species whose seeds are biotically dispersed (Fig 1.4); however, the only studies to have investigated a function for anthocyanin accumulation in these structures have exclusively tested for a communicative role (Burns \& Dalen, 2002; Schaefer \& Braun, 2009). This role is thought to be one of frugivore attraction, where reddening either enhances the conspicuousness, or signals the quality of adjacent fruits. With regard to peduncles and pedicels, their primary function is to provide mechanical support, as well as water and carbohydrate transfer, to developing flowers and fruits, and as demonstrated for wheat, peduncles can make a significant contribution to photosynthesis themselves (Thorne, 1963; Thorne, 1965; Qin et al., 2009; Kong et al., 2010). Because these functions are comparable to those of vegetative organs, in particular stems, anthocyanin accumulation in peduncles and pedicels might similarly provide a function comparable to that seen in vegetative organs. 
Moreover, because peduncles and pedicels abscise after fruit removal, we can infer for those peduncles that turn red during fruit maturation that this colour change coincides with senescence. Anthocyanins might therefore perform a photoprotective function in peduncles, comparable to that seen in senescing leaves. 


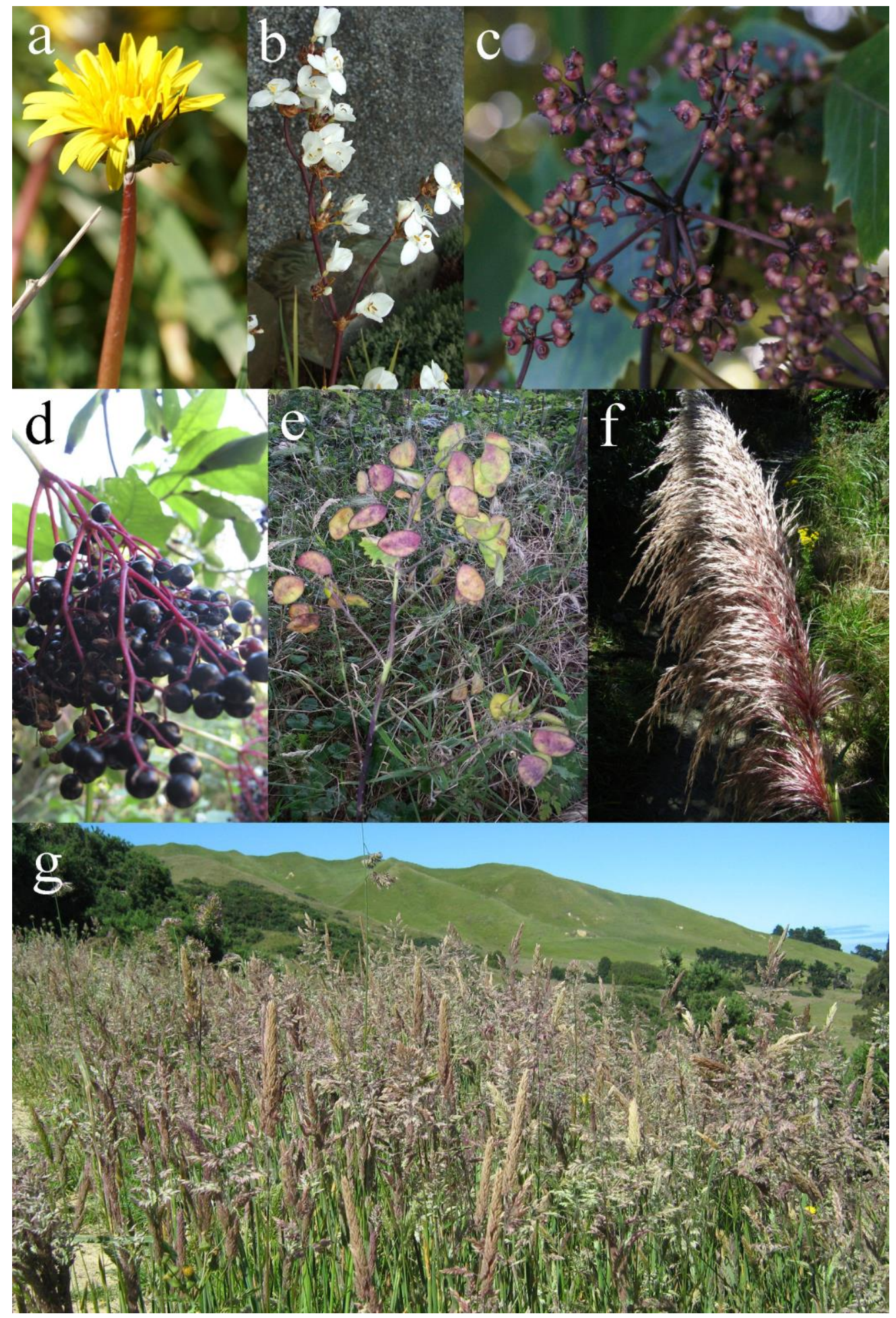

Fig 1.4. Photographs of red, purple or black peduncles (a-g), rays (b-g), rachides (b, e-g) and pedicels (b-g) among the reproductive structures of Taraxacum officinale (a), Libertia peregrinans (b), Pseudopanax arboreus (c), Sambucus nigra (d), Lunaria annua (e), Cortaderia jubata (f) and assorted grasses (g). Photograph credits: Hamish Carson (e) and Daniel Crossett (f). 


\subsection{Communicative functions in vegetative organs}

As mentioned in section 1.3.9, the communicative significance of anthocyanin accumulation has, until recently, been investigated exclusively in reproductive organs. Today, there are a number of hypotheses which similarly implicate a communicative role for anthocyanins in vegetative organs. There is indirect support for these various hypotheses, but direct support is lacking. Below I will review those hypotheses which have received the most attention, and discuss the empirical support that is required to demonstrate that anthocyanins provide a communicative function in vegetative organs.

\subsubsection{Fruit Flag hypothesis}

The fruit flag hypothesis proposes that foliar reddening might augment the conspicuousness of fruit displays, thereby enhancing seed dispersal (Stiles, 1982). Such enhanced conspicuousness can be achieved by either increasing the colour contrast of fruits against their surrounding foliage, or by increasing the size of a given display. However, this hypothesis has received mixed support. Facelli (1993) showed that more fruits were removed from Rhus gabra panicles if presented with artificial flags constructed from red plastic ovals, simulating senescing leaves. However, Li et al. (1999) found that fruit removal is maximal in winter for Rhus gabra, after autumn leaf senescence has been completed. It is therefore improbable that the benefit Facelli (1993) identified with plastic flags, can be applied to and subsequently explain, why senescing Rhus gabra leaves turn red.

In another study, Burns and Dalen (2002) compared the fruit colour and phenology of 10 North American species and found those species that fruit early in the year, when leaves are green, tend to produce red fruits, while those that fruit during autumn produce black fruits. The time dependent nature of this correlation argues against the fruit flag hypothesis and suggests that leaf colour is constrained by selective forces other than frugivores. For these species, it appears as though fruit colour had instead evolved to maximise its conspicuousness against senescing leaves. The significance of the fruit flag hypothesis on leaf colour evolution is therefore far from resolved, and for species where foliar reddening occurs independently of fruit maturation, the fruit flag hypothesis is inapplicable. 


\subsubsection{Anthocyanic pigmentation undermines herbivorous insect camouflage}

In 1988, Dicke and Sabelis identified an extraordinary method of herbivore defence in plants, whereby wounded plants reduce herbivory by emitting a volatile compound. This volatile compound would in turn, attract predators which feed on herbivorous insects (Dicke \& Sabelis, 1987). More recently, Lev-Yadun et al. (2004) suggested that non-green leaf colouration might provide a comparable function, but one in which plants use colour to expose the presence of herbivores. Green herbivores, normally camouflaged on green leaves, would be conspicuous when present on red leaves and their predators would more easily recognise them. Herbivores would in turn, avoid those plants on which their camouflage is undermined. This hypothesis would be equally applicable to whole leaf reddening, as well as localised patterns of anthocyanin production, as seen in the leaf midrib, veins or margins, of various species (see section 1.2.2). Localised reddening might be a means of reducing the cost of anthocyanin production, while still exposing herbivores which initiate feeding at those locations. Birds possess sophisticated colour vision (Bowmaker, 1980; Varela et al., 1993) and, as demonstrated on at least three tropical plant species, the presence of avian predators significantly reduces herbivore abundance and subsequent insect herbivory (Van Bael et al., 2003). However, no test to date has shown that non-green leaf colouration enhances herbivore detection and predation. Moreover, Schaefer and Rolshausen (2006) argued that leaf variegation can actually reduce insect detection. By providing a greater number of background colours, a plant provides camouflage to a more diverse array of insect herbivores. For these reasons, Lev-Yadun et al.'s (2004) hypothesis remains intriguing yet speculative.

\subsubsection{The coevolution hypothesis}

At the beginning of the 21st century, Hamilton \& Brown (2001) and Archetti (2000) proposed a radical new theory to explain the autumn reddening exhibited by the various deciduous plants. Termed 'coevolution', they suggest that this reddening serves as a warning to approaching aphid herbivores, whereby the healthiest plants advertise their vigour with an anthocyanic colour change. Those same plants, by virtue of their superior vigour, would be able to produce leaves that are less palatable, either by production of chemical defences that impair herbivore fitness, or by reducing the nutritional quality of their leaves. Aphids oviposit during autumn, and by avoiding those plants that appear the reddest, offspring that hatch the following season would be borne on the least defended trees (Archetti \& Brown, 2004). Coevolution therefore makes three distinct predictions; that non-green colouration signals 
reduced leaf palatability, that host selection by aphids is determined by leaf colour, and finally, those plants that deter aphid colonization incur a fitness advantage. To date, no study has satisfied all three criteria.

\subsubsection{Coevolution prediction 1: Are red leaves less palatable?}

The first requirement of the coevolution hypothesis is to demonstrate that red leaves are less palatable than green leaves (Archetti, 2000; Hamilton \& Brown, 2001; Archetti \& Brown, 2004). Unfortunately, there is little consensus as to what attribute of leaf quality autumn colour change might signal. Autumn colour change might, for example, signal a reduction in leaf nutrition, and thus senescing leaves would display an inverse relationship between redness and nitrogen content (Lee et al., 2003; Schaberg et al., 2003; Hughes et al., 2010b). Alternatively, redness might reflect an increased investment in anti-herbivory compounds. Although a specific compound which correlates to autumn leaf colour has yet to be identified, anthocyanin production in Cornus sanguinea and Symphoricarpus albus corresponded positively with total leaf phenolics (Karageorgou et al., 2008), a class of compounds, of which some display anti-feedant properties. Identifying the specific trait responsible for governing leaf palatability is an important requirement of the coevolution hypothesis. Without doing so, it cannot be tested whether this trait per se is responsible for improving plant fitness, as per prediction three (section 1.4.6).

Coevolution theory predicts that the characteristic which reduces leaf palatability comes at a cost to the plant; otherwise, all individuals would exploit it equally. Although some studies do not identify what trait determines leaf palatability, they do provide an indirect measure of

a plant's defensive investment by measuring proxies of plant health, such as fluctuating leaf symmetry (Hagen et al., 2004) or by identifying possible examples of resource allocation within the plant (Archetti, 2009b). For example, in mountain birch (Betula pubescens), the extent of autumn colouration, though not attributable to anthocyanins, was inversely proportional to fluctuating leaf asymmetry, an indicator of poor tree condition (Hagen et al., 2003; Hagen et al., 2004). The healthiest mountain birch, potentially the best defended, are therefore recognizable by leaf colour. However, it is unknown whether this colour reflects an increase in defensive compounds. In two other studies, the development and growth of moth larvae was impaired (Markwick et al., 2013), and the survival of aphids were reduced, when either were borne on Malus pumila (apple) individuals bearing red leaves (Archetti, 2009b). These data indicate that some aspect of red leaves is less palatable and is consistent with the 
theory that producing red leaves comes at a metabolic cost. However, these studies provide, at best, indirect support for coevolution.

\subsubsection{Coevolution prediction 2: Does leaf colour affect aphid behaviour?}

The second requirement of the coevolution hypothesis states that aphid colonization is determined by leaf colour. As expected, aphids are more abundant on green leaves compared to red (Furuta, 1986; Archetti \& Leather, 2005; Rolshausen \& Schaefer, 2007; Archetti, $2009 \mathrm{~b}$ ), though the role of colour in this preference requires further resolution. Aphids may prefer a characteristic specific to green leafed plants which is independent of colour itself, such as the composition of volatile organic compounds (Rolshausen \& Schaefer, 2007), or environmental attributes, such as light or temperature, exclusive to green-leaved plants. To isolate the extent to which colour influences aphid behaviour, Schaefer and Rolshausen (2007) manipulated Sorbus aucuparia leaf colour to appear either red or green, and then they quantified aphid colonization. Curiously, aphids showed no preference for leaf colour, but colonization correlated positively with a plant's previous fruit set. In this example, colonizing aphids demonstrate a non-random process of host selection, but the process is influenced by traits other than colour. In another study, Doring et al. (2009) compared aphid visitation between traps that differ only by colour. Seventy different colours were used in this study and by applying a model of aphid vision to each, the authors calculated how each colour was perceived by aphids. Interestingly, aphid visitation was proportionate to the perceived ratio of green: blue light that a trap reflected. Hues we perceive as green, yellow or blue were favoured, whereas hues we perceive as red were avoided. Anthocyanins, by absorbing green light, are especially effective at altering the ratio of green: blue reflected light, and thus, altering a leaf's perceived attractiveness to aphids. Doring et al. (2009)'s study represented a significant advancement in coevolution research, showing that colour influences aphid behaviour. If it can be shown that this response to trap colour is similarly present in leaf selection by aphids, the second requirement of the coevolution hypothesis will be satisfied.

\subsubsection{Coevolution prediction 3: Does signalling leaf palatability provide a selective advantage?}

Avoiding herbivory might not necessarily mean that a plant incurs a selective advantage. For example, producing nitrogen-poor leaves might be a strategy employed by plants to reduce leaf palatability, or it might be a symptom of ill-health. Under both scenarios these leaves 
avoid herbivory, but only under the first scenario would we expect to see an increase in fitness associated with nitrogen deficiency. For this reason, the third requirement of the coevolution hypothesis states that by deterring herbivory, plants receive a selective advantage. In practical terms, this prediction can be the hardest to test, especially if predictions one and two are yet to be fulfilled.

Fitness reflects an organism's lifetime reproductive output, and by definition, those species that undergo autumn senescence have life spans that exceed one year. Theoretically, an investment in metabolic resources towards herbivore defence may result in a short-term reduction in reproductive output, but a long-term gain in an individual's fitness. In mountain birch, Hagen et al. (2003) found that early autumn colouration, though not attributable to anthocyanins, negatively correlated to aphid colonization, as well as catkin production. In this case, those plants that were the most successful at deterring herbivory, displayed the lowest reproductive output. Although this observation is counter to that expected under coevolution, it may be that an investment which reduces herbivory, comes at an immediate reproductive cost, but improves reproductive output when measured over the course of a plant's lifetime.

Because testing the above scenario remains difficult, Archetti (2009b) proposed an inventive alternative utilizing domesticated apple (Malus pumila) varieties. He stated, "fruit trees under domestication have been artificially selected for productivity and taste, irrespective of their autumn colours, and they have not been under natural selection against insects. While cultivated trees are still attacked by parasites, their fitness and reproduction does not ultimately depend on their reaction to insects". Archetti (2009b) claimed that by comparing autumn colouration between wild and cultivated varieties of apple, he identified a long-term experiment on leaf colour under differing levels of herbivore pressure. As expected, the proportion of individuals with red senescing leaves was indeed higher for a wild population of apple plants, compared to a cultivated population. While the approach used in this study was novel, the assumption on which it was based may not be entirely true, namely, that for crop plants "fitness and reproduction [do] not ultimately depend on their reaction to insects" Archetti (2009b). Coevolution predicts that avoiding herbivory imparts a fitness advantage, presumably because additional resources become available for reproduction. Archetti's (2009b) study neglected to acknowledge that an individual's fitness, (i.e., crop yield) is also a significant trait on which artificial selection acts; any individual with insufficient herbivore defences would incur an increase in herbivory, a decrease in yield, and would unlikely be propagated. In this sense, artificial selection would favour traits that deter herbivory in much 
the same way that natural selection does (a notable exception to this would be if cultivated varieties are treated with insecticide over successive generations). Interestingly, this study also found that among varieties of cultivated apple, those that bore red leaves during senescence were the varieties most susceptible to fire blight, an insect-borne disease. Archetti (2009b) interpreted this result as a possible outcome of coevolution, i.e. foliar reddening is maintained in those populations most in need of avoiding herbivores, thereby acknowledging that herbivore pressure has the potential to influence the evolution of leaf colour in cultivated populations. It appears as though the results of this study were interpreted inconsistently; some were viewed under the assumption that herbivore pressure influences leaf colour evolution in apple cultivars, while other results were interpreted assuming it does not. Regardless, without quantifying the extent to which foliar signalling improves plant fitness, support for the third prediction of the coevolution hypothesis remains indirect and weak.

\subsubsection{Those signalling roles purportedly subsumed by coevolution}

The introduction of the coevolution hypothesis by Hamilton and Brown (2001), and Archetti (2000) was followed by a number of alternative hypotheses which similarly implicated a communicative role for red leaves. Many of these other hypotheses have now been subsumed by Hamilton and Brown's (2001) and Archetti's (2000) coevolution hypothesis (Archetti, 2009a). Although my thesis may not test the specific requirements that each hypothesis makes, it is useful to recognise the predictions of each hypothesis, and discuss whether their reclassification as coevolution is justified.

\subsubsection{Defence indication}

The flavonoid biosynthetic pathway, from which anthocyanins are derived, leads to the production of a diverse group of compounds of which some are involved in plant defence. Tannins for example, display well-known anti-herbivory properties (Bernays, 1981; Bernays et al., 1989; Barbehenn \& Peter Constabel, 2011), and share a common precursor with anthocyanins, leucoanthocyanidin (Peters \& Constabel, 2002). Schaefer and Rolshausen (2006) suggested that by sharing this biosynthetic pathway, anthocyanin production, which may have evolved in response to physiological pressures, is inherently linked to that of defensive compounds. They argued that leaf reddening may be an inherent by-product of defensive investment, and termed the relationship 'defence indication'. Schaefer and Rolshausen's hypothesis suggests the relationship between leaf colour and defences need not 
be maintained by herbivore pressure. In contrast, 'coevolution' suggests that herbivore pressure is directly responsible for the evolution of red leaves. Unfortunately, it has been argued by some reviewers that 'defence indication' be subsumed by 'coevolution' (Archetti, 2009a; Archetti et al., 2009). The strength and clarity of each hypothesis is only realised when they are kept distinct. For example, defence indication may explain how a relationship between leaf colour and defensive compounds first evolved, and coevolution may explain the subsequent evolution of leaf colour, once herbivore pressure became a selective force.

\subsubsection{Signal that leaves are about to be shed}

To deter approaching herbivores, Lev-Yadun and Gould (2007) suggested that autumn colouration may signal that a leaf is about to be shed. Herbivores would, in turn, avoid those non-green leaves, and the plant would benefit by deterring gravid female herbivores. However, Archetti and Brown (2004) had already described this relationship as fulfilling the requirements of the coevolution hypothesis. They argued that early initiation of senescence may be the trait by which a plant reduces leaf palatability, and as required by coevolution theory, comes at a metabolic cost to the plant through a reduction in carbon assimilation. According to Archetti and Brown (2004), this hypothesis need not be restricted to red leaves, and may similarly apply to those that turn yellow, orange or brown when they senesce.

\subsubsection{Aposematic signalling}

Aposematic signals are commonplace in the animal kingdom, the most recognisable examples being the yellow and black stripes of many wasps and the spotted patterns of many ladybirds (Poulton, 1890; Merilaita \& Ruxton, 2007). In plants, comparable examples are rare, but aposematism may explain the conspicuous reddening seen in the mechanical defences that plants employ (Lev-Yadun, 2001; Lev-Yadun, 2003a). Foliar reddening may also be an aposematic signal, and a means of warning approaching herbivores of a plant's chemical defences. In this sense, an aposematic signal is comparable to that described under coevolution, and the terms are often used synonymously (Archetti, 2009a). However, aposematic signals are those which enhance recognition of a well-defended species, and are by definition, conspicuous. Signal honesty under aposematism is maintained because this added conspicuousness is detrimental for dishonest signallers, especially when abundant within a population. In contrast, those signals which evolve under coevolution need not be 
conspicuous, and signal honesty is maintained under coevolution because signalling itself is costly (Archetti, 2000; Hamilton \& Brown, 2001).

\subsubsection{Extension of the coevolution hypothesis to non-senescing organs}

Although originally proposed to explain foliar reddening during autumn senescence and the subsequent behaviour of aphids during oviposition, coevolution might similarly explain the reddening displayed by non-senescing leaves. In some plants which accumulate anthocyanins during leaf development, the extent of reddening correlates negatively with leaf herbivory and positively with total leaf phenolics, a class of compounds, of which some display antifeedant properties (Karageorgou \& Manetas, 2006; Karageorgou et al., 2008). In contrast, these correlations were absent for four other species whose young leaves turn red (Karageorgou et al., 2008). Other studies have investigated a signalling role for patterns of anthocyanin accumulation in which reddening is restricted to only a portion of the lamina. In Columnea consanguinea, reddening occurs as a spot on the abaxial surface of the leaf and the larger the spot, the less herbivory a leaf incurs (Wong \& Srivastava, 2010). For some species within the genus Veronica, foliar anthocyanins are restricted to the leaf margin, presenting a red border around an otherwise green lamina. Hughes et al. (2010b) compared the leaf phenolic content and extent of herbivory between Veronica spp. that possess red leaf margins and those that do not, and found no support for coevolution.

Support for coevolution is evidently mixed, both when the theory is applied to senescing leaves and when applied to non-senescing patterns of foliar reddening. At best, it has been shown that anthocyanin accumulation reflects phenolic content in leaves (Karageorgou \& Manetas, 2006; Karageorgou et al., 2008), and colour has the potential to alter herbivore behaviour (Doring et al., 2009). However, there are many purported functions for phenolic compounds quite aside from the role of some of them in plant defence (Lawler et al., 1998; Lawler et al., 1999; Close \& McArthur, 2002), and it is yet to be shown that colour is responsible for herbivore leaf selection. To satisfy the first two predictions of coevolution, it must still be shown that leaf colour reflects an investment in a specific trait that reduces leaf palatability, and that herbivores use colour in leaf selection. Coevolution remains the most well-known example of a communicative role for anthocyanic colouration in vegetative organs, and may potentially explain the patterns in non-green leaf colouration that many plants display. However, without first satisfying predictions one and two, the final prediction of coevolution, that signalling leaf defences improves plant fitness, cannot be directly tested. 


\subsection{Addressing the dichotomy in anthocyanin research}

It has been suggested that anthocyanins impart a variety of physiological benefits in vegetative organs; these same benefits may similarly apply to reproductive organs. Conversely, the communicative potential of anthocyanic colouration has been realised in reproductive organs for over a century, yet only recently postulated for vegetative organs. As yet, no study has tested for a physiological function for anthocyanins in the reproductive organs of a naturalised species, and no study has unequivocally demonstrated a communicative function for anthocyanins in vegetative organs. Anthocyanins have the potential to perform multiple functions in plants, perhaps simultaneously. If we do not address this dichotomy in anthocyanin research, we cannot know what selective pressures are responsible for driving the evolution of anthocyanic colouration in plant organs, whether they be vegetative or reproductive.

In the following chapters of this thesis I will rectify this imbalance in anthocyanin research, first by testing for a signalling role in vegetative organs, and then by investigating a physiological role in reproductive organs.

\subsubsection{Pseudowintera colorata is a model species to test for a communicative function in vegetative organs.}

The New Zealand pepper tree, Pseudowintera colorata, is for several reasons, a particularly useful model to test for a communicative function for anthocyanins in vegetative organs, that being, whether anthocyanic reddening signal's chemical defences in leaves. First, $P$. colorata leaves bear conspicuous red (anthocyanic) margins and the extent of margin size varies considerably among individuals. Second, the primary defence compound in P. colorata is known; it is the sesquiterpene dialdehyde polygodial, which imparts a pungent, peppery taste and has potent insect anti-feedant properties (Barnes \& Loder, 1962; Asakawa et al., 1988). Furthermore, polygodial content is also variable among individuals, presenting us with an opportunity to compare the extent of leaf reddening to concentrations of a specific defence compound. Finally, a list of $P$. colorata herbivores has been documented (http://plantsynz.landcareresearch.co.nz/; accessed August 2014), which means we can not only compare herbivory rates for red- and green-margined leaves in a natural P. colorata population, we can also conduct feeding trials under controlled laboratory conditions using natural herbivores. 
In chapter 2, I tested the requirements of a signalling role for red (anthocyanic) leaf margins in $P$. colorata. I hypothesised that polygodial concentrations in leaves with red margins would be greater than those with green margins, and leaves with the larger red margins would incur less herbivory. I also tested whether the brown-headed leaf roller moth (Ctenopseustis obliquana), a natural edge-feeding generalist herbivore of $P$. colorata, responds to the red leaf margins as a visual signal, and avoid the more strongly defended plants. I hypothesised that $C$. obliquana adults would prefer to oviposit and larvae would prefer to eat, greenmargined leaves over red-, but only under light regimes which permit discrimination of leaf colour.

\subsubsection{Sambucus spp. are the model species to test for a physiological function in reproductive organs.}

Elderberry is the common name given to various species within the genus Sambucus. For at least two of these species, S. nigra and S. canadensis, fruit maturation is preceded by anthocyanin accumulation in the subtending peduncles, rays and pedicels. This accumulation induces a colour change from green to red, the extent of which varies considerably between individuals as well as between infructescences on the same plant, and is present throughout the fruiting period. For $S$. nigra, a communicative function (i.e. peduncle reddening signals adjacent fruit quality and promotes frugivore visitation) for this colour change has been investigated. Schaefer and Braun (2009) showed that the extent of peduncle reddening positively correlates to fruit sugar content in S. nigra, and on infructescences bearing red peduncles, frugivory was higher than on infructescences bearing green peduncles. However, Schaefer and Braun (2009) also identified some characteristics that suggest a communicative role may not be the primary function of peduncle anthocyanins. Anthocyanin accumulation precedes fruit maturation and is only present on those infructescences exposed to high light. If this was a signalling adaptation, exposed peduncles would be unnecessarily red while fruits are still unripe, and shaded peduncles would remain unnecessarily green.

It may be that anthocyanin accumulation in $S$. nigra peduncles is under selection pressures beyond those exerted by frugivores. Because reddening precedes abscission in both S. nigra and $S$. canadensis peduncles, we know that anthocyanin accumulation coincides with senescence, a period when peduncles may be especially susceptible to light stress. In senescing leaves, anthocyanin accumulation has been implicated in a number of physiological benefits including a reduction in light stress (see section 1.3.1), an improved nitrogen 
resorption (see section 1.3.5) and a prolonged senescence (see section 1.3.7). It may be that anthocyanins perform physiological functions in peduncles, comparable to those described for leaves yet this has not been tested. Reddening in Sambucus spp. therefore provides us with an ideal opportunity to test whether anthocyanins perform a physiological function in reproductive organs, and whether these functions occur simultaneously with those communicative functions already proposed.

In chapter 3, I tested the requirements of both a communicative role and a light screening role for anthocyanins in S. nigra peduncles. To test the requirements of a light screening role, I monitored an entire season's fruit removal for infructescences of varying peduncle redness, and compared peduncle colour for infructescences whose crop size had been experimentally manipulated. According to my communication hypothesis, peduncle reddening would enhance fruit removal and would be proportionate to reward quality (i.e. crop size). To test for a photoprotective role for anthocyanins in S. nigra peduncles, I hypothesized that exposure to full sunlight would be the requirement for anthocyanin accumulation and the presence of anthocyanins would both reduce transmittance of visible light to chloroplasts and improve the quantum efficiency of photosynthesis under high light. Finally, I hypothesised that anthocyanins would be responsible for reducing photoinhibition following light stress.

In chapter 4, I tested the requirements of the hypothesis 'that anthocyanins prolong senescence in S. canadensis peduncles'. Senescence is characterised by a number of processes including the catabolism of chlorophyll and xanthophyll, a reduced ability to mitigate light stress and progression of an abscission layer where peduncles attach to the plant. Firstly, I compared, for red and green peduncles, the rate at which chlorophyll and xanthophyll concentrations, and photosynthetic health, as estimated by chlorophyll fluorescence, decline during senescence. Secondly, I compared the tensile strength of red and green peduncles, an indicator of abscission layer progression in senescing tissues (dela Fuente \& Leopold, 1968; Schaberg et al., 2008). Lastly, I used a novel experimental design to compare longevity, lipid peroxidation and nitrogen resorption between green peduncles bearing a transparent light filter, and green peduncles bearing a fake anthocyanic light filter. This uniquely produced two treatments with identical developmental histories that differed only by the light profiles transmitted to peduncle tissues. Collectively, these studies will redress a distinct gap in our current understanding of anthocyanin function. 


\section{Chapter 2: Red leaf margins indicate increased polygodial content and function as visual signals to reduce herbivory in Pseudowintera colorata.}

\subsection{Abstract}

Leaf reddening may serve as a warning signal to approaching herbivores, but experimental support is lacking. Here, I hypothesised that red leaf margins reduce leaf herbivory by signalling to herbivorous insects the presence of increased chemical defences. Leaves were collected from a natural population of Pseudowintera colorata. Red margin size, herbivory damage, anthocyanin content, and concentrations of polygodial, a sesquiterpene dialdehyde with anti-feedant properties, were quantified. Feeding trials involving larvae of Ctenopseustis obliquana, a generalist herbivore, were conducted on red- and green-margined $P$. colorata leaves in darkness, or under white, green or red light. Polygodial concentrations were 33\% higher for red-margined leaves compared to green-margined leaves and red margin size negatively correlated with natural herbivory $\left(\mathrm{r}^{2}=0.21\right)$. In trials under white light, $C$. obliquana consumed 130\% more green- than red- margined leaf laminae. Larvae exhibited no feeding preference when light was manipulated such that leaf colour discrimination was impaired. This study shows that red leaf margins provide a reliable and effective visual signal of chemical defence in P. colorata. Moreover, C. obliquana larvae perceive and respond to the colour of the leaf margins, rather than to olfactory signals. This study provides direct experimental evidence for aposematic colouration in red leaves.

\subsection{Introduction}

Anthocyanin pigments in plant reproductive organs have long been known to serve as a visual signal to attract animals, promoting pollination and/or seed dispersal (Gould et al., 2009). In vegetative organs, however, anthocyanins might be deployed to repel animals. For example, to explain the autumnal reddening in the leaves of many deciduous trees, Archetti (2000) and Hamilton and Brown (2001) suggested that anthocyanins function as a visual warning to deter browsing by insect herbivores. Accordingly, red leaves would signal enhanced investment in defensive compounds that impair insect fitness. The signal would in turn be used by herbivores to select which plant to colonise. Aphids that switch host in autumn would colonise the least defended plants, and aphid infestation would be reduced in the redder, more strongly defended plants (Archetti, 2009a). This so-named 'coevolution hypothesis' has received indirect support in recent years from a variety of studies (Hamilton 
\& Brown, 2001; Hagen et al., 2003; 2004; Archetti \& Leather, 2005; Karageorgou et al., 2008).

Although originally proposed to explain the reddening or yellowing of the entire lamina in senescing leaves, Archetti's (2000) and Hamilton and Brown's (2001) hypothesis might similarly apply to more localised patterns of anthocyanin accumulation in non-senescing leaves (Lev-Yadun et al., 2002). In many plants, anthocyanic pigmentation is restricted to the stipules, petiole, major veins, trichomes, marginal teeth, and/or leaf apex (Wheldale, 1916; Hatier \& Gould, 2009). Of particular interest in relation to animal communication are the anthocyanins in the epidermal and/or sub-epidermal cells at leaf margins. These can provide a prominent red border that presents a sharp chromatic contrast against the green lamina (Fig 2.1a-d). Although there has been no published systematic survey of species bearing leaves with red margins, they are evidently very common. Red margins are often used by taxonomists as a character for species identification (Bayly \& Kellow, 2006; Versieux \& Wanderley, 2007; Redden, 2008), and as a marker to study genetic linkages in crop species as diverse as maize (Flint-Garcia et al., 2005), banana (Jarret et al., 1993), lettuce (Sabharwal \& Doležel, 1993), mustard (Nick et al., 1993) and rice (Hadagal et al., 1981). There are also numerous reports of anthocyanic leaf margins being induced in response to mineral nutrient deficiencies (Walker, 1956; Johanson \& Walker, 1963; Nyborg \& Hoyt, 1970; Haque \& Walmsley, 1973; Balo et al., 1975; Hassouna, 1977; Raese, 2002).

The function (if any) of anthocyanins at the leaf margin is unknown, but their extremely restricted histological distribution within the leaf argues against many of the physiological hypotheses for foliar anthocyanins, such as photoprotection and antioxidant activities (Gould, 2004; Hatier \& Gould, 2009). However, because many herbivores initiate feeding at the leaf edges, it is at least possible that the marginal anthocyanins function as a visual signal to indicate that the leaves contain unpalatable compounds. Edge-feeding is prominent among orthopteran, coleopteran and lepidopteran larvae (Bernays, 1998), and the ability to detect red hues is well established among members of Lepidoptera and Coleoptera (Briscoe \& Chittka, 2001).

In the process of edge-feeding, a plant loses not only those resources contained within the leaf portion eaten, but also the future photosynthetic potential of that portion. A damaged margin enhances evaporative water loss and could place water stress on the adjacent tissues as well as increase risk of infection by pathogens. Thus, edge-feeding is costly, and can be 
more detrimental than other types of herbivory, such as phloem feeding, wherein the leaves are left relatively intact. Relative growth rates of Solidago altissima, for example, were shown to be significantly reduced by edge-feeding beetles, but were unaffected by sapfeeding aphids (Meyer, 1993). It is not surprising, therefore, that secondary metabolites which are considered to be involved in defence against insect herbivores are often more concentrated at the periphery than in interior regions of a leaf lamina (Gutterman \& ChauserVolfson, 2000; Kester et al., 2002; Shroff et al., 2008; Hughes et al., 2010b). Anthocyanins may provide the means for a plant to advertise defensive compounds in areas where herbivores prefer to initiate feeding.

To my knowledge, only one previous study has thus far addressed a possible anti-herbivory role for red leaf margins. Hughes et al. (2010b) compared insect damage and leaf phenolic content across 11 Veronica species which differed in leaf margin colour. Contrary to the authors' hypothesis, the presence of anthocyanins did not correspond with increased phenolic content at the leaf margins. However, the authors acknowledged limitations to their dataset since (i) all plants had been growing together in a common garden, and were not, therefore, subjected to natural herbivory pressure, and (ii) the study did not account for interspecific differences in leaf structure and biochemistry. Moreover, measurements of total phenolic content may not be the best estimate of defensive strength; the primary role of many phenolics appears to be to protect leaves from photodamage rather than from herbivory (Close \& McArthur, 2002), and the phenolics which are known to be involved in defence constitute only a small fraction of the total phenolic pool (Lawler et al., 1998; Lawler et al., 1999). Hughes et al. (2010b) therefore recommended that future studies focus on specific defensive compounds, rather than general phenolic pools, to explore a possible defensive role of anthocyanic leaf margins, and to utilise intra-specific systems for better control of variation in leaf structure and chemistry. Thus, the hypothesis that red leaf margins reduce insect herbivory remains a possibility which warrants further investigation.

The New Zealand pepper tree, Pseudowintera colorata (Raoul) Dandy (Winteraceae), is for several reasons a particularly useful model to test for defensive functions of red leaf margins (Perry \& Gould, 2010). First, populations exhibit pronounced variation in leaf margin colour and size among individuals (Fig 2.1; P. colorata leaves also develop red margins around areas of mechanical damage (Gould et al., 2002a) but this induced colouration was not a factor in the present study). Second, the primary defence compound in P. colorata leaves is known; it is the sesquiterpene dialdehyde polygodial (Fig 2.1e), which imparts a pungent 
taste and has potent insect anti-feedant properties (Barnes \& Loder, 1962; Asakawa et al., 1988). In vitro studies have shown polygodial to be an effective anti-feedant in concentrations of $3 \mathrm{mg} \mathrm{g}^{-1} \mathrm{WW}$ (Gerard et al., 1993), whereas $P$. colorata leaves contain at least $10 \mathrm{mg} \mathrm{g}^{-1}$ (Wayman et al., 2010). Finally, P. colorata herbivores have been documented (http://plant-synz.landcareresearch.co.nz/; accessed August 2014). Thus, P. colorata presents an opportunity both to collect correlative data on margin colour, herbivory damage, and polygodial content, and to conduct feeding trials under controlled laboratory conditions using natural herbivores.
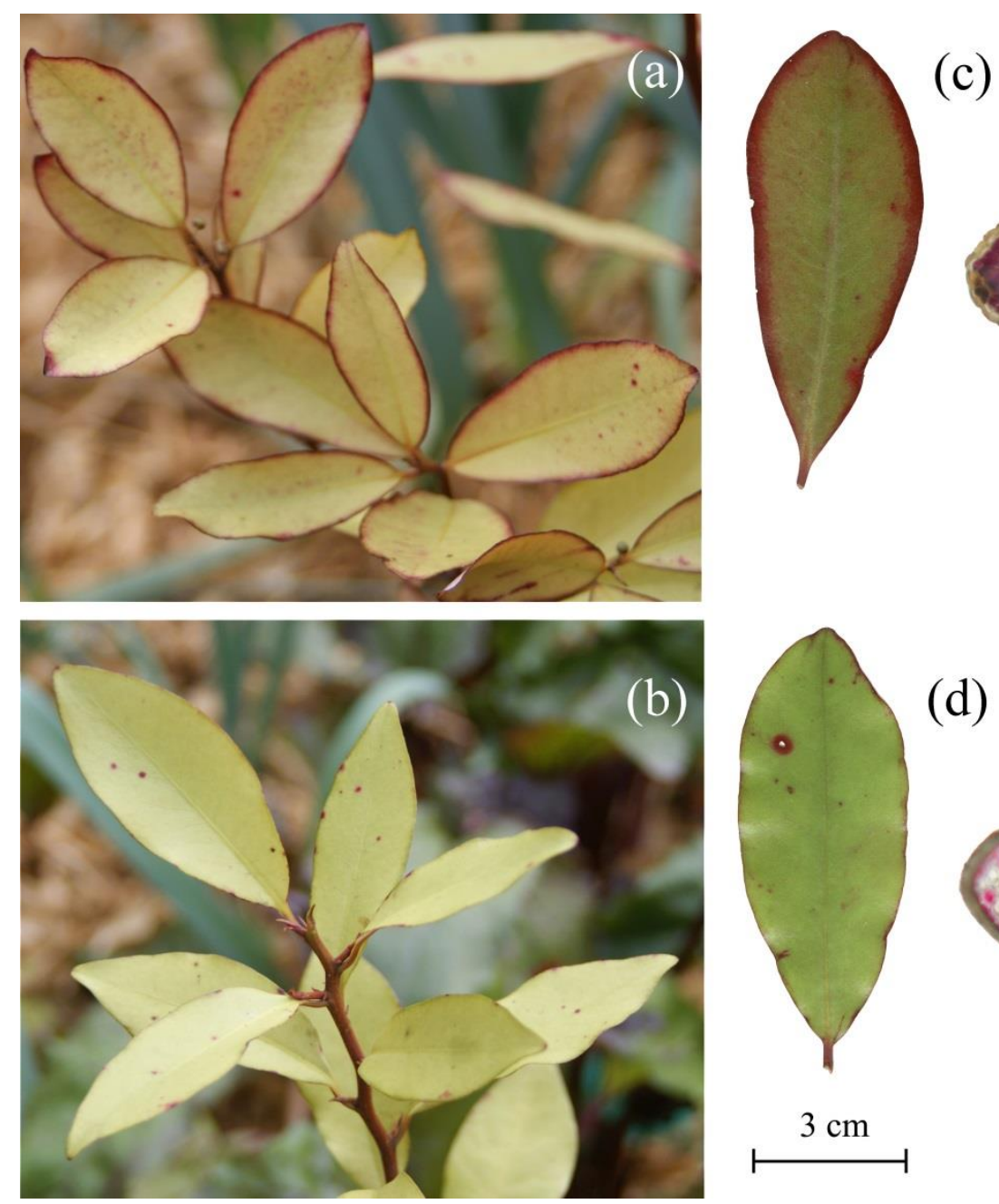
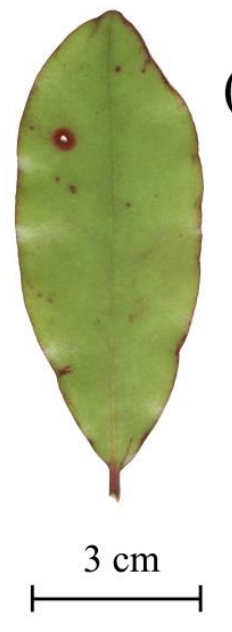

(e)
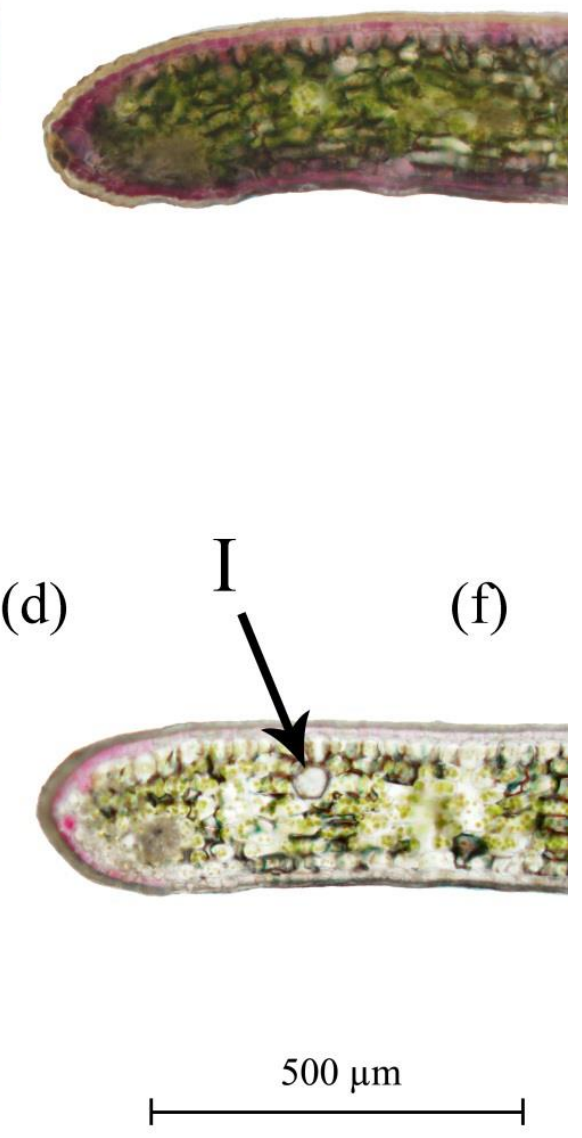

Fig 2.1. Red- (a, c, f) and green- (b, d, g) margined leaves of Pseudowintera colorata: (a, b) photographs of individual branches; (c, d) photographs of leaf laminae; (e) polygodial chemical structure; (f, g) photomicrographs of transverse sections. I, idioblast.

In this study I tested the fundamental requirement of any visual signal - that red leaf margins in $P$. colorata provide a reliable indication of the defensive status of a plant. I hypothesised that (i) polygodial concentrations would be highest around the leaf margin; (ii) polygodial 
concentrations in leaves with red margins would be greater than those with green margins, and (iii) leaves with the larger red margins would incur less herbivory. I also tested whether a natural edge-feeding generalist herbivore of $P$. colorata, the brown-headed leaf roller moth (Ctenopseustis obliquana) responds to the red leaf margins as a visual signal, and avoids the more strongly defended plants. I hypothesised that $C$. obliquana larvae would prefer to eat, and adults to oviposit, on leaves with green margins, but only under light conditions in which colour discrimination was possible. Leaf images used in section 2.3.2 and leaf chemistry data in section 2.3.3 were generated as part of my 2010 Honours dissertation and analysed independently here.

\subsection{Materials and Methods}

\subsubsection{Plant Material}

P. colorata leaves were collected between 2010 and 2011, in the Otaki Forks region of the Tararua Forest Park $\left(40^{\circ} 54^{\prime} 27.8^{\prime \prime} \mathrm{S}, 175^{\circ} 15^{\prime} 21.1^{\prime \prime} \mathrm{E}\right)$ in New Zealand, an area of montane forest (altitude $860 \mathrm{~m}$ ) dominated by Nothofagus spp. In this population, P. colorata is a prominent understory tree and a polygodial-rich chemotype (Wayman et al., 2010). Random sampling was achieved using six parallel transect lines, $10 \mathrm{~m}$ apart and running northeast to southwest. Each transect was $50 \mathrm{~m}$ long and spanned a range in elevation not greater than 20 m. P. colorata plants within $2 \mathrm{~m}$ of each transect were sampled if the plants were (i) at least $10 \mathrm{~m}$ from the nearest walking track; (ii) between 1.25 and $1.75 \mathrm{~m}$ tall; and (iii) at least $3 \mathrm{~m}$ away from any previously sampled plants. A lateral branch bearing at least seven fully expanded leaves was chosen at random and excised from each of 98 plants. These branches were refrigerated within four hours of collection.

\subsubsection{Margin width and herbivory measurement}

Digital images of the abaxial and adaxial surfaces of leaves from nodes 1, 3 and 7 were captured using a Canon CanoScan 8400F flatbed scanner (Tokyo, Japan). Nodes were numbered basipetally from the youngest fully expanded leaf. Images were processed using GIMP v2.6.7 (http://www.gimp.org/) to highlight all red pixels with values between 240 and $255 \mathrm{R}$ units in the RGB colour model. The images were exported into ImageJ v1.41 (National Institute of Health, Bethesda, MD, USA) and used to measure the width of a red margin at a point normal to the centre of the midrib. If that point had been removed by herbivory, the nearest portion of remaining margin was measured. Because the width of red margin was 
relatively uniform around each leaf, a single measurement of margin width was adequate for each leaf.

To quantify herbivory damage, the residual surface area of each leaf lamina was measured using ImageJ. Adobe Photoshop 5.0 (San Jose, CA, USA) was used to recreate the original leaf shape by filling in areas lost to herbivory. If the herbivory damage was too extensive for a confident reconstruction, the original leaf shape was estimated from comparison with that of the adjacent leaf along the branch from which it had been removed. Once the preherbivory leaf shape had been reconstructed, its lamina area was again measured using ImageJ. The amount of herbivory was calculated as the difference between pre- and postherbivory surface areas.

\subsubsection{Anthocyanin and polygodial quantification}

A further $20 P$. colorata individuals were sampled on the same date as above, 10 of which bore leaves with red margins and 10 with green margins. Because of constraints on the numbers of leaves that could be sampled, I restricted the chemical analysis to those leaves for which margin size was at the upper and lower extremes of the range. A leaf margin was designated as red if its anthocyanic pigmentation at the leaf's widest point extended beyond $10 \%$ of the lamina width, and designated as green if anthocyanins extended less than $2 \%$ of the lamina width. The samples excluded leaves that had previously incurred herbivory. Fully expanded leaves from node 1 were removed, the margins excised from the interiors, and both margin and interior were frozen at $-80{ }^{\circ} \mathrm{C}$. Margins were dissected from the most peripheral 5 $\mathrm{mm}$ of the leaf's entire circumference. Samples were freeze dried for $24 \mathrm{~h}$, ground to a powder using a mortar and pestle, and sent to the New Zealand Institute for Plant \& Food Research Ltd, Dunedin, for quantification of polygodial and anthocyanin concentrations by HPLC using a procedure modified after Wayman et al. (2010). Leaf subsamples ( 10 mg) were extracted with rectified spirits $(1 \mathrm{ml})$ containing $\mathrm{C}_{10}$ anilide $(200 \mu \mathrm{g})$ as an internal standard (Perry et al., 1996a), briefly sonicated ( 30 s), stirred overnight, and then filtered through a $0.45 \mu \mathrm{m}$ PTFE filter. Analyses were performed on an Agilent 1100 HPLC (Agilent Technologies) fitted with a diode array detector, using a Luna (II) $250 \times 3 \mathrm{~mm}$ RP-18 (5 $\mu \mathrm{m})$ column (Phenomenex, Torrance, CA) with a SecurityGuard ${ }^{\mathrm{TM}} 4$ x 2 mm C18 guard column (Phenomenex, Torrance, CA) at $30^{\circ} \mathrm{C}$. Peaks were monitored at 206, 230 280, 330 and 530 $\mathrm{nm}$. The mobile phase was $\mathrm{MeCN}$ in $\mathrm{H}_{2} \mathrm{O}$, both containing formic acid $(0.1 \%): 5 \% \mathrm{MeCN}$ at $0 \mathrm{~min}, 100 \%$ at $30 \mathrm{~min}, 5 \%$ at $35 \mathrm{~min}, 5 \%$ at $40 \mathrm{~min}$. The flow rate was $1.0 \mathrm{ml} \mathrm{min}^{-1}$ with 
injection volumes of $5 \mu \mathrm{l}$. Quantification of polygodial concentrations was based on calibration with an isolated reference sample of polygodial against the internal standard, with detection at $230 \mathrm{~nm}$. Quantification of anthocyanins was achieved by calibrating with a commercial source of cyanidin-3-O-glucoside (Extrasynthese, France) at $530 \mathrm{~nm}$ versus the internal standard at $206 \mathrm{~nm}$. Total anthocyanins were reported as cyanidin-3-O-glucoside equivalents.

\subsubsection{Leaf microscopy and reflectance}

Transverse hand sections through the interior and margin of fresh leaf material were examined in an Olympus AX70 photomicroscope (Olympus Optical Co., Hamburg, Germany) and photographed using an Olympus DP70 digital camera (Olympus Optical Co., Hamburg, Germany).

Leaf reflectance was measured using an Ocean Optics (Dunedin, FL) USB2000 spectrometer fitted with a PX-2 pulsed xenon light source. Five red- and five green-margined leaves, all from node 1 of different plants, were detached and their reflectances measured at the leaf margins. The criteria used to categorise leaf margin colour were the same as those used in the polygodial quantification. Light was provided at $45^{\circ}$ to the leaf's adaxial surface and diffuse reflectance measured at $0.4 \mathrm{~nm}$ intervals from 400 to $700 \mathrm{~nm}$, and referenced to an Ocean Optics WS-1 diffuse reflectance standard.

\subsubsection{Feeding and oviposition trials}

Ctenopseustis obliquana larvae and pupae were purchased from The New Zealand Institute for Plant \& Food Research, Auckland. All had been raised in captivity on a general purpose diet (Singh, 1983). A third-instar larva, 8-10 $\mathrm{mm}$ in length, was placed into each of 136 Petri dishes (15 cm diameter) containing one green- and one red-margined P. colorata leaf. The criteria used to categorise leaf margin colour were the same as those used in the polygodial quantification. Leaves were harvested using the sampling strategy described above and stored overnight at $4{ }^{\circ} \mathrm{C}$ in a plastic bag on a moist paper towel. Both leaves in each dish were similar in size and shape, free of edge-feeding herbivory damage, and originated from node 1. Leaves were scanned to measure their surface areas using ImageJ, and placed $3 \mathrm{~cm}$ apart within the Petri dish (Fig 2.2). One larva per dish was placed equidistant from the two leaves. Feeding trials were conducted at $18^{\circ} \mathrm{C}$ in darkness, or under $25 \mu \mathrm{mol} \mathrm{m}{ }^{-2} \mathrm{~s}^{-1}$ white, green, or red light, using 34 replicates per treatment. Light was provided by Phillips $36 \mathrm{~W}$ cool white fluorescent tubes, the colour and intensity manipulated using Rosco Supergel (Stamford, 
USA) plastic filters \#398 (neutral density), \#389 (green; maximum transmittance 500-520 $\mathrm{nm}$ ), and \#19 (red; maximum transmittance $>620 \mathrm{~nm}$ ). The dark treatment was obtained by shielding the dishes with opaque polyvinyl. After $72 \mathrm{~h}$ the leaves were rescanned and postherbivory surface area was calculated using ImageJ, from which, leaf area eaten was calculated. Additionally, the distribution of herbivory was identified by dividing each leaf image into five portions, the first representing the central leaf lamina, and the subsequent four portions each representing progressively more peripheral rings of lamina. Consequently, the last portion represented the leaf margin. Each portion was of equal surface area $(20 \%$ of the lamina) and for each portion, the presence or absence of herbivory was noted.

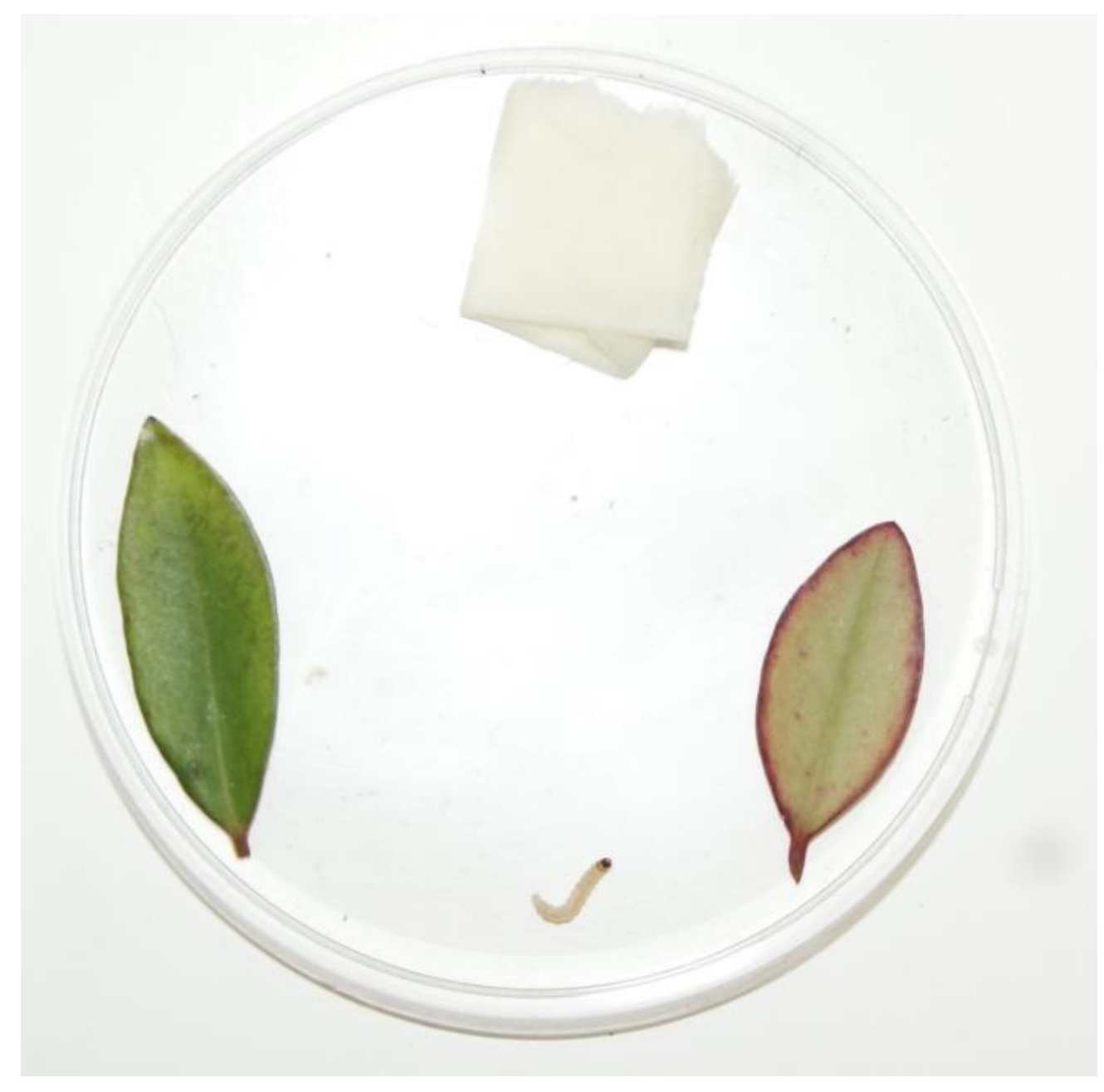

Fig 2.2. Feeding trial enclosure containing a green- (left) and red- (right) margined Pseudowintera colorata leaf and a Ctenopseustis obliquana larva. A moist paper towel (above) was used to maintain humidity. 
Approximately $24 \mathrm{~h}$ after pupation, two male and two female adult C. obliquana were introduced into each of 80 plastic pots, $18 \mathrm{~cm}$ tall x $20 \mathrm{~cm}$ diameter, which contained two cut stems of $P$. colorata held in water. The stems had been defoliated with the exception of one mature leaf, and were arranged such that each pot held one red- and one green-margined leaf. All leaves were confirmed to be free of lepidopteran eggs. To discourage oviposition on the pots themselves, their sides were lined with sandpaper, the bottoms filled with vermiculite, and the tops covered with white nylon mesh (Fig 2.3). Half of the replicates were placed under natural light in a glasshouse, and the remainder held in darkness, both areas maintained at $18{ }^{\circ} \mathrm{C}$. After $72 \mathrm{~h}$ the leaves were removed and total numbers of eggs counted.

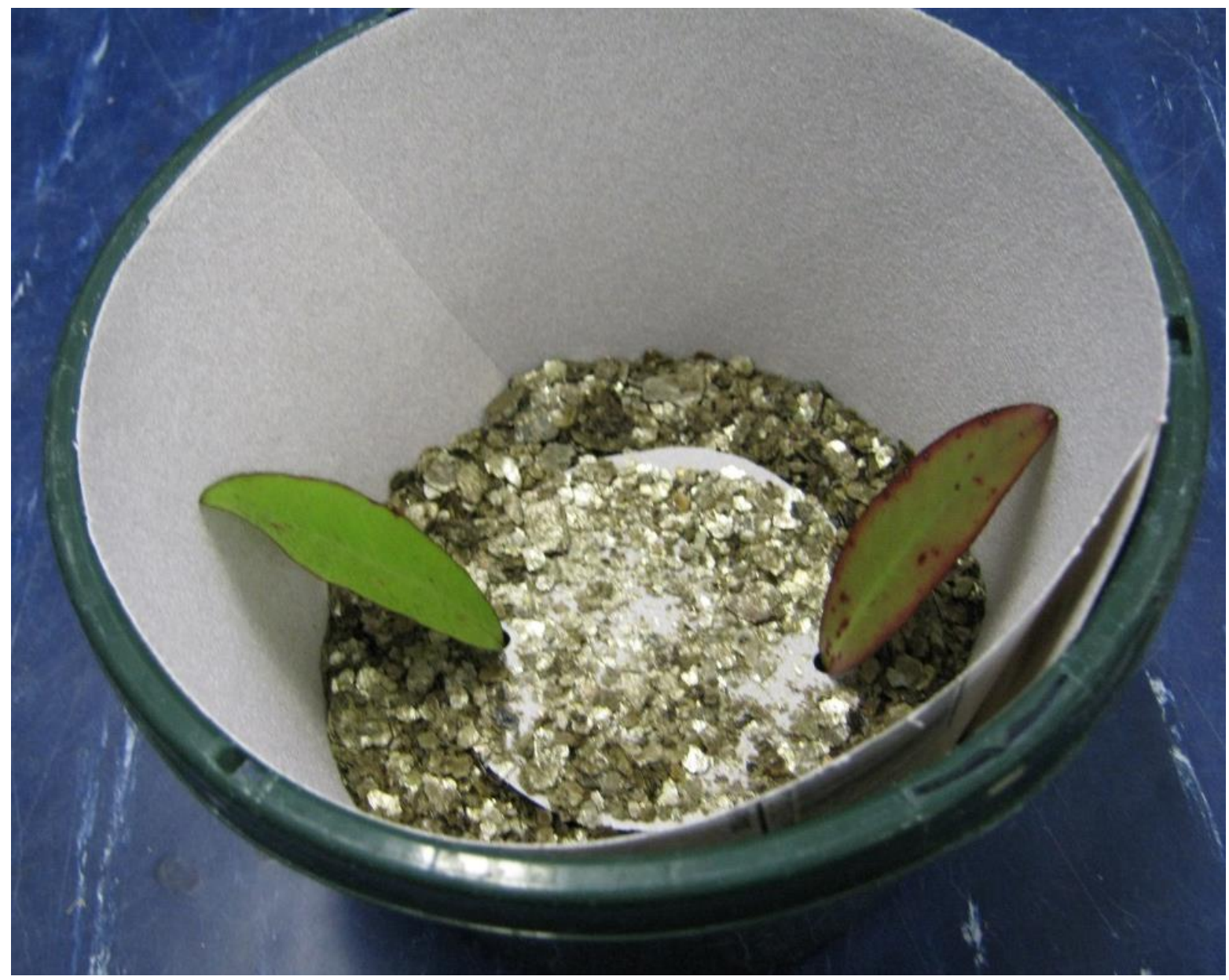

Fig 2.3. Oviposition trial enclosure containing a green- (left) and red- (right) margined leaf. Sandpaper and vermiculite were added to the sides and base of the enclosure to restrict oviposition to the leaves. 


\subsubsection{Statistical Analysis}

All statistical analyses were performed using SPSS 16.0 (Chicago, IL, USA). Margin widths and herbivory damage were $\log _{10}$ transformed to satisfy normality, and a general linear model used to evaluate their relationship, treating individuals as a random factor, and nodes as a fixed factor. Non-linear regressions using unlogged margin size and herbivory values were performed for each node. Fligner-Killeen tests were used to compare variability of area eaten for green- and red-margined leaves. To evaluate the correlation between margin width and area eaten for a whole plant, margin widths were ranked for each node from smallest to largest. Ranking margin width removed the effect of absolute margin width differences between nodes. Non-linear regressions were performed using the mean margin rank and mean area lost for each plant.

Differences in margin size between nodes, and in anthocyanin and polygodial concentrations between margin and leaf interior, were compared using paired, two-tailed t-tests. Anthocyanin and polygodial concentrations between red and green-margined leaves were compared using an independent, two-tailed $t$-test. For those leaves used in our feeding trial, the relationship between leaf colour, light treatment, and the distribution of herbivory was determined using a binary logistic regression where the presence or absence of herbivory was treated as the dependent variable, while the predictor variables were light treatment, leaf colour and the portion of lamina where herbivory occurred. Finally, a Wilcoxon signed rank test was used to compare amounts of leaf area eaten between red- and green-margined leaves following feeding trials, and numbers of eggs deposited following oviposition trials.

\subsection{Results}

\subsubsection{Pseudowintera colorata margin pigments}

The width of red leaf margins varied substantially among individuals from the forest population of $P$. colorata (Fig 2.1a-d). Margin size ranged from a barely discernible sliver to an expansive band covering up to $28 \%$ of the leaf width. The anthocyanins were predominantly located in the vacuoles of a contiguous band of epidermal cells (Fig 2.1f), which extended more than 200 cells across in some leaves. Leaves for which the margin was not visibly discernible were also found to hold small clusters of anthocyanic cells (Fig 2.1 g); these, however, extended only a few cells inwards from the margin, too small to be detected by the unaided human eye. Both the red and green regions of every leaf contained large, 
spherical idioblast oil cells, the likely sites of polygodial biosynthesis and/or storage, within the palisade and spongy mesophyll cells (Fig 2.1g).

Green leaf margins reflected substantially more light across almost the entire visible spectrum than did the red margins (Fig 2.4). However, the ratio of red $(600-700 \mathrm{~nm})$ to green $(500-600$ $\mathrm{nm})$ reflected light, was 3-fold higher in the red margins. Similarly, the ratio of blue (400-500 $\mathrm{nm}$ ) to green reflected light was almost twice as high in the red margins.

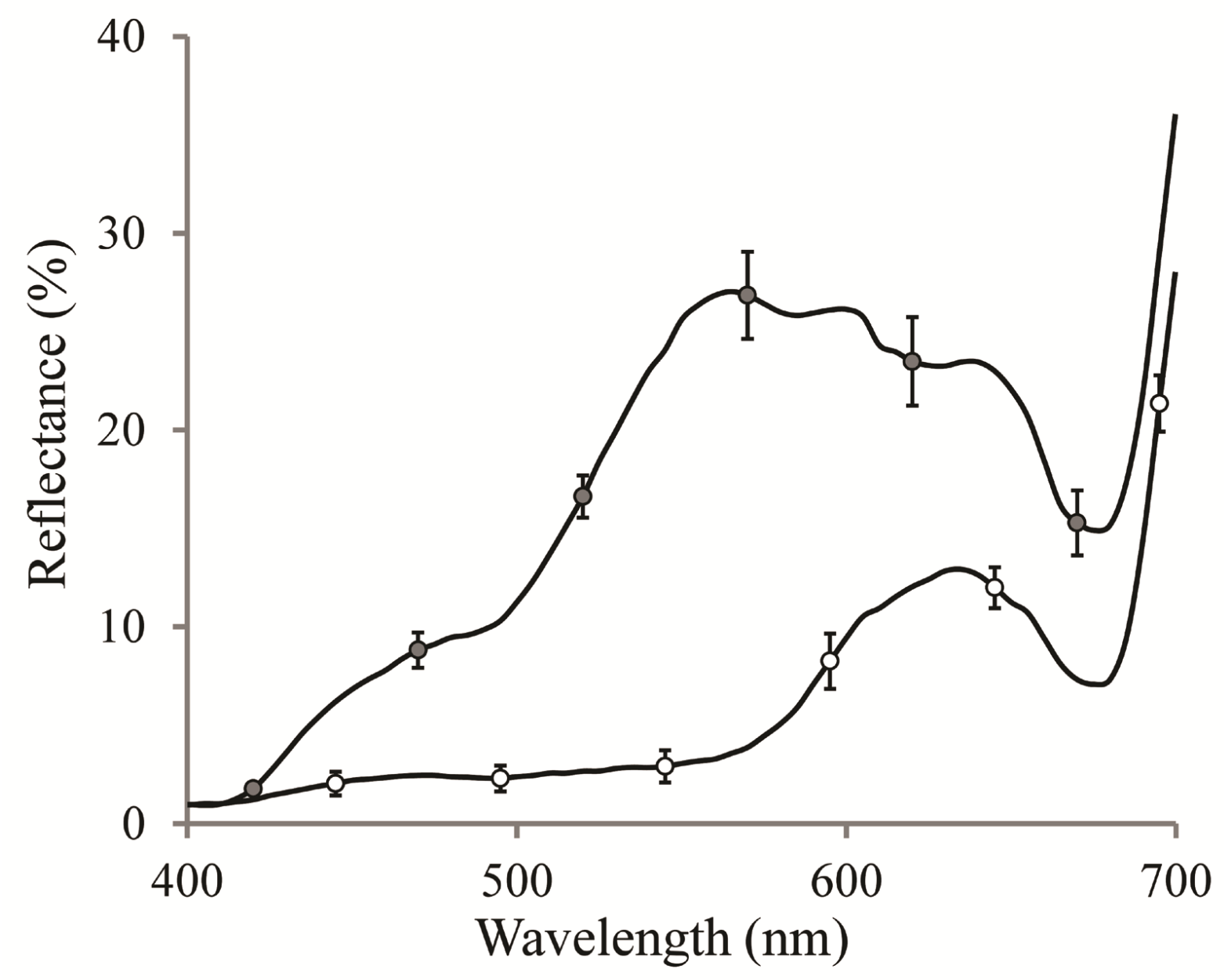

Fig 2.4. Mean $( \pm$ SE) reflectance values between $400 \mathrm{~nm}$ and $700 \mathrm{~nm}$ for the margins of red(white) and green-margined (grey) Pseudowintera colorata leaves. Symbols identify the lines; actual data points are spaced $0.4 \mathrm{~nm}$ apart; $n=5$.

Red margin width varied less among leaves within an individual than among individuals. Plants that had the wider red margins at node 1 also had wider margins at nodes 3 and 7 . Margin width at node 1 correlated positively with nodes $3\left(\mathrm{r}^{2}=0.22, P<0.001\right)$, and $7\left(\mathrm{r}^{2}=\right.$ $0.24, P=0.016$ ) for leaves on the same plant. Similarly, margin widths at node 3 correlated 
positively with margin width at node $7\left(\mathrm{r}^{2}=0.46, P<0.001\right)$. For all plants, margins were progressively narrower at consecutively older nodes $\left(F_{2,291}=40.7, P<0.001\right.$; Fig 2.5a); on average, node 3 was $40 \%$ more narrow than node 1 and node 7 was 55\% more narrow than node 7. 

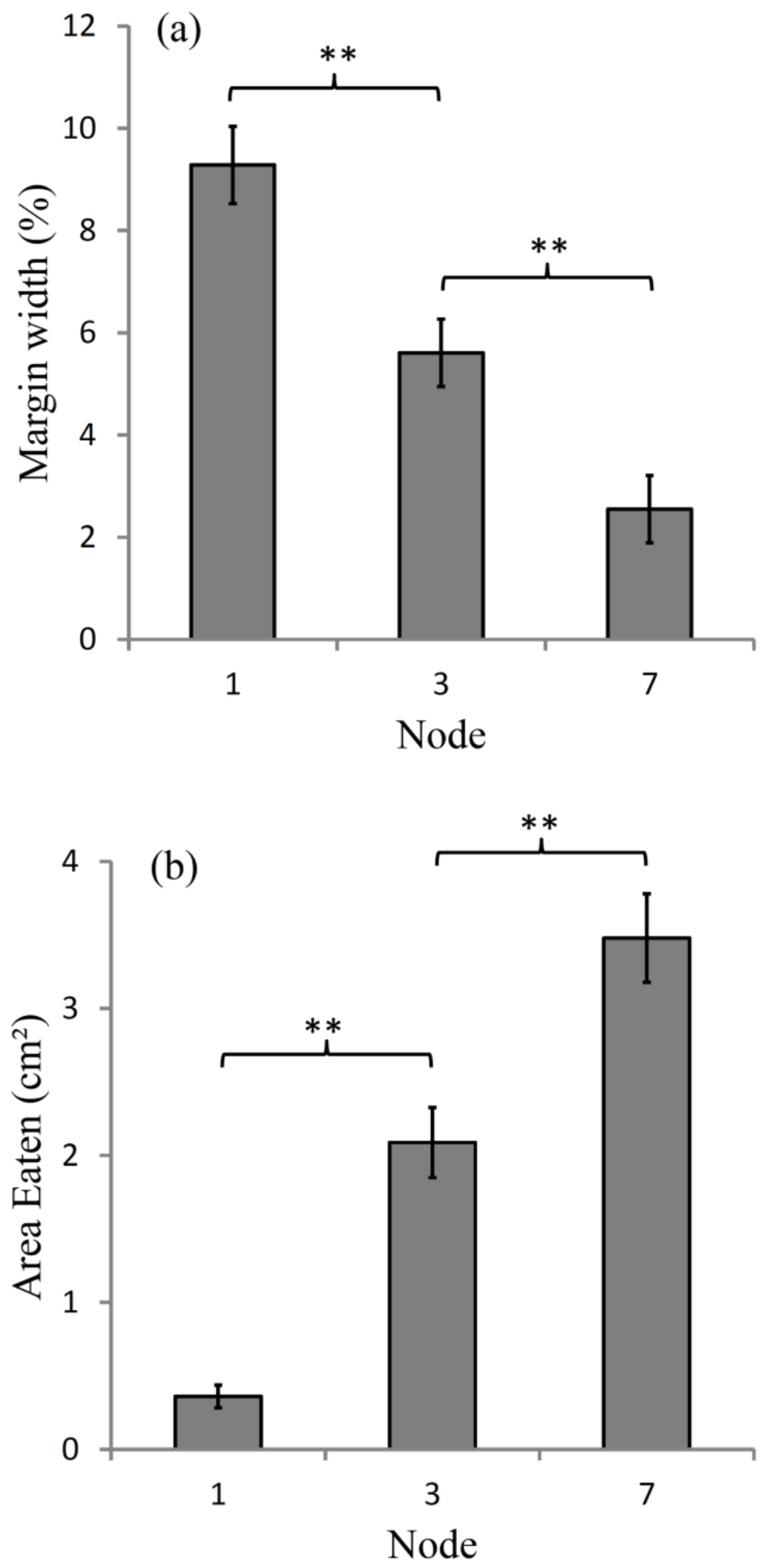

Fig 2.5. Positional differences in red margin width as percentage of lamina width (a) and area eaten (a) of Pseudowintera colorata leaves. Means $( \pm \mathrm{SE}), n=98$ leaves per node. ** Statistically significant difference between groups $(P<0.001)$. 


\subsubsection{Polygodial and anthocyanin analysis}

All leaves, regardless of margin colour, held $20 \%$ higher polygodial concentrations at the leaf margin than in the interior of the lamina (red $t_{9}=12, P<0.001$; green $t_{9}=7.2, P<$ 0.001). Compared with green leaves, those with red margins had $33 \%$ higher polygodial concentrations in the margin $\left(t_{18}=3.5, P=0.003\right)$ and $21 \%$ higher polygodial concentrations in the interior $\left(t_{18}=3.1, P=0.006\right.$; Fig 2.6a).

Anthocyanin concentrations in leaf margins exceeded those of the lamina interior for both red $\left(t_{9}=9.3, P<0.001\right)$ and green-margined leaves $\left(t_{9}=4.6, P=0.001\right)$. Compared with green leaves, those with red margins had $291 \%$ higher anthocyanin concentrations in both the margin $\left(t_{18}=6.4, P<0.001\right)$ and $150 \%$ higher concentrations in the interior $\left(t_{18}=3.8, P=\right.$ 0.001; Fig 2.6b).

Another sesquiterpene dialdehyde, 9-deoxymuzigadial, was present in trace amounts $(3 \pm 0.5$ $\mathrm{mg} \mathrm{g}^{-1}$ ) in both sets of leaves. Although 9-deoxymuzigadial has previously been shown to possess anti-feedant properties (Gerard et al., 1993), its concentration was on average 17-fold lower than that of polygodial, and so it was not considered further in this study. 

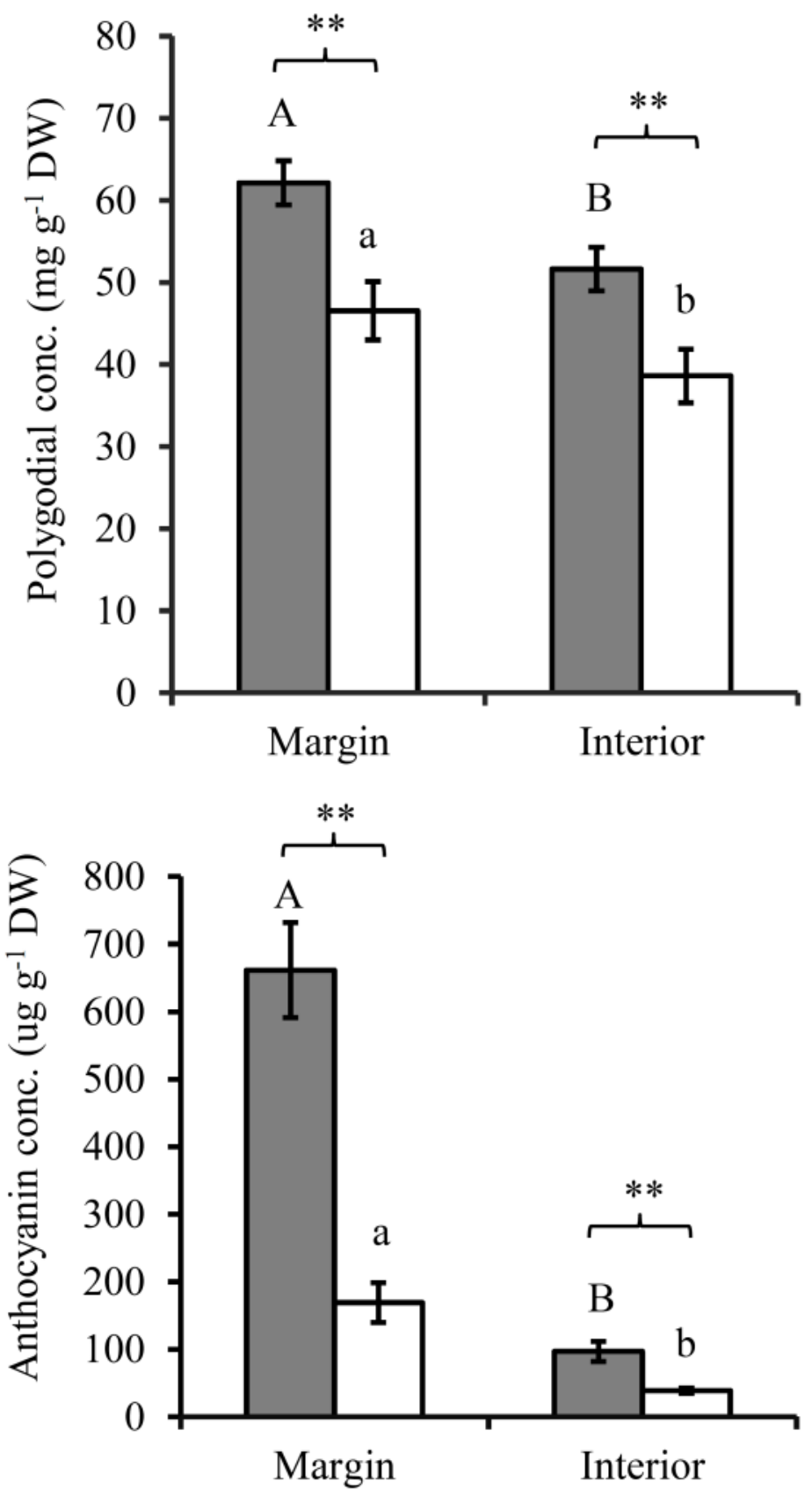

Fig 2.6. Concentrations of polygodial (a) and anthocyanins (a) in the leaf margin and interior of red- (grey bars) and green-margined (white bars) Pseudowintera colorata leaves. Means $( \pm \mathrm{SE}), n=10$. Letters above bars indicate significant difference between margin and interior $(P<0.001) .{ }^{* *}$ Statistically significant difference between leaf types $(P<0.01)$. 


\subsubsection{Herbivory}

Of the 294 leaves sampled in the field, all but 17 showed evidence of edge-feeding herbivory. The proportion of lamina consumed varied between $0.01 \%$ and $70 \%$. Although the leaves at node 1 were significantly larger $\left(17 \pm 0.5 \mathrm{~cm}^{2}\right)$ than those at nodes $3\left(15 \pm 0.7 \mathrm{~cm}^{2}\right)$ and $7(15$ $\pm 0.6 \mathrm{~cm}^{2}$ ), they had incurred $82 \%$ less edge-feeding herbivory than leaves at node 3 and $90 \%$ less herbivory than leaves at node $7\left(F_{2,291}=47.8, P<0.001\right.$; Fig $\left.2.5 b\right)$. Only leaves at node 1 showed any recent herbivory, as evidenced by the lack of necrotic tissue and anthocyanin pigmentation around wounded areas (Gould et al., 2002a).

Overall, leaves with the widest red margins had incurred the least edge herbivory (GLM, $\left.F_{1,191}=5.2, P=0.02\right)$. However, the relationship between herbivory damage and percent margin width (Fig $2.7 \mathrm{a}-\mathrm{c})$ differed significantly across the three nodes examined $\left(F_{2,191}=\right.$ 3.491, $P=0.03$ ). Consumed lamina area negatively correlated with $\%$ margin width at nodes $3\left(\mathrm{r}^{2}=0.21, P<0.001\right)$ and $7\left(\mathrm{r}^{2}=0.06, P=0.015\right)$, but not at node $1\left(\mathrm{r}^{2}=0.01 P=0.3\right)$. The relationships were non-linear, best approximated by negative logarithmic curves. In all instances, edge herbivory better correlated to the proportionate margin widths than to the absolute margin widths. When all three leaves were averaged per plant, those individuals with the largest average margin width had experienced the least herbivory. The mean rank of margin width negatively correlated with the mean area lost $\left(\mathrm{r}^{2}=0.098, P=0.002\right)$. When only nodes 3 and 7 were averaged, where most herbivory had occurred (Fig 2.5b), the relationship between rank of margin width and area lost was stronger $\left(\mathrm{r}^{2}=0.17, P<0.001\right)$.

For the leaves at nodes 3 and 7, wider red margins were also associated with a reduced variance in lamina area consumed (Fig 2.7b, c). There were critical margin widths above which variance in herbivory damage was significantly diminished. These were $1.75 \%$ for the leaves at node 3 (Fligner-Killeen, d.f. $=1, P<0.01$ ), and $2.25 \%$ for those at node 7 (d.f. $=1$, $P=0.03$ ). 

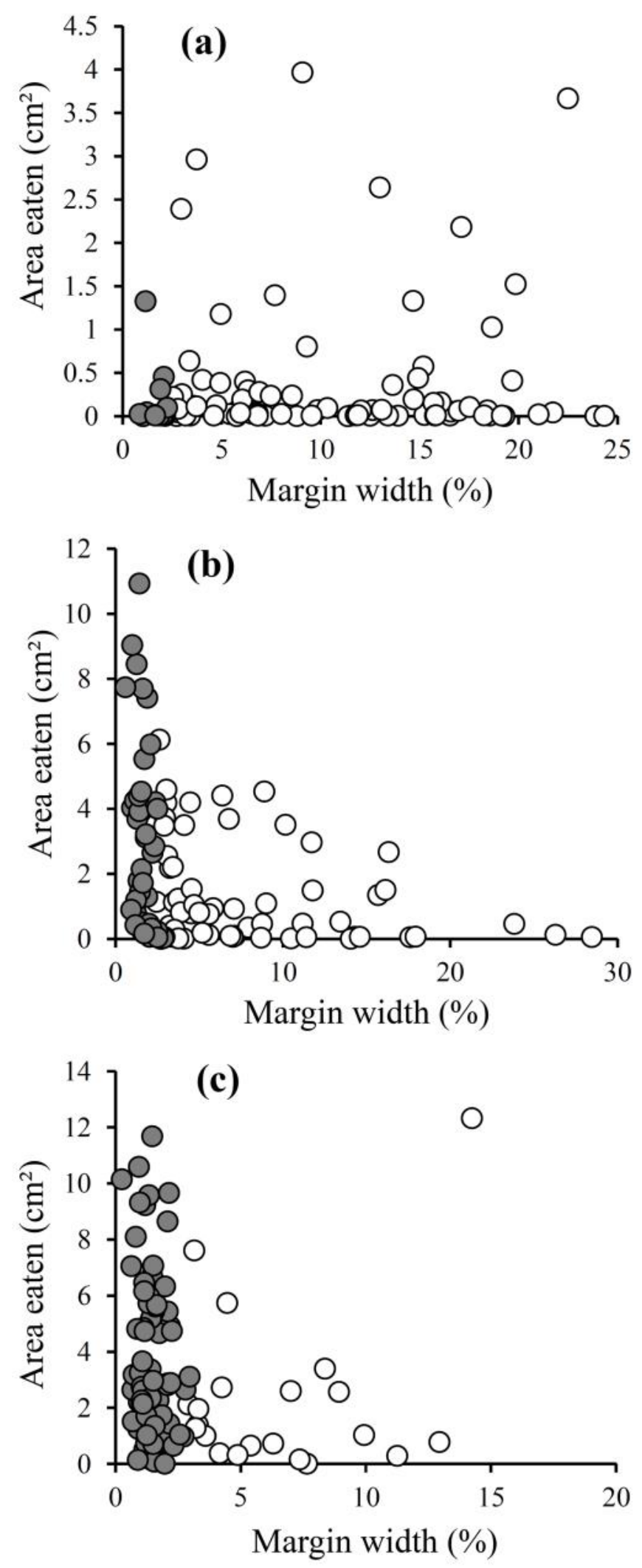

Fig 2.7. Area eaten and relative margin width of Pseudowintera colorata leaves from nodes 1 (a), 3 (b) and 7 (c). Closed symbols show points beneath the critical margin width at which variance in area consumed is significantly diminished $(P<0.05) . n=98$. 


\subsubsection{Feeding and oviposition trials}

Herbivory rates were high in the laboratory feeding trials, with only one Ctenopseustis obliquana larva out of 136 not consuming any leaf material. A further three larvae died, which reduced the numbers of replicates for white light, green light and dark treatments to 33 each. The presence or absence of herbivory was comparable for both leaf types (Wald $=0.3$, d.f. $=1, P=0.6$ ), and for leaves under all light treatments (Wald $=2.6$, d.f. $=3 ; P=0.5$ ), yet differed between portions of a leaf's lamina (Wald $=18.8$, d.f. $=4, P=0.001$ ). Herbivory occurred more frequently at the leaf margin with $85 \%$ of leaves experiencing herbivory at this position, compared to inner lamina where only $47 \%$ of leaves experienced herbivory (Fig 2.8). However, while the initiation of herbivory did not differ between leaf types or light treatments, the extent of herbivory did. Under white light, the leaf area consumed was $130 \%$ greater for the green margined leaves than for the red ones $(n=33, P=0.045$; Fig 2.9). No difference was found under green light $(n=33, P=0.3)$, red light $(n=33, P=0.5)$ or in the dark ( $n=33, P=0.6)$. Most larvae (72\% of all replicates) had consumed portions of both the red- and green-margined leaves supplied to them. For those larvae that had sampled both leaves under white light, $180 \%$ more leaf area was consumed on green- than red-margined leaves $(n=20, P=0.03)$. For the remaining replicates under white light, for which only one leaf type was chosen, no difference was found in area eaten between red- and green-margined leaves $(n=13, P=0.6)$. No difference in area eaten between red- and green- margined leaves was found for larvae that had sampled both leaf types under green light $(n=24, P=0.6)$, red light $(n=25, P=0.5)$ or in the dark $(n=27, P=0.2)$. 


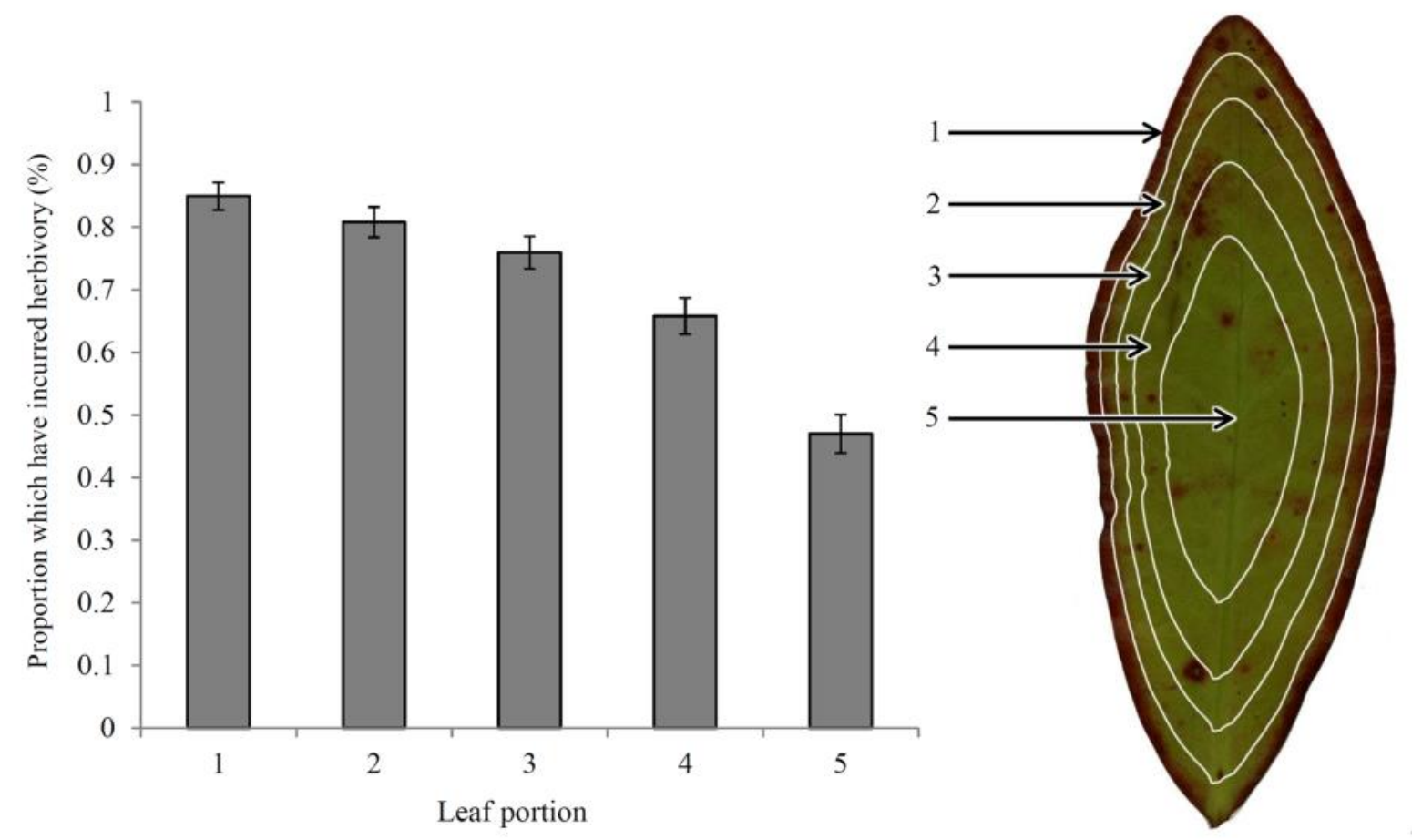

Fig 2.8. The distribution of herbivory within Pseudowintera colorata leaves. For any given leaf portion, the column represents the proportion $( \pm \mathrm{SE})$ showing herbivore damage. $n=226$. 


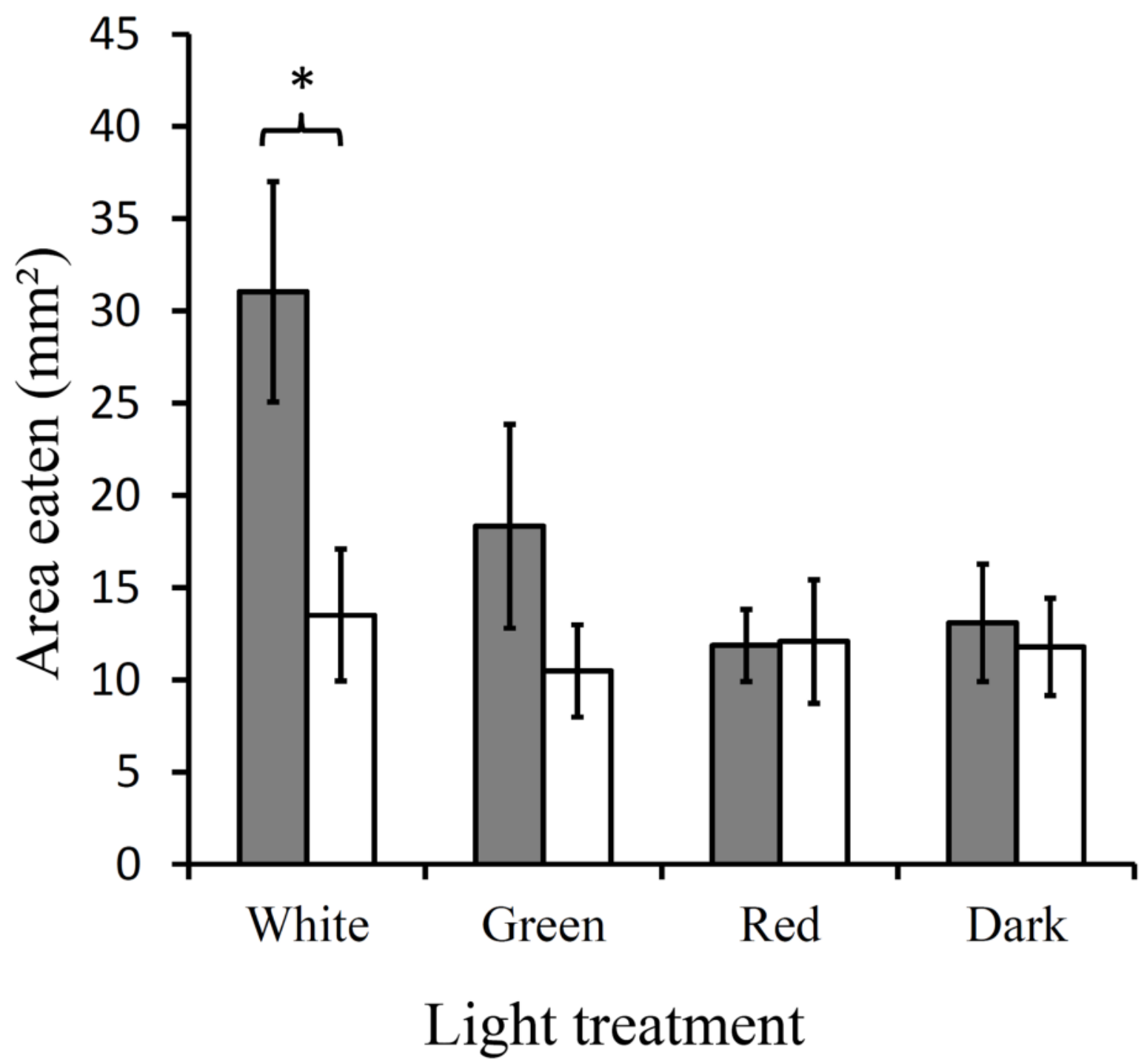

Fig 2.9. Area of red- (white bars) and green-margined (grey bars) Pseudowintera colorata leaves eaten by Ctenopseustis obliquana larvae under white, green, or red light, or darkness. Means $( \pm \mathrm{SE}), \mathrm{n}=33$. $*$ Statistically significant differences between groups $(\mathrm{P}<0.05)$.

Rates of successful egg-laying in the oviposition trials were generally low. Eggs were found in only $50 \%$ of the replicates where moths oviposited in natural light and $70 \%$ of the replicates where oviposition was completed in the dark. There was no statistical difference between oviposition rates on green- or red-margined leaves either in the light $(n=20, P=$ 0.3 ) or the dark $(n=28, P=0.2)$ environments (Table 2.1). There was also no statistical difference between oviposition rates on the adaxial and abaxial surfaces. 
Table 2.1. Mean $( \pm \mathrm{SE})$ numbers of Ctenopseustis obliquana eggs oviposited on green- and red-margined Pseudowintera colorata leaves. $n=40$.

\begin{tabular}{llll}
\hline Treatment & Position & Green-margined leaf & Red-margined leaf \\
\hline Light & Adaxial & $6(2)$ & $12(4)$ \\
& Abaxial & $2(2)$ & $10(7)$ \\
& Total & $8(3)$ & $22(9)$ \\
& Adaxial & $9(3)$ & $12(6)$ \\
& Abaxial & $5(3)$ & $2(1)$ \\
& Total & $14(4)$ & $13(6)$ \\
& & & \\
\hline
\end{tabular}

\subsection{Discussion}

Three sets of evidence from this analysis of $P$. colorata indicate that red leaf margins may function as a visual signal to deter insect herbivores. First, relative to the green leaves, those leaves that bore a wide red margin were significantly richer in polygodial, a potent insect anti-feedant (Fig 2.6a). The red margins are, therefore, a reliable signal of increased investment in this defensive compound. Second, the presence of red margins was associated with a reduced propensity for herbivory damage in a natural $P$. colorata population $($ Fig $2.7 \mathrm{~b}$, c). Third, in laboratory trials, the larvae of C. obliquana, a natural herbivore of P. colorata, preferred to feed on green- rather than red-margined leaves, but only when the experiment was conducted under white light which enabled colour vision (Fig 2.9). The larvae evidently respond to a visual rather than an olfactory signal. Collectively, these data present a compelling case for a new functional role of anthocyanins in leaf margins, and they add empirical support for the co-evolution hypothesis (Archetti, 2000; Hamilton \& Brown, 2001) 
and aposematic colouration in red leaves (Lev-Yadun, 2006; Lev-Yadun \& Gould, 2007; Lev-Yadun, 2009a; Lev-Yadun \& Gould, 2009; Lev-Yadun \& Holopainen, 2009).

\subsubsection{Defensive investment is concentrated at the margin}

Irrespective of colour, levels of polygodial were consistently greater at the leaf margins than in the leaf interior (Fig 2.6a). Previous studies have similarly shown higher concentrations of phenolic compounds (Gutterman \& Chauser-Volfson, 2000; Hughes et al., 2010b), glucosinolates (Shroff et al., 2008) and nicotine (Kester et al., 2002), and a greater trichome density (Rautio et al., 2002), all implicated in herbivory defence, at the leaf margin. This study additionally showed that herbivory pressure was greatest at the leaf margin, and that irrespective of leaf colour, a natural P. colorata herbivore, Ctenopseustis obliquana, was more likely to initiate feeding at the outer portions of a leaf (Fig 2.8). By concentrating defensive compounds around the leaf margin, plants may be selectively targeting edgefeeding herbivores. Moreover, since the interiors of red-margined leaves were also richer in polygodial than those of green leaves (Fig 2.6a), anthocyanins may indicate not only that the leaf periphery is especially rich in polygodial, but also indicate overall differences in investment in defences among individuals.

\subsubsection{Insect vision and anthocyanins}

A conceptual problem of the co-evolution hypothesis is that many insects are incapable of perceiving red hues (Doring \& Chittka, 2007; Archetti et al., 2009). While the receptors needed for perceiving red light have evolved multiple times in Lepidoptera (Briscoe \& Chittka, 2001), it is not known whether they are present in C. obliquana larvae. Nonetheless, insects lacking red receptors have been shown to distinguish red from green by using the ratio of green to blue light reflected from a leaf (Doring et al., 2009), and for P. colorata, the ratio of reflected green: blue light was almost two-fold lower in the green margins (Fig 2.4). Aphids lack red receptors yet apparently discriminate between stimuli of various colours (Doring et al., 2009). Moreover, lepidopteran larvae, including one species within the Tortricidae, the family containing $C$. obliquana, have similarly been shown to discriminate between red and green stimuli (Harris et al., 1995; Singh \& Saxena, 2004).

\subsubsection{Herbivore behaviour and leaf colour}

Compared to chemoreception, the importance of visual detection in locating insect host plants has been largely neglected (Reeves, 2011). In this study, C. obliquana larvae did not appear 
to distinguish between leaves with different margin colours when illuminated under monochromatic green or red light, or in the absence of light. However, under white light, green-margined leaves were clearly preferred (Fig 2.9). Under the monochromatic light the colour contrast between red margin and leaf interior disappears, though an achromatic contrast remains between the part reflecting light (e.g., red margin under red light) and the leaf part not reflecting light (e.g., green interior). These data indicate that the larvae used chromatic rather than achromatic contrast to distinguish between the two leaf types.

This chapter has been published (Cooney et al., 2012) and the experimental design employed in the feeding trial has subsequently been replicated. Using red and green apple (Malus domestica) leaves and larvae of the light brown apple moth (Epiphyas postvittana), Markwick et al. (2013) similarly compared herbivory preference under both light and dark conditions. They found E. postvittana larvae significantly preferred green leaves compared to red when both are presented under white light, yet when presented in the dark, this preference disappeared. Like C. obliquana, E. postvittana evidently uses leaf colour to discriminate between leaf types.

In my study, the likelihood that herbivory be initiated on a given leaf was comparable for both leaf types, regardless of light treatment. Moreover, the feeding preference for green leaves under white light was evident only when both leaf types had been sampled (see section 2.4.4). Avoidance of red margined leaves appears not to be innate but, rather, relies on gustatory or post-ingestive feedbacks i.e. larvae that sample both leaves might be associating leaf preference with leaf colour and altering their behaviour accordingly. Importantly, $C$. obliquana larvae did not use olfactory cues or the position of leaves within an enclosure to discriminate between the food sources. While previous studies have demonstrated an innate preference by some lepidopteran larvae towards green stimuli (Singh \& Saxena, 2004; Hora et al., 2005; Yasui et al., 2006), this study suggests that learning based upon colour contrasts may play an important role in distinguishing host quality.

Red leaf margins would be the more effective herbivore deterrent if the signal were perceived by gravid females as well as their larvae. Lepidopteran adults are generally more mobile than larvae (Hagstrum \& Subramanyam, 2010), and C. obliquana females deposit multiple eggs during oviposition. Contrary to my hypothesis, the frequency of oviposition by gravid $C$. obliquana was similar on green- and red-margined leaves, both under white light and in darkness (Table 2.1). Qiu et al. (1998) found that polygodial inhibited oviposition by the 
diamondback moth Plutella xylostella, though this involved an assay in which polygodial solution had been applied to the surface of test filter paper discs. My result may be attributable to the life history of the animals used for oviposition trials, for which the adults had been raised on a general purpose diet (Singh, 1983) and had no previous experience of $P$. colorata. The oviposition preference of some Lepidoptera has been shown to be influenced by their diet during larval stages (Anderson et al., 1995; Akhtar \& Isman, 2003; Chow et al., 2005; Hora et al., 2005; Olsson et al., 2006). Since the feeding preference of C. obliquana larvae appears to be a learned response, it is reasonable to hypothesise that adult oviposition preference, too, requires previous experience with $P$. colorata.

\subsubsection{Patterns of P. colorata herbivory}

For convenience, leaf margins were categorised as red or green. In reality, however, red margin size represented a continuum (Fig 2.1); clusters of anthocyanic cells were present in all $P$. colorata leaves examined, including those which, to the human eye, appeared green (Fig 2.1g). Since leaves with the larger margins incurred proportionately less damage by herbivores (Fig 2.7b, c) it is of interest, therefore, to ask if there is a critical size beneath which a red leaf margin ceases to be effective as a visual cue. We observed a sharp decline in the variance of herbivory damage when the red margin was greater than $\sim 2 \%$ of the lamina width. Thus, only an extremely small proportion of the lamina is required to be red for it to act as an effective deterrent. $P$. colorata leaves can have red margins that extend $25 \%$ or more across the leaf lamina (Fig 2.7), but the potential additional anti-herbivory benefit of these large margins appears slight.

Herbivory damage correlated more strongly with the relative than the absolute widths of leaf margins. Insect preference is evidently determined by the ratio of red to green leaf areas, rather than by margin size per se. Relative size may be easier for an insect to evaluate against a concept of the maximally acceptable margin size. Alternatively, given that margin polygodial concentrations exceeded those at the interior for all leaves, the relative size of red margins may represent the proportion of lamina that is highly defended, i.e., the ratio of highly defended lamina relative to less defended lamina.

Unlike older leaves, margin width at node 1 did not correlate with extent of herbivory (Fig 2.7a). Leaves at this position possessed the broadest margins (Fig 2.5a) and, by virtue of their location at the periphery of the canopy, were the most prominent to any approaching herbivore. However, levels of herbivory on leaves at node 1 were significantly lower than 
those at lower nodes (Fig 2.5b), most likely because these younger leaves had been exposed to herbivores for a shorter duration. Benefits of red margins would be increasingly apparent as the leaves aged.

\subsubsection{The adaptive significance of red leaf margins in $\mathrm{P}$. colorata}

Although I have referred to red margins as visual 'signals', the term has an inherent implication which is yet to be confirmed. 'Signal' implies that red margins evolved in response to herbivore pressure (Otte, 1974). The alternative, 'cue', implies that the association between red margins and polygodial evolved for another purpose and insects have subsequently altered their behaviour to make use of it. Because neither scenario can as yet be confirmed, I maintain use of 'signal' while acknowledging its implications. Despite this limitation one criterion for a signalling relationship was satisfied. The effect of a red leaf margin appears to translate to an overall benefit for an individual plant. When averaging all three nodes for a single plant, those with the largest mean rank in margin width incurred the least overall herbivory. Evidently, the benefit a single leaf receives from deterring herbivores can be scaled up to benefiting an entire plant, which is consistent with red leaf margins in $P$. colorata having evolved in response to edge-feeding herbivores.

\subsection{Conclusions}

Red leaf margins in P. colorata signal increased polygodial concentrations and correlate to reduced herbivory by edge-feeding insects. In laboratory feeding trials, a preference for green-margined leaves by $C$. obliquana was dependent on ambient light quality. When the conditions necessary to perceive margin colour were removed, no difference was found in the consumption of red- versus green-margined leaves. Although there has been increasing theoretical support for aposematic colouration in red leaves (Lev-Yadun, 2009a; Schaefer \& Ruxton, 2011), direct experimental evidence has thus far been lacking. This study is the first to demonstrate a function for this common pattern in leaf colouration. 


\section{Chapter 3: Anthocyanins perform a photoprotective function in peduncles of Sambucus nigra}

\subsection{Abstract}

Anthocyanin accumulation in floral organs has overwhelmingly been attributed to selection by animals, yet theses pigments have also been attributed to a number of physiological benefits. Here, I tested the requirements of both a physiological function, that anthocyanins provide photoprotection, and a communicative function, that anthocyanic reddening enhances frugivory, for Sambucus nigra peduncles which turn red prior to fruit maturation. I found that accumulation of red pigmentation required exposure to full sunlight and that anthocyanins reduced the quantity of green light that would normally reach chlorenchyma in the peduncle by $50 \%$. Under saturating white light, red peduncles maintained $92 \%$ higher quantum efficiencies of photosystem II compared to green peduncles, and red portions of peduncle recovered from photoinactivation $8 \%$ more than did green portions. In contrast, I found little evidence of a communicative function for peduncle reddening in $S$. nigra. Given that anthocyanin accumulation coincides with senescence in S. nigra peduncles, my data show that anthocyanins in the sterile components of reproductive organs perform a function comparable to that described for autumnal leaves.

\subsection{Introduction}

The decade long debate on the role of anthocyanins in vegetative organs has conclusively shown that these pigments are simultaneously involved in both physiological and ecological processes. It has become clear that if we are to understand the functions of anthocyanin pigments in plants, we need to give equal consideration to their effects on physiology, such as photosynthesis, as to those on plant-animal interactions. Although several authors have called for work that simultaneously addresses both physiological and communicative hypotheses for anthocyanin accumulation (Gould, 2004; Schaefer \& Wilkinson, 2004; Karageorgou \& Manetas, 2006; Schaefer \& Rolshausen, 2006), such studies are extremely rare. This situation is slightly different for anthocyanins in sterile organs on reproductive structures where physiological roles are yet to be considered. Instead, anthocyanin accumulation has exclusively been seen as a signalling adaptation to animals (Schaefer \& Braun, 2009). Nonetheless, in the absence of empirical data we cannot exclude the possibility that 
physiology, rather than signalling, has been a key driver for the evolution of these red pigments in some floral organs.

In leaves, there is evidence that anthocyanins can present a measure of protection against a variety of both abiotic and biotic stressors. For example, they may reduce the propensity for photoinhibition, or ameliorate the severity of drought, salt or heavy metal stress (ChalkerScott, 1999; Gould, 2004; Gould et al., 2009; Hatier \& Gould, 2009; Agati \& Tattini, 2010; Pollastri \& Tattini, 2011; Ferreyra et al., 2012). Equally, their non-green colouration can deter herbivory by advertising the distribution and potency of chemical defences (Archetti \& Leather, 2005; Chapter 2). In contrast, anthocyanins in the perianth, peduncle and pedicels are considered exclusively to provide visual information to pollinators and frugivores on the availability and quality of rewards (Willson \& Whelan, 1990; Schaefer, 2011; Schaefer \& Ruxton, 2011). It is far from certain, however, whether this is their only or, indeed, primary role. In apples and pears, the accumulation of anthocyanins is responsible for both, a red colour change, and a reduction in the severity of light stress (Steyn, 2009; Steyn et al., 2009). The functional hypotheses that have been repeatedly tested on leaves may similarly apply to the sterile components of reproductive organs.

Among the many putative physiological functions of anthocyanins in leaves, that of lightscreening has received the most attention. When leaves are exposed to more light than they can use for carbon assimilation, they can show a characteristic decline in photosynthetic quantum yield (Long et al., 1994; Hatier \& Gould, 2009). Prolonged photoinactivation of photosystem II can lead to the production of damaging reactive oxygen species (ROS) (Murata et al., 2007; Takahashi \& Murata, 2008; Tyystjärvi, 2008). Anthocyanins absorb a proportion of the green light that would otherwise be intercepted by chloroplasts in the lower spongy mesophyll tissues. Numerous studies have reported a reduction in the severity of photoinhibition for red leaves and stems, compared to green, when exposed to saturating light (Gould et al., 1995; Dodd et al., 1998; Feild et al., 2001; Manetas et al., 2002; Steyn et al., 2002; Gould, 2004; Hughes et al., 2005; Hatier \& Gould, 2009; Gould et al., 2010; Nielsen \& Simonsen, 2011; Hughes et al., 2014; Landi et al., 2014; Tattini et al., 2014), although exceptions have been noted (Esteban et al., 2008; Zeliou et al., 2009; Júnior et al., 2012; Liakopoulos \& Spanorigas, 2012).

Fruit maturation represents the final stage of a reproductive organ's ontogeny, and the sterile components of reproductive organs, pedicels and peduncles, abscise shortly after fruit 
removal. Those sterile organs are therefore, from a physiological point of view, senescent, just like leaves that are soon to be shed. However, no published study exploring anthocyanin function in these organs has acknowledged this. We know that for leaves, senescence represents a stage of increased susceptibility to light stress as during this time, the photosynthetic machinery is disassembled for nutrient resorption (Keskitalo et al., 2005). We also know that for many plants, leaf senescence coincides with anthocyanin accumulation which has been attributed to a photoprotective role (Feild et al., 2001; Lee, 2002; Hoch et al., 2003; Lee et al., 2003; Schaberg et al., 2003; Archetti et al., 2009; Juvany et al., 2013). Various studies have reported that anthocyanic senescing leaves display a reduction in photoinhibition, a prolonged senescence and an improved proportion of nitrogen absorption, compared to acyanic senescing leaves (Feild et al., 2001; Hoch et al., 2003; Schaberg et al., 2008; Zhang et al., 2013b), although exceptions have been noted (Manetas \& Buschmann, 2011; Misyura et al., 2012). However, it has yet to be tested whether anthocyanins impart a photoprotective benefit in the sterile components of reproductive organs that similarly accumulate anthocyanins during senescence.

Sambucus nigra (European black elder) provides us with an ideal opportunity to test whether anthocyanins impart a photoprotective benefit in peduncles. Both the intensity of reddening, and the proportion of peduncle that turns red, varies between individuals as well as between infructescences on the same plant. As one might expect were this an adaptation to avoid light stress, anthocyanin production is proportionate to light exposure and it precedes abscission, meaning, anthocyanin accumulation coincides with senescence in $S$. nigra peduncles. Conversely, peduncle reddening displays several characteristics indicative of a role in promoting frugivore visitation. Red peduncles are more chromatically contrasted against the surrounding foliage than are green peduncles, and fruits are removed more quickly by birds when peduncles are red (Schaefer \& Braun, 2009). Moreover, the extent of reddening provides information on an infructescence's reward quality, with red peduncles bearing fruits that are more sugary, and crops that are larger, compared to green peduncles. However, some characteristics of peduncle reddening suggest that a communicative role is not the primary function. Firstly, anthocyanin accumulation precedes fruit maturation, making peduncles appear 'unnecessarily' red while fruits are still unripe. Secondly, peduncles borne on shaded infructescences remain green. We know that anthocyanin accumulation is not constrained by shade as shaded fruits still turn black, which begs the question, if anthocyanins are produced to attract frugivores, why do shaded peduncles not similarly turn red? It may be that 
anthocyanin accumulation in S. nigra peduncles primarily serves a physiological function, to prevent the detrimental effects of light stress during senescence. Accordingly, its role in frugivore communication would be a by-product of this colour change.

\subsubsection{Aims and hypotheses}

In this study, I investigated both: a communicative role, and a light screening role for anthocyanins in S. nigra peduncles. To test the requirements of a communicative role I monitored fruit removal for infructescences of varying redness, and compared peduncle colour for infructescences whose crop size had been experimentally manipulated. According to my communication hypothesis, peduncle reddening would enhance fruit removal, and would be proportionate to reward quality (i.e. crop size). To test for a photoprotective role for anthocyanins in S. nigra peduncles, I hypothesised that exposure to full sunlight is the requirement for anthocyanin accumulation, that the presence of anthocyanins improves the quantum efficiency of photosynthesis under high light and that anthocyanins would be responsible for reducing photoinactivation following light stress.

Finally, if the function of peduncle anthocyanins is photoprotection, comparable to that described for senescing leaves, this function may similarly be present in leaves of S. nigra, the petioles of which similarly turn red during senescence. To test the requirements of a photoprotective function for anthocyanins in the petioles of Sambucus nigra leaves, I hypothesised that the presence of anthocyanins improves the quantum efficiency of photosynthesis under high light and reduces the level of photoinactivation following light stress.

\subsection{Materials and Methods}

\subsubsection{Study Site}

Two field sites were used in this study, one in each hemisphere, so that two fruiting seasons could be utilised within a single year. The first was a deciduous forest near Freiburg, Germany (48.0 N, 7.518 E), where Sambucus nigra flowers from May to July, and fruits until September. The second site was near Greytown, New Zealand (41.6 S, 175.3 E) in a forested area on private property. S. nigra was introduced to New Zealand from Europe at least 140 years ago, and is now a common pest species (Webb et al., 1988). In this area, S. nigra flowers from November to January, with fruiting extending to February and foliar senescence 
occurring from March onwards. At both locations S. nigra was a small tree, up to $5 \mathrm{~m}$ high, most prominent at the forest margins, and was present in both fully exposed and shaded understory locations.

The frugivore visitation, shading and sugar manipulation experiments were carried out in Germany. All other research was carried out in New Zealand.

\subsubsection{Fruit removal}

To determine whether peduncle reddening enhances avian fruit removal, I monitored removal rates from 24 infructescences of varying peduncle colour, each from a separate plant. Plants were at least $50 \mathrm{~m}$ apart and individuals of a similar size and comparable fruit crop (as estimated by observation), were preferentially selected. From each plant, an infructescence was selected at random and the numbers of ripe, unripe and dead fruits were counted. A net, $40 \mathrm{~cm}$ in diameter was hung underneath each infructescence and at least once a week, the numbers of ripe, unripe and dead fruits were again counted. After counting, dead fruits were removed. Dispersal was calculated as the number of fruits removed from a given infructescence minus the number of fruits in that infructescence's drop net. Each drop net was arranged such that no fruits from adjacent infructescences could fall in.

\subsubsection{Reward manipulation experiment}

To determine whether peduncle colour reflects the quantity of an infructescence's crop size, unripe fruits were removed while peduncles were still green, then the extent of peduncle reddening was measured after fruits had ripened. For each of 46 plants, four infructescences were selected and assigned to one of four treatments: (A) control, all fruits retained; (B) 80\% of whole fruits removed randomly across the infructescence; (C) $50 \%$ of whole fruits removed randomly across the infructescence; and (D) $50 \%$ of whole fruits removed from two of each infructescence's second-order peduncles (henceforth termed 'rays'). Infructescences were preferentially selected that were of a similar size, similar height of $1.75 \mathrm{~m}$ on the tree, similar azimuth and therefore, similar exposure to sunlight. When the remaining fruits had ripened, the infructescences were excised and two $10 \mathrm{~mm}$ long portions were removed from each peduncle and one of the rays. One portion was used for quantification of anthocyanins as described below, and from the other, total soluble sugars were estimated using an Eclipse hand-held refractometer (Bellingham \& Stanley Ltd, Tunbridge Wells, UK). Cell sap was extracted by placing the material between two plastic boards, $5 \mathrm{~mm}$ thick, and crushed 
manually using polygrip pliers. The effect of crop manipulation on fruit sugar content was also determined by refraction. For each infructescence, five random fruits were removed once ripe and fruit sugar content was calculated and averaged for each infructescence.

\subsubsection{Shading experiment}

For each of 10 plants grown in full sunlight, two infructescences were selected while fruits were immature and peduncles still green. For one infructescence from each plant, the peduncle and one ray were shaded using two sheets of Rosco Supergel (Stamford, CT, USA) neutral density polycarbonate filter (\#398), which permitted the transmittance of $84 \%$ sunlight but did not alter the spectral quality of light. The remaining infructescence was left exposed to full sunlight as a control. After ripening, infructescences were removed and from each peduncle and ray, a $10 \mathrm{~mm}$ portion was excised and anthocyanins were extracted in MeOH: $\mathrm{H}_{2} \mathrm{O}: \mathrm{HCl}$ (16:3:1 v:v:v). Samples were weighed, ground, and clarified by centrifugation, and absorbance peaks measured using an Ultrospec 3000 spectrophotometer (Pharmacia Biotech, Cambridge, UK). Anthocyanin concentrations were estimated as $\mathrm{A}_{530}$ $0.24 \mathrm{~A}_{653} \mathrm{~g}^{-1} \mathrm{FW}$ (Gould, 2000).

\subsubsection{Light transmittance through the peduncle}

To quantify the proportion of light that is absorbed by anthocyanins, portions of peduncle from five green and five red infructescences were bisected longitudinally, and then each half cut to form a longitudinal wedge tapering at $30^{\circ}$. These were mounted in water, epidermis side down, on a glass microscope slide with a coverslip on an Olympus AX70 compound microscope (Olympus Optical Co., Hamburg, Germany) to which an Ocean Optics USB4000 spectrometer (Dunedin, FL, USA) had been connected to the eyepiece via a fibre optic probe. A custom-built attachment to the ocular tube ensured that the tip of the probe was perfectly centred in the optical field at the plane of focus, and prevented the entry and exit of stray light. As described by Gould (2010), specimens were sub-illuminated with white light from a 12 V/100 W Philips 7724 quartz halogen lamp in the microscope, and transmittance, the proportion of incident light transmitted through the peduncle, was measured at $0.4 \mathrm{~nm}$ intervals from 400-700 nm using SpectraSuite spectroscopy operating software. 


\subsubsection{Rapid Light Curves}

Quantum efficiencies for photosystem II (PSII) for red and green peduncles were estimated using chlorophyll fluorescence. In February 2014, after fruits had ripened, 12 infructescences were chosen from individual plants, six bearing red peduncles, and six bearing green peduncles. Infructescences were dark-acclimated for $1 \mathrm{~h}$ and the ratio of variable to maximum fluorescence $(F v / F m)$ was measured using a Walz PAM-2500 chlorophyll fluorometer (Walz Heinz GmbH, Effeltrich, Germany) at $20{ }^{\circ} \mathrm{C}$. Each peduncle was then subjected to an irradiance ramp of six steps, each $180 \mathrm{~s}$ long, sufficient for the fluorescence yield to reach steady-state $(F s)$, with light intensity incrementally advancing from 1 to 1700 $\mu \mathrm{mol} \mathrm{m} \mathrm{m}^{-2} \mathrm{~s}^{-1}$. At each step the quantum yield of PSII $\left(\Phi_{P S I I} ;\left[F m^{\prime}-F s\right] / F m\right.$ ', where $F m^{\prime}$ is maximal fluorescence following irradiation with an actinic light source), photochemical quenching $\left(q P ;\left[F m^{\prime}-F s\right] /\left[F m^{\prime}-F O^{\prime}\right]\right.$, where $F_{O}{ }^{\prime}$ is fluorescence in the absence of an actinic light source), and non-photochemical quenching (NPQ; $\left[\mathrm{Fm}-\mathrm{Fm}{ }^{\prime}\right] / \mathrm{Fm}$ ') was measured. Such rapid light curves are described as accurate predictors of photosynthetic health (White \& Critchley, 1999; Ralph \& Gademann, 2005). To compare quantum efficiencies for red and green senescing petioles, this process was repeated in March, 2014, for one leaf bearing a red petiole, and one leaf bearing a green petiole, from each of six plants.

\subsubsection{Photoinhibitory treatments for S. nigra peduncles}

Comparative levels of photoinactivation for green and mixed red/green peduncles were derived from chlorophyll fluorescence parameters following a treatment of light stress. For each of six plants, two ripe infructescences were selected; of which one bore an entirely green peduncle, and the other a peduncle that was green at its base but red towards its apex. Infructescences were excised, peduncles dark-acclimated for $1 \mathrm{~h}$, and $F v / F m$ measured at both the basal and apical ends of all peduncles using a Walz PAM-2500. These infructescences were then given $4 \mathrm{~h}$ of $1200 \mu \mathrm{mol}$ photons $\mathrm{m}^{-2} \mathrm{~s}^{-1}$ white light provided by a bank of 12 x 1 W LEDs (Model OS9151, Shaoxing Prolux Lighting Co. Ltd., Zheijiang, China), for which the colour temperature was $6500 \mathrm{~K}$. Following this light treatment and an additional $1 \mathrm{~h}$ re-acclimation period in the dark, $F v / F m$ was again measured.

\subsubsection{Light-screening experiment}

Monochromatic light treatments were used to determine if a difference in photoinactivation was specific to those wavelengths which anthocyanins absorb. For each of six plants, two ripe 
infructescences, one bearing a green peduncle and the other a fully red peduncle, were selected. Infructescences were excised, peduncles dark-acclimated for $1 \mathrm{~h}$, and $F v / F m$ measured at the base, centre, and apex of all peduncles using a Walz PAM-2500. Entire peduncles were then irradiated with $500 \mu \mathrm{mol}$ photons $\mathrm{m}^{-2} \mathrm{~s}^{-1}$ of monochromatic red, green or blue light for $6 \mathrm{~h}$. Light was provided by a bank of 12 x 1 W LEDs (Model OS9151, Shaoxing Prolux Lighting Co. Ltd., Zheijiang, China) filtered through one of three Rosco supergel polycarbonate filters: \#389 (green; maximum transmittance 500-520 nm), \#78 (blue; 440-460nm) and \#19 (red; > $620 \mathrm{~nm}$ ). The depression in $F v / F m$ was recorded immediately following each light treatment.

\subsubsection{Photoinhibitory treatments for S. nigra petioles}

To test whether peduncle anthocyanins perform a comparable function to that of foliar anthocyanins, levels of photoinactivation for $S$. nigra petioles were similarly derived from chlorophyll fluorescence parameters following light stress. For each of 12 plants, two senescing leaves were selected, of which one bore an entirely red petiole and one bore an entirely green petiole. Leaves were excised and dark-acclimated for $1 \mathrm{~h}$, and $F v / F m$ was measured using a Walz PAM-2500. Leaves were then irradiated at $1200 \mu \mathrm{mol}$ photons $\mathrm{m}^{-2} \mathrm{~s}^{-1}$ white light provided by a bank of 12 x 1 W LEDs (Model OS9151, Shaoxing Prolux Lighting Co. Ltd., Zheijiang, China) for $4 \mathrm{~h}$; six pairs of leaves were irradiated at $21^{\circ} \mathrm{C}$ and six pairs of leaves were irradiated under an additional cold stress at $4{ }^{\circ} \mathrm{C}$. The depression in $\mathrm{Fv} / \mathrm{Fm}$ was recorded immediately following each light treatment

\subsubsection{Statistical analysis}

All statistical analyses were performed using IBM SPSS 20.0 (Chicago, IL, USA). The relationship between fruit removal and a peduncle's anthocyanin content was evaluated using a general linear model. The number of fruits dispersed was treated as the dependent variable while the predictor variables, both covariates, were the fruit crop of an infructescence and the anthocyanin content of that infructescence's peduncle. General linear models (GLM) were also used to evaluate the relationship between manipulated crop size and sugar, as well as the relationship between crop size and anthocyanin concentrations. Sugar or anthocyanin concentrations were log transformed to achieve normality, and each then treated as dependent variables. For the independent variables, individual plants were treated as a random factor and treatment as a fixed factor. For those infructescences whose crop size was not 
manipulated, a nonlinear logarithmic regression was used to evaluate the relationship between fruit sugar and peduncle anthocyanins. T-tests were used for post hoc analysis and to compare anthocyanin concentrations for shaded and exposed tissues, as well as differences in photosynthetic parameters, derived from chlorophyll fluorescence, for red and green peduncles.

\subsection{Results}

\subsubsection{Anthocyanins and light abatement}

In both the German and New Zealand populations of $S$. nigra, peduncle colour varied from entirely green to entirely red (Fig 3.1A). Reddening progressed from the pedicels towards the base of the peduncle, and the proportion of the peduncle that had turned red varied both within a plant, and between plants within each population. Those infructescences that were most exposed to sunlight were most intensely pigmented red to the human eye. Similarly, while the upper and lower side of peduncles both turned red, the upper, exposed portions were the more intensely pigmented red. The anthocyanins, previously identified from fruit of S. nigra as cyanidin 3-O-glucoside and cyanidin 3-O-sambubioside (Wu et al., 2004; Veberic et al., 2009), were located in the vacuoles of two contiguous bands of the outermost layers of hypodermal collenchyma (Fig 3.1B). The green peduncles were mostly devoid of anthocyanins (Fig 3.1C), although individual anthocyanic cells were occasionally found. 


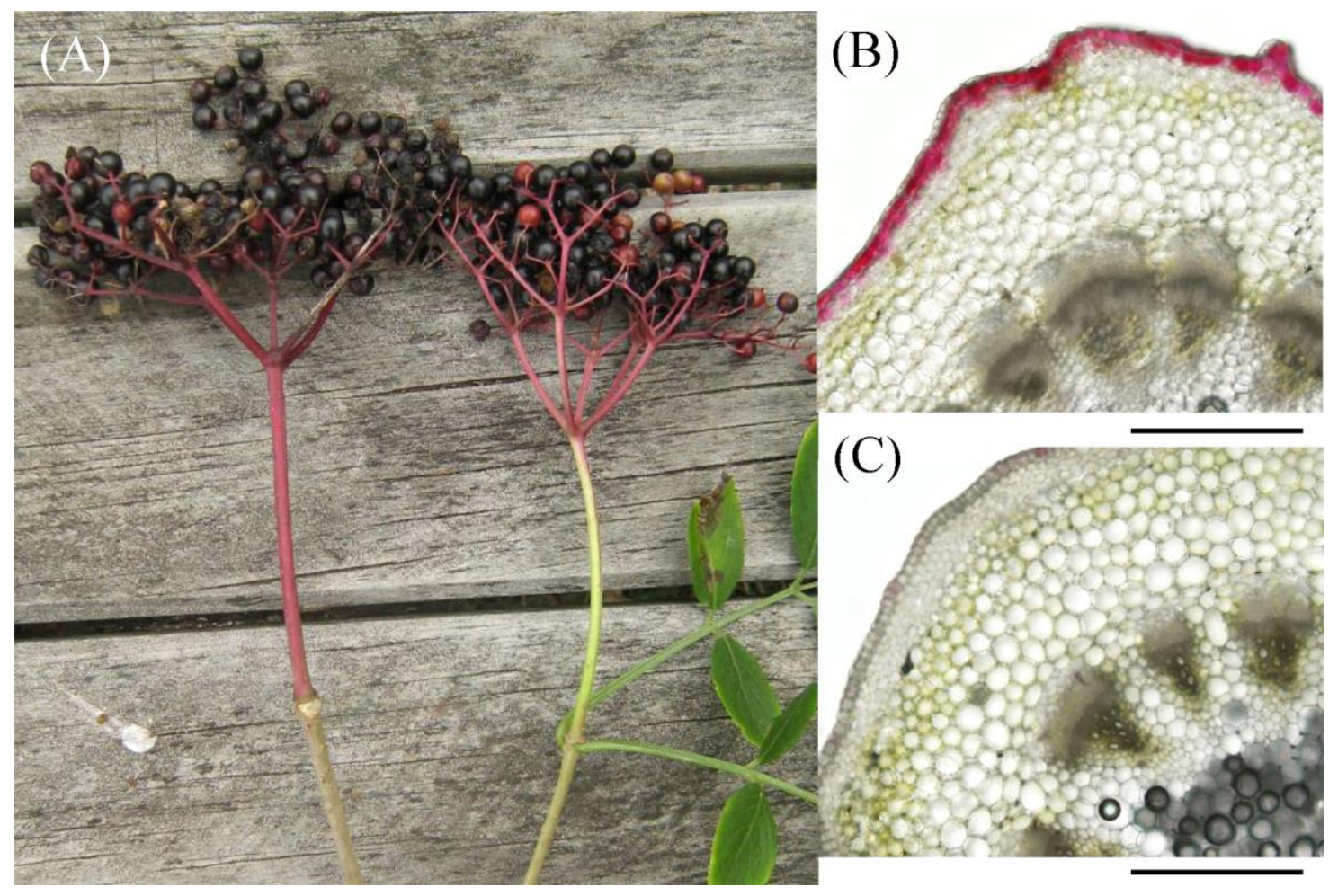

Fig 3.1. Infructescences of Sambucus nigra. Photograph of red and green peduncles (A), and photomicrographs of transverse sections through red (B) and green (C) peduncles. Bars, 500 $\mu \mathrm{m}$.

The presence of sub-epidermal anthocyanins in S. nigra peduncles significantly reduced the quantity of green/ yellow light $(485-600 \mathrm{~nm})$ transmitted to subjacent chlorencyma (Fig 3.2A; $P<0.05)$. Transmission of blue wavelengths $(400-500 \mathrm{~nm})$ at all tissue depths was comparable for red and green peduncles (Fig 3.2B). For green wavelengths, transmittance was reduced at tissue depths up to $300 \mu \mathrm{m}$ (Fig 3.2C; $P<0.05$ ) for red peduncles relative to the green, and this was most pronounced at $40 \mu \mathrm{m}$, the cell depth subjacent to sub-epidermal anthocyanic cells. At this depth the amount green light transmitted through red peduncles was half that of green peduncles (Fig 3.2C). For red wavelengths $(600-700 \mathrm{~nm})$, transmittance was comparable at all tissue depths for red and green peduncles (Fig 3.2D) 

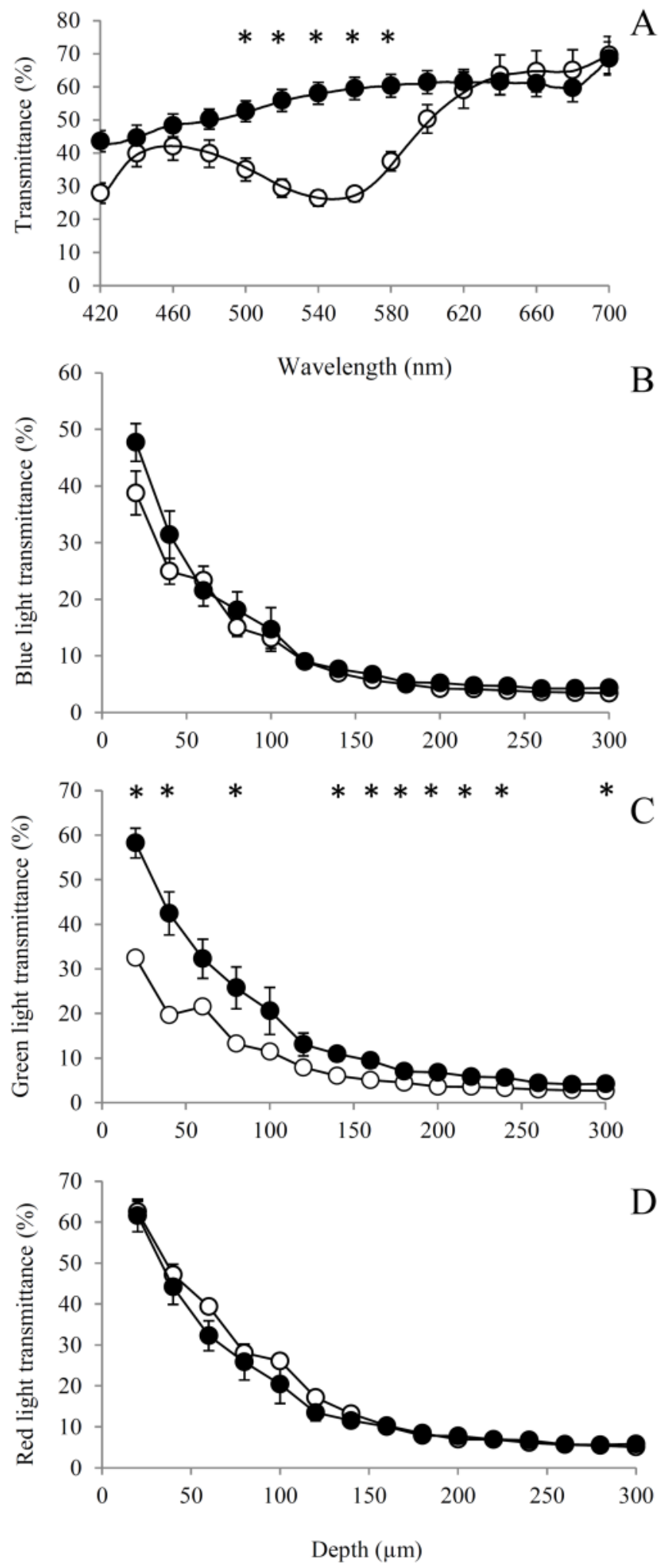

Fig 3.2. Light transmittance through peduncles of Sambucus nigra. (A) Spectral profile of transmitted light at a tissue depth of $40 \mu \mathrm{m}$. Profiles of (B) blue, (C) green and (D) red light transmitted at progressive depths through red $(O)$ and green $(O)$ peduncles. Means $( \pm S E), n$ $=5$. Statistically significant differences between peduncle types: $*, \mathrm{P}<0.05$. 
For leaves of $S$. nigra, reddening occurred in the petiole, rachis, and the basipetal section of midrib within the leaflets. Like that pattern described for peduncles, petiole reddening was exclusive to those petioles in high-light environments, while those leaves in shaded areas remained green (Fig 3.3A-B). Similarly, anthocyanic cells formed a contiguous band in the petiole, rachis and leaflet midrib, however it was absent from all lamina tissue examined (Fig 3.3C-D).

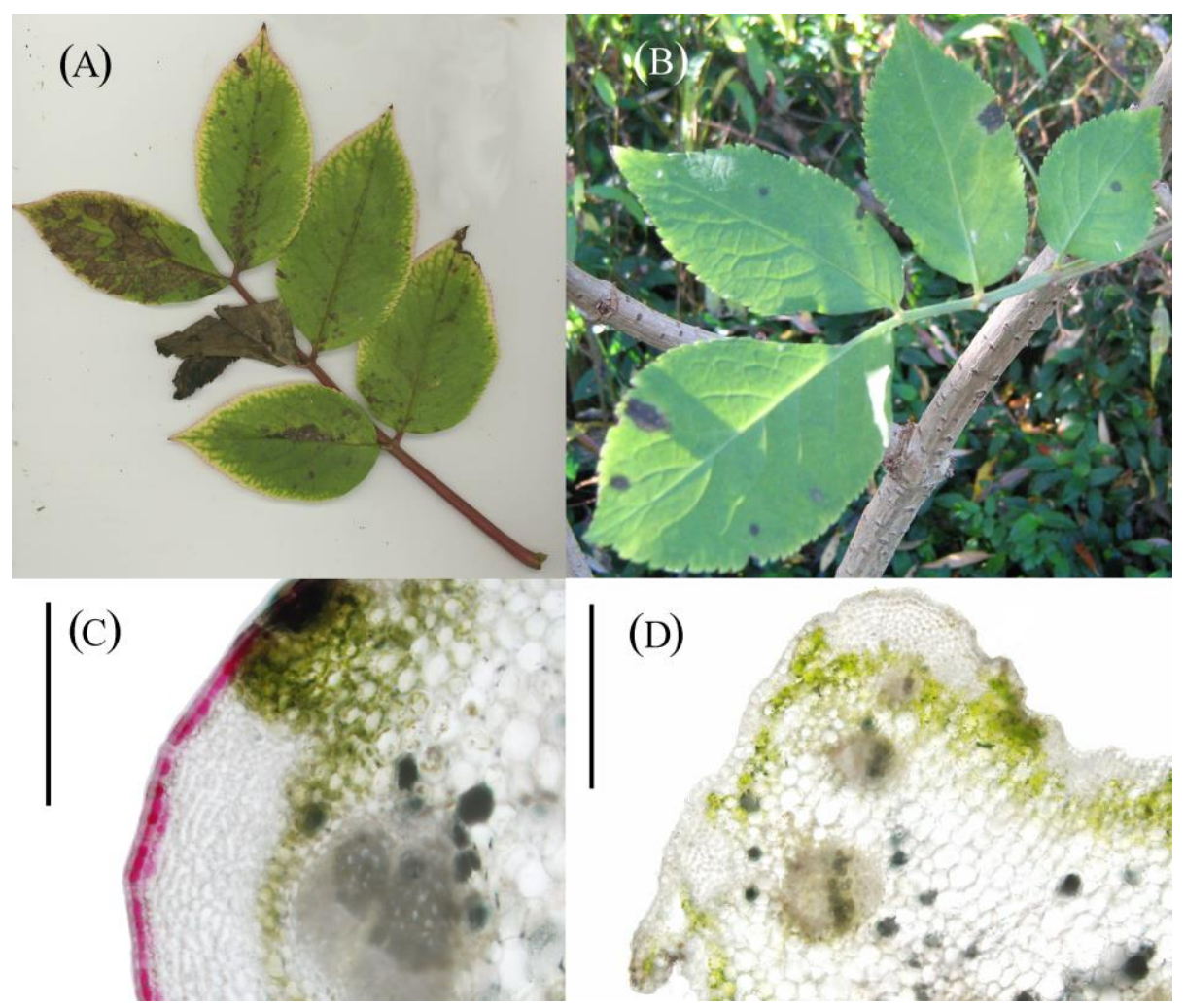

Fig 3.3. Photograph of (A) red and (B) green Sambucus nigra petioles and photomicrographs of transverse sections of (C) red and (D) green Sambucus nigra petioles.

\subsubsection{Frugivory}

Among the 24 infructescences from which fruit removal was monitored, anthocyanin concentrations in the peduncle, the number of fruits dispersed, and the proportion of fruits dispersed, varied considerably. For some plants, no fruits were eaten, while for others, up to $85 \%$ of fruits were removed. However, anthocyanin concentrations in the peduncle or the ray did not correlate with either the total numbers of fruits dispersed $(P=0.7 \& P=0.4$ respectively), or the proportion of fruits dispersed $(P=0.9 \& P=0.5$ respectively). 


\subsubsection{Manipulation of reward quality}

For those infructescences from which no fruits were removed, peduncle and ray anthocyanin content provided a reliable indication of fruit sugar content $\left(r^{2}=0.1 ; P=0.04 \& r^{2}=0.24 ; P\right.$ $=0.001$ respectively). Those infructescences for which the peduncles and rays contained the highest concentrations of anthocyanins, also bore fruits with the highest sugar content (Fig 3.4). Experimental manipulation of fruit numbers did not have any effect on anthocyanin concentrations in either the peduncle or ray $\left(F_{3,135}=1 ; P=0.4 \& F_{3,135}=0.4 ; P=0.8\right.$ respectively), but it did affect fruit sugar content $\left(F_{3,135}=7 ; P<0.001\right)$. This difference was evident amongst those infructescences from which $50 \%$ of fruits were removed from two of the infructescence's four rays, for which fruit sugar was significantly lower compared to all other treatments $(P<0.01$; Fig 3.5). Reducing the quality of an infructescence's reward, either by a reduction in crop size, or a reduction in fruit sugar, evidently had no effect on anthocyanin concentrations (Fig 3.5).

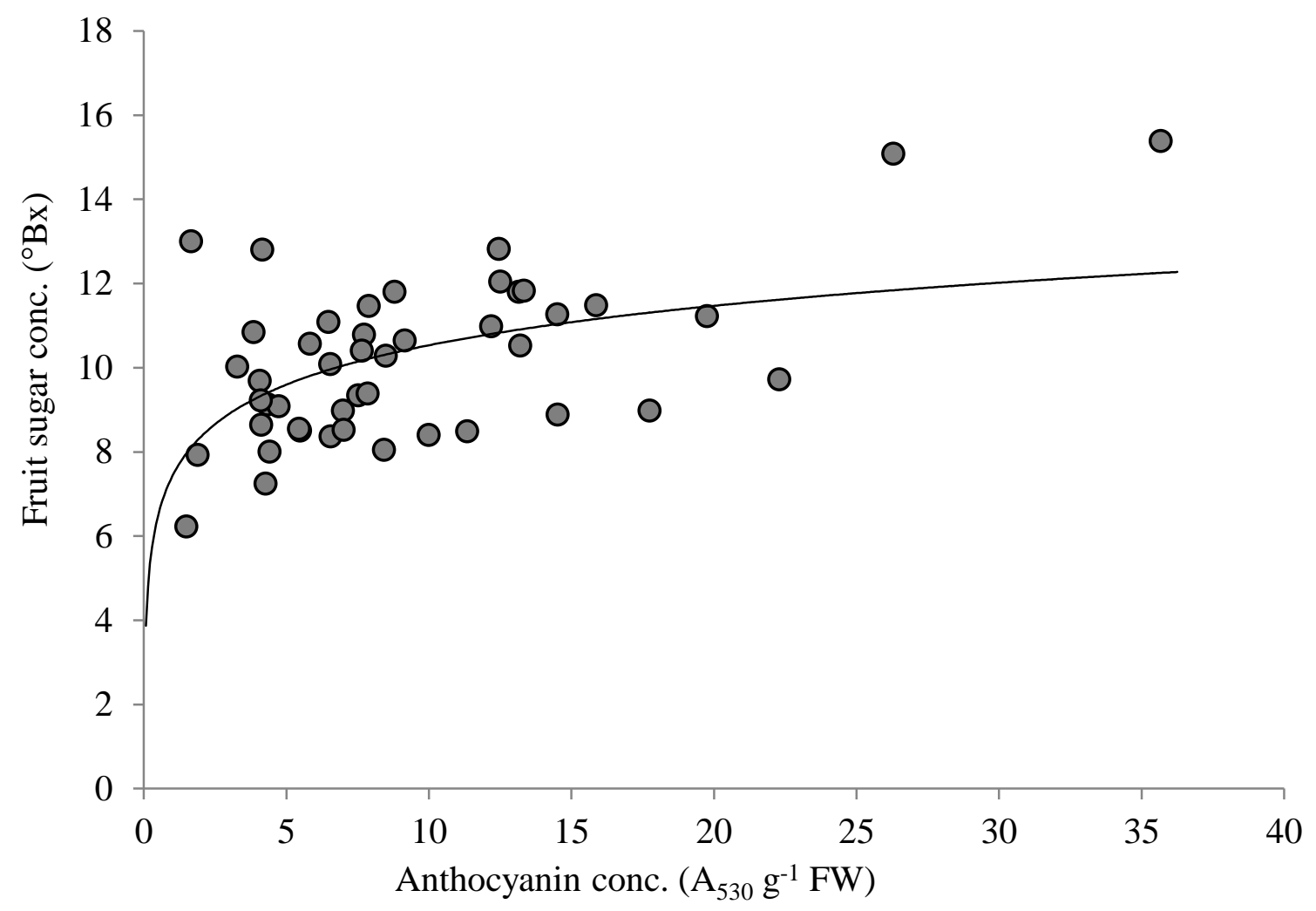

Fig 3.4. Fruit sugar concentration (degrees Brix) and ray anthocyanin concentration for Sambucus nigra infructescences. $r^{2}=0.24, n=46$. 
As well as affecting fruit sugar, manipulating the numbers of unripe fruits on an infructescence also had a significant effect on sugar concentrations in the peduncle and pedicel after fruit maturation $\left(F_{3,135}=10 ; P, 0.001 \& F_{3,135}=9 ; P=0.001\right.$ respectively $)$, though only for one treatment. Removing $80 \%$ of the unripe fruits from an infructescence during development significantly decreased sugar concentrations in both the ray and peduncle after fruits had ripened (Fig 3.5). For those treatments from which 50\% of an infructescence's fruits had been removed, peduncle and ray sugar concentrations were comparable to those in control infructescences from which no fruits were removed, irrespective of whether the fruits were removed randomly from the entire infructescence, or were removed exclusively from two of the four rays. 

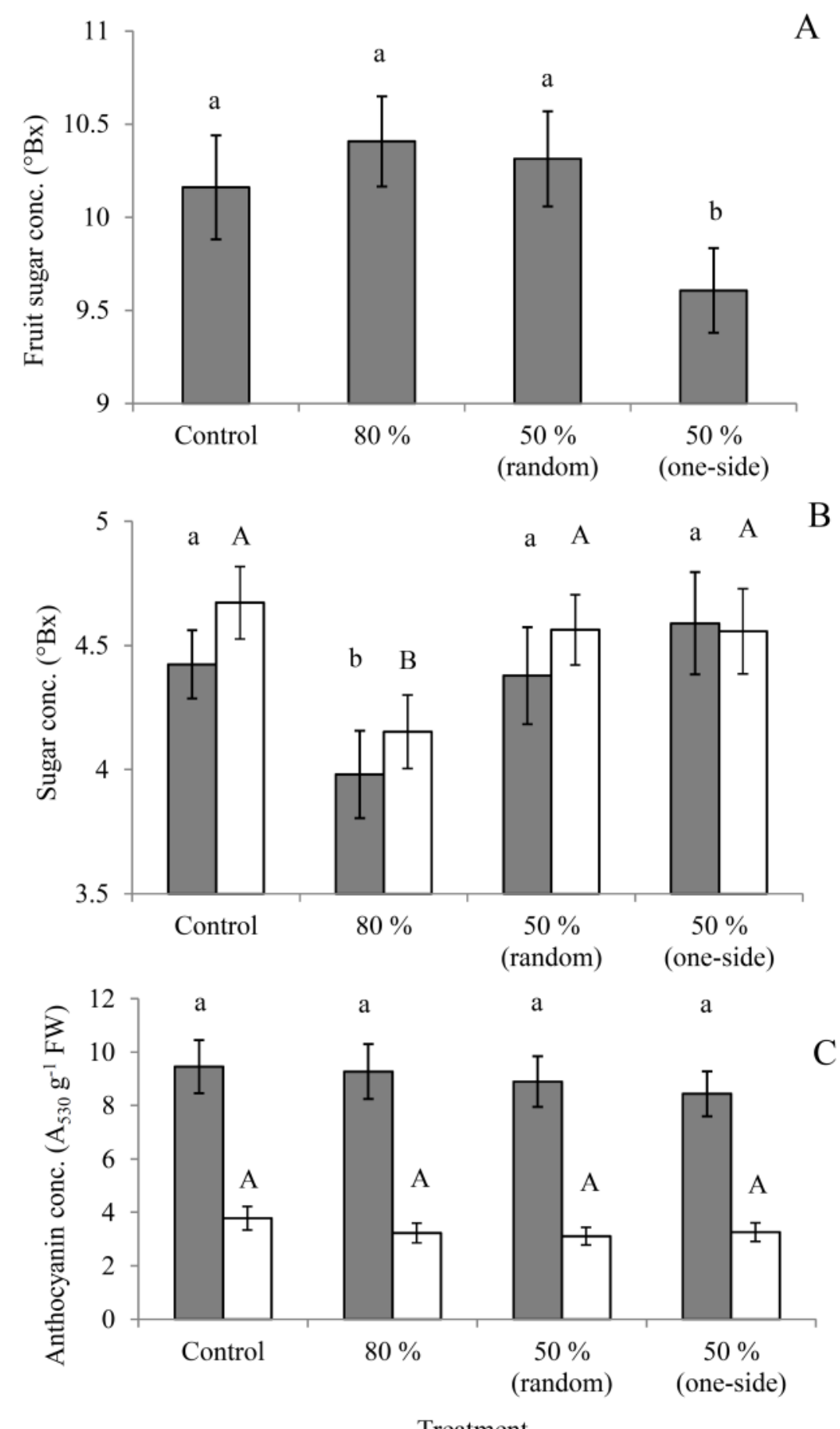

Treatment

Fig 3.5. Mean ( \pm SE) soluble sugar for fruit $(A)$, peduncle (B, closed bars) and ray (B, open bars), as well as anthocyanin concentrations in the peduncle $(\mathrm{C}$, closed bars) and ray $(\mathrm{C}$, closed bars) of ripe Sambucus nigra infructescences. Treatments: (Control) all fruits retained; $80 \%$ of fruits removed before ripening; $50 \%$ fruits were removed randomly across infructescence; $50 \%$ of fruits removed from one side of the infructescence. $n=46$. Letters above bars indicate statistically significant differences between treatments $(\mathrm{P}<0.001)$. 


\subsubsection{Shade experiment}

Those peduncles and rays that were shaded by a neutral grey polycarbonate filter while they were green and bearing unripe fruits, remained green after fruit maturation. In contrast, those organs exposed to full sunlight turned red, irrespective of whether they were rays borne on the same infructescence, or rays and peduncles on adjacent infructescences. Anthocyanin concentrations were significantly lower in artificially shaded than unshaded portions of rays borne on the same infructescence $(P<0.01)$, or both peduncles and rays on adjacent infructescence $(P<0.05$ in each instance; Fig 3.6). One shaded ray and one whole infructescence abscised during development, reducing the number of replicates to nine pairs of peduncles, and eight pairs of rays.

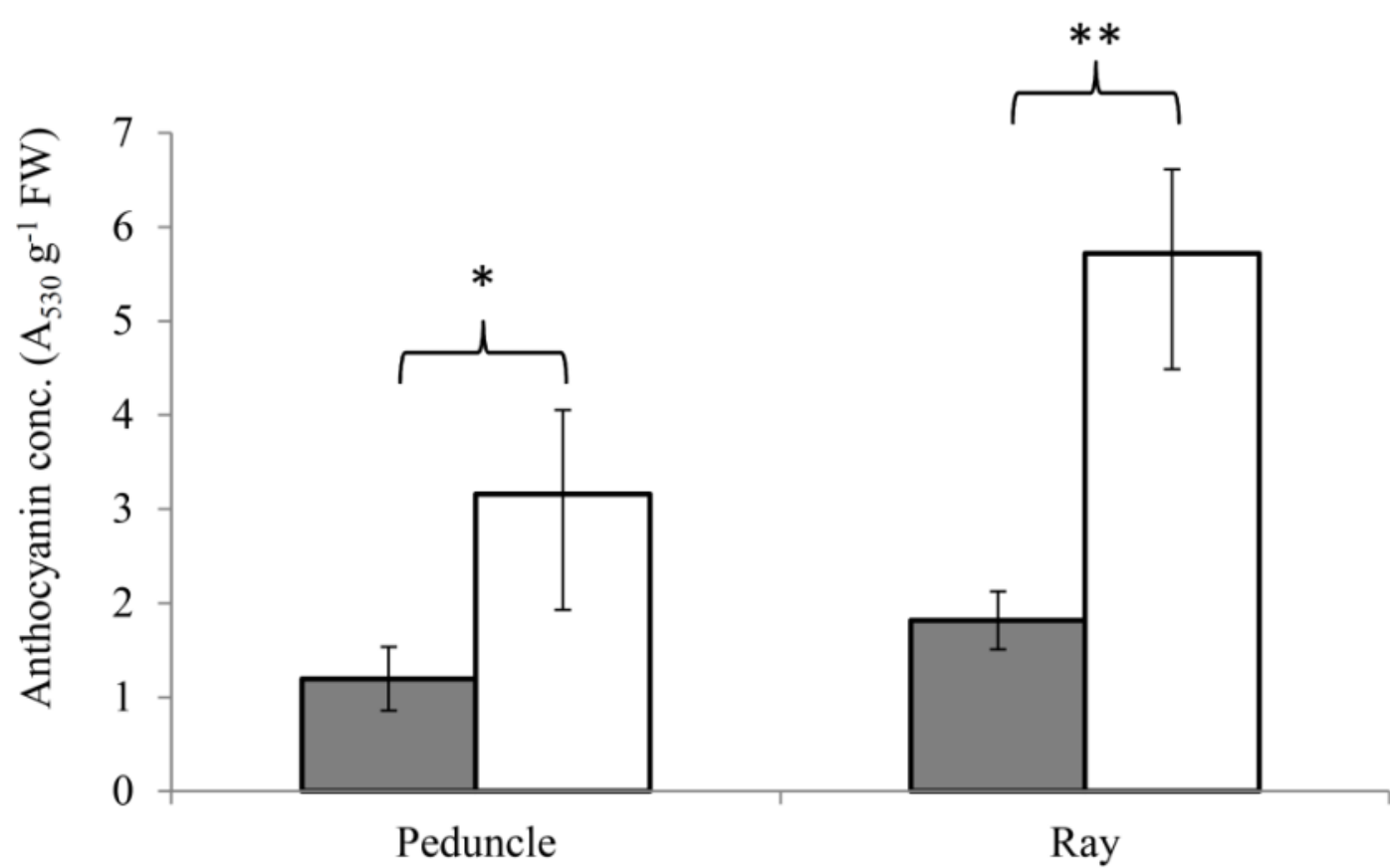

Fig 3.6. Mean ( \pm SE) anthocyanin concentrations for Sambucus nigra peduncles $(n=9)$ and rays $(n=8)$ that were artificially shaded (closed bars) or exposed to full sunlight (open bars) during development. * $P<0.05 ; * * P<0.01$.

\subsubsection{Chlorophyll fluorescence light response curves for S. nigra peduncles}

The $F v / F m$ values of dark-acclimated material were low, but comparable for red $(0.54 \pm$ $0.01)$ and green $(0.6 \pm 0.04)$ peduncles. When subjected to a light ramp ascending to 1700 $\mu \mathrm{mol} \mathrm{m} \mathrm{m}^{-2} \mathrm{~s}^{-1}, \Phi_{P S I I}$ declined more in green than in red peduncles $(P<0.01$; Fig 3.7$)$ such that under the highest irradiance, which approached that of full midday sunlight, $\Phi_{P S I I}$ values in 
green peduncles were only $52 \%$ of those in red ones. $q P$ was consistently greater in red peduncles $(P<0.05)$; NPQ was $67 \%$ greater in green peduncles with $50 \mu \mathrm{mol}$ photons $\mathrm{m}^{-2} \mathrm{~s}^{-1}$ $(P=0.02)$, but was comparable across the two colours when illuminated with between 250 and $1700 \mu \mathrm{mol}$ photons $\mathrm{m}^{-2} \mathrm{~s}^{-1}(P>0.05 ;$ Fig 3.7). 

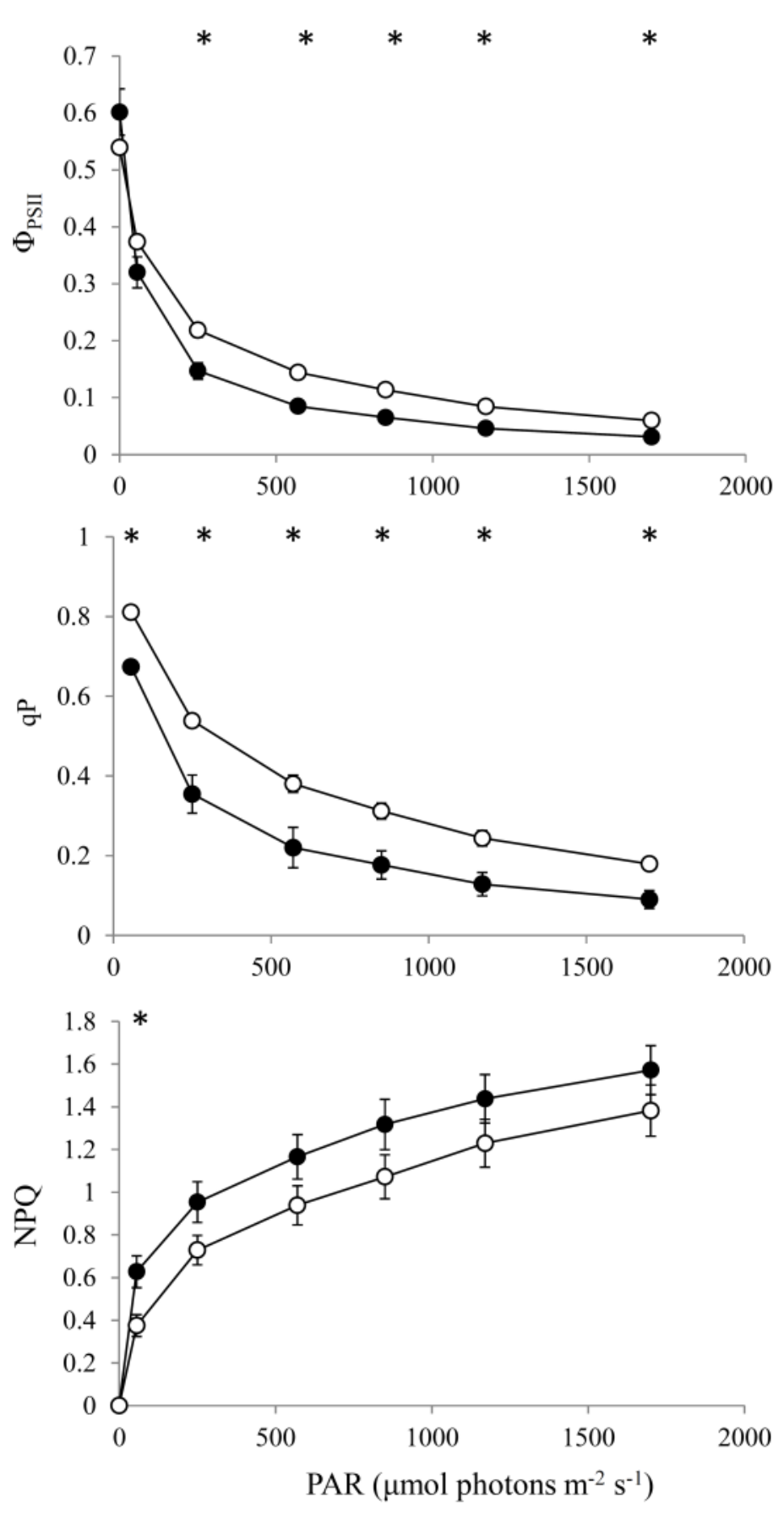

Fig 3.7. Rapid light response curves for photochemical quantum yield (ФPSII), photochemical quenching (qP) and non-photochemical quenching (NPQ) for red ( $\mathrm{O}$ ) and green $(\bullet)$ peduncles. Means $\pm \mathrm{SE}, \mathrm{n}=6, *$ indicates statistically significant differences between groups. 


\subsubsection{Photoinhibitory responses of peduncles}

For those peduncles subjected to photoinhibitory light treatments, dark-acclimated $F v / F m$ was comparable for the base and apex of both the entirely-green $(0.63 \pm 0.02$ and $0.67 \pm 0.2$ respectively) and the variegated $\mathrm{red} / \mathrm{green}(0.60 \pm 0.03$ and $0.61 \pm 0.2$ respectively $)$ peduncles. When subjected to $1200 \mu \mathrm{mol}$ photons $\mathrm{m}^{-2} \mathrm{~s}^{-1}$ white light for $4 \mathrm{~h}$ and then $1 \mathrm{~h}$ reacclimation in the dark, the decline in $F v / F m$ was almost 30 times greater for the green base, compared to the red apex, of variegated peduncles $(P<0.01$; Fig 3.8A). In contrast, the decline in $F v / F m$ was comparable $(P=0.8)$ between basal and apical regions of the all-green peduncles.

For those peduncles subjected to $500 \mu \mathrm{mol}$ photons $\mathrm{m}^{-2} \mathrm{~s}^{-1}$ monochromatic blue light, no difference in the decline of quantum yield $(\Delta F v / F m)$ was found between red and green peduncles $(n=6 ; P=0.11)$. In contrast, the decline in quantum yield was $250 \%$ higher for green peduncles, compared to red, when subjected to green light $(n=6 ; P<0.001)$ and $47 \%$ higher for green peduncles, compared to red, under red light $(n=6 ; P=0.03$, Fig 3.8B).
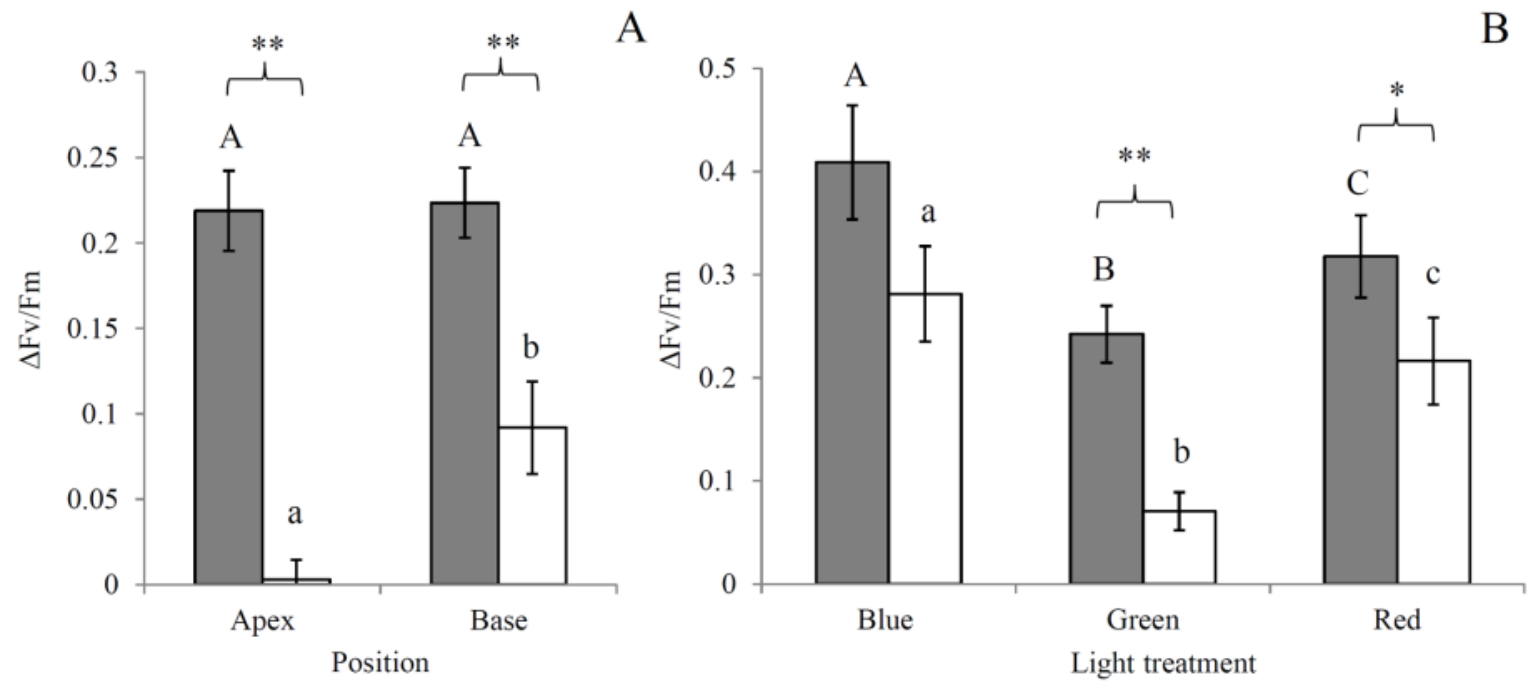

Fig 3.8. Decline in quantum yield after (A) $4 \mathrm{~h}$ exposure to $1200 \mu \mathrm{mol}$ photons $\mathrm{m}^{-2} \mathrm{~s}^{-1}$ white light and $1 \mathrm{~h}$ re-acclimation in the dark, for entirely green (closed bars) and mixed red/ green (open bars), or (B) $6 \mathrm{~h}$ exposure to $500 \mu \mathrm{mol}$ photons $\mathrm{m}^{-2} \mathrm{~s}^{-1}$ blue, green or red light for entirely green (closed) and entirely red (open), Sambucus nigra peduncles. Means \pm SE, $n=$ 10. Letters above bars indicate statistically significant differences between (above) top and bottom of peduncle, or (below) light treatment $(P<0.01)$. Statistically significant difference between colour types: $* *, P<0.01$. 


\subsubsection{Light response curves and photoinhibitory responses for petioles}

Levels of dark-acclimated $F v / F m$ were comparable for red $(0.76 \pm 0.01)$ and green $(0.74 \pm$ $0.01)$ petioles. When subjected to an irradiance ramp peaking at $1700 \mu \mathrm{mol}$ photons $\mathrm{m}^{-2} \mathrm{~s}^{-1}$, levels of quantum efficiency ( $\left.\Phi_{\mathrm{PSII}}\right)$ were comparable up to $900 \mu \mathrm{mol}$ photons $\mathrm{m}^{-2} \mathrm{~s}^{-1}$ for red and green petioles, but at irradiance levels of $1200 \mu \mathrm{mol}$ photons $\mathrm{m}^{-2} \mathrm{~s}^{-1}$ and, approaching the strength of full sunlight, $1700 \mu \mathrm{mol}$ photons $\mathrm{m}^{-2} \mathrm{~s}^{-1}$, levels of quantum efficiency were $52 \%$ higher in red petioles compared to green $(P<0.05$; Fig 3.9). Similarly, levels of $q P$ were comparable when irradiated at light intensities between $6 \mu \mathrm{mol}$ photons $\mathrm{m}^{-2} \mathrm{~s}^{-1}$ and $500 \mu \mathrm{mol}$ photons $\mathrm{m}^{-2} \mathrm{~s}^{-1}$, but were up to $35 \%$ higher in red petioles at intensities between $900 \mu \mathrm{mol}$ photons $\mathrm{m}^{-2} \mathrm{~s}^{-1}$ and $1700 \mu \mathrm{mol}$ photons $\mathrm{m}^{-2} \mathrm{~s}^{-1}(P<0.01)$. NPQ was comparable for red and green petioles at all light intensities (Fig 3.9).

For those leaves subjected to $1200 \mu \mathrm{mol}$ photons $\mathrm{m}^{-2} \mathrm{~s}^{-1}$ white light for $4 \mathrm{~h}$, the depression in $\mathrm{Fv} / \mathrm{Fm}$ was $57 \%$ higher in green petioles compared to red, when irradiated at $21{ }^{\circ} \mathrm{C}\left(t_{5}=4.7\right.$; $P=0.005)$, and $93 \%$ higher when irradiated at $4{ }^{\circ} \mathrm{C}\left(t_{5}=8.6 ; P<0.001\right.$; Fig 3.10$)$. 

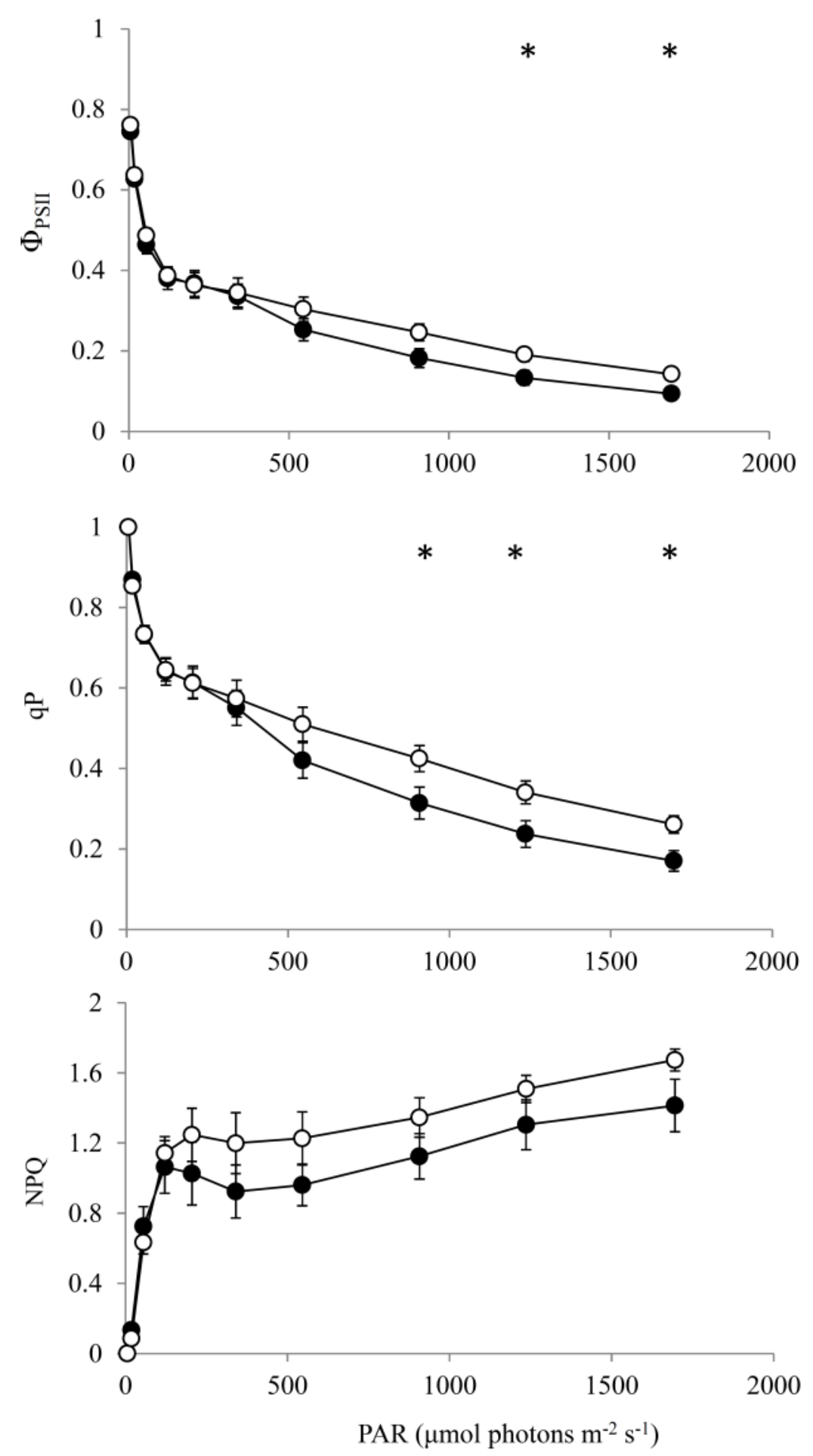

Fig 3.9. Light response curves for photochemical quantum yield (Fs/Fm'), non-photochemical quenching (NPQ) and photochemical quenching of photosystem II for green and red Sambucus nigra petioles. Means $\pm \mathrm{SE}, n=6$. Statistically significant difference between colour types: * $P<0.05$. 


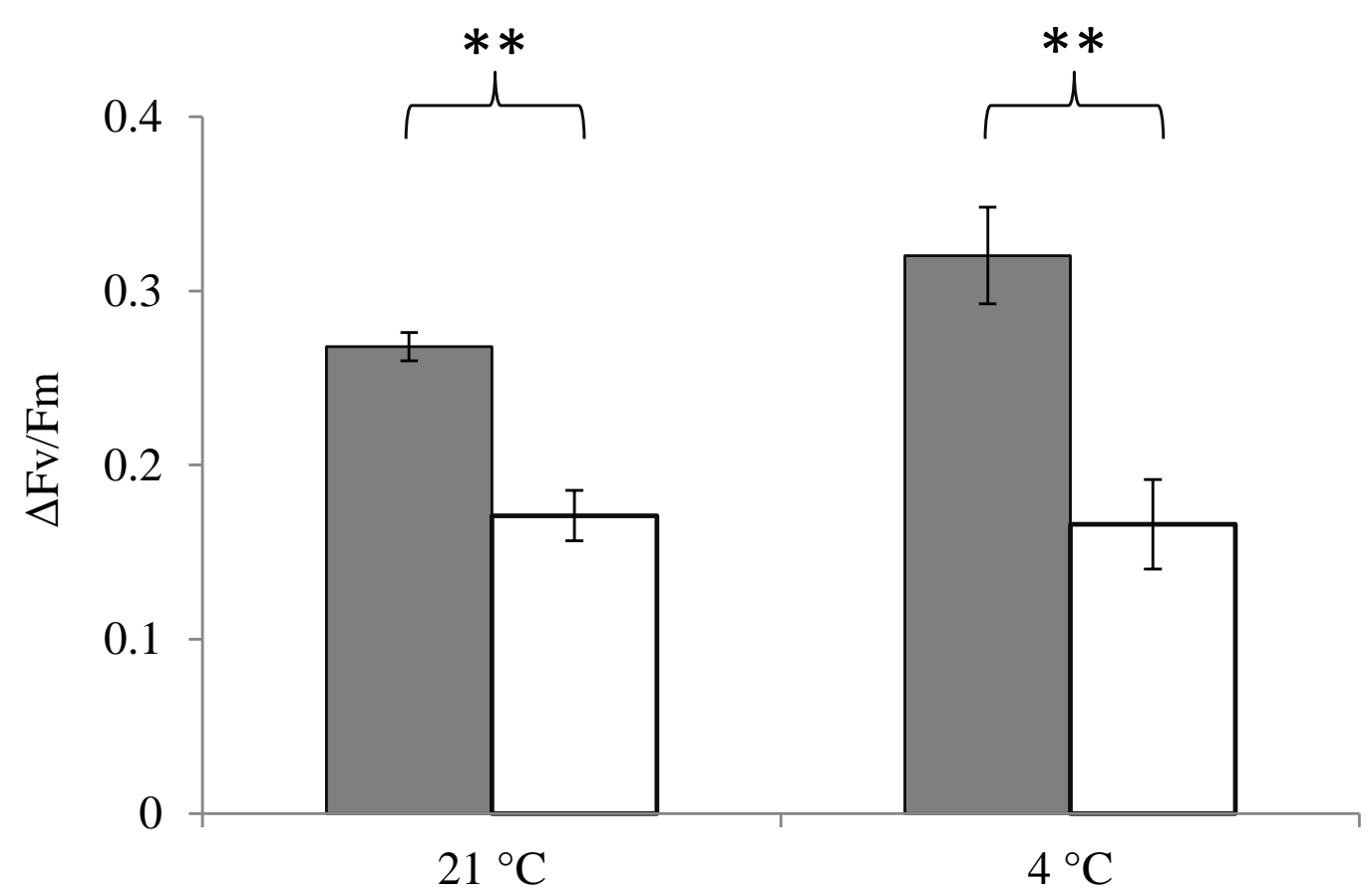

Fig 3.10. Depression in $F v / F m$ for red and green petioles after exposure to four hours of $1200 \mu \mathrm{mol}$ photons $\mathrm{m}^{-2} \mathrm{~s}^{-1}$ white light at $21^{\circ} \mathrm{C}$, and $4^{\circ} \mathrm{C}$. Means $\pm \mathrm{SE}, n=6$. Statistically significant difference between colour types: $* *, P<0.01$.

\subsection{Discussion}

Four sets of data from this analysis support a photoprotective function for anthocyanin accumulation in S. nigra peduncles. First, anthocyanin production increases in response to exposure to full sunlight (Fig 3.6). Second, anthocyanins significantly reduced the amount of photosynthetically active radiation (PAR) transmitted to subjacent chlorenchyma tissue (Fig 3.2). Third, quantum efficiency $\left(\Phi_{P S I}\right)$ was significantly higher for red than for green peduncles during exposure to high light levels (Fig 3.7). Finally, the green base of variegated $\mathrm{red} /$ green peduncles incurred a larger decline in $\mathrm{Fv} / \mathrm{Fm}$ than did the red apex following light stress, while the apex and base of peduncles that were entirely green incurred comparable reductions in $F v / F m$ (Fig 3.8). In contrast, little support was found for a role in frugivore attraction for peduncle reddening. Anthocyanin concentrations in the peduncle were a reliable indicator of fruit quality, but manipulating reward quality, either by a reduction in crop size, or a reduction in both, crop size and fruit quality, had no effect on peduncle colour (Fig 3.5). Moreover, no relationship was found between peduncle colour and frugivory (see section 
3.4.2). Collectively, these data present a compelling case for a light-screening role for anthocyanins in S. nigra peduncles, and argues against this colour change acting primarily as an adaptation to promote frugivore attraction.

\subsubsection{Support for a physiological function for anthocyanins in S. nigra peduncles}

Plants employ several strategies to deal with excess quanta (Takahashi \& Badger, 2011); isolating the specific contribution of anthocyanins to photoprotection can, therefore, be challenging (Steyn et al., 2002). For S. nigra, I found that the green peduncles displayed a lower $\Phi_{P S I I}$ and $q P$ under saturating light (Fig 3.7), as well as greater reductions in $F v / F m$ following light stress (Fig 3.8). Given that red peduncles were sun-acclimated and green peduncles were shade-acclimated, it is therefore not surprising that red peduncles performed better under high light. However, for those sun-acclimated peduncles that were variegated and for which the decline in $F v / F m$ was greater in the green basal portions than in the red apical portions, the difference cannot be attributed to light-acclimation. Nor can the differences between base and tip easily be explained by positional or age effects, since those peduncles that were entirely green showed no difference in $\Delta F v / F m$ between these regions. The sub-epidermal anthocyanins, which were present only in red portions of the peduncle (Fig 3.1) provide the most likely explanation for these observations. Accordingly, when peduncles were exposed to monochromatic green light (i.e., the waveband absorbed maximally by anthocyanins), $\Delta F v / F m$ was greater for the green than the red peduncles, but no difference was found between the two under monochromatic blue light (ie. the waveband anthocyanins do not absorb). Thus, anthocyanins appear to act as light screens, as has been observed in red leaves and stems (Shao et al., 2008; Gould et al., 2010; Zhang et al., 2011; Hatier et al., 2013). However, a light-screening function cannot easily explain why under monochromatic red light the green peduncles showed a larger decline in $F v / F m$ compared to the red ones, since the transmittance of red light was not altered by the presence of anthocyanins (Fig 3.2). The NPQ data (Fig 3.7) indicated that there was no substantial difference in xanthophyll cycle activity between red and green peduncles (Fig 3.7). Thus, it appears that in addition to light screening, there are other photoprotective mechanisms operating in red peduncles. Anthocyanins have been shown to exert antioxidant activity in addition to their role as light filters and for Arabidopsis thaliana, anthocyanic leaves displayed higher quantum efficiencies of PSII under high irradiance compared to acyanic leaves, even when anthocyanin concentrations were too low to be an effective light screen 
(Zhang, et al., 2012). It might be that the differences in $\Delta F v / F m$ identified between red and green peduncles result from anthocyanins acting as direct scavengers of ROS.

A requirement for photoprotection, as provided by anthocyanin accumulation, may be especially important for $S$. nigra peduncles during senescence. For autumnal leaves, anthocyanic reddening coincides with an extended period of senescence (Schaberg et al., 2008; Zhang et al., 2013b), and is associated with improved nitrogen recovery before abscission (Feild et al., 2001; Hoch et al., 2001; Hoch et al., 2003) although exceptions to this association have been noted (Misyura et al., 2012). While the mechanism by which this is achieved is unknown, the photoprotective properties of anthocyanins have been implicated. Ethylene and auxin are among the hormones responsible for coordinating senescence in both leaves and peduncles, with ethylene accelerating and auxin retarding the process (Lyons \& Widmer, 1984; Goszczynska \& Zieslin, 1993; Roberts et al., 2000; Roberts et al., 2002; Schaberg et al., 2008). Oxidative stress appears to hasten leaf senescence by increasing a tissue's sensitivity to ethylene while simultaneously reducing auxin transport (Michaeli et al., 1999a; Michaeli et al., 1999b). By preventing photoinhibition and subsequent ROS production (Schaberg et al., 2008), anthocyanins may be responsible for prolonging leaf senescence, and this function may similarly be present in S. nigra peduncles. This study demonstrates the light screening potential and photoprotective function of anthocyanins in $S$. nigra peduncles, but has not tested whether anthocyanins similarly prolong senescence. As seen in leaves, prolonging senescence would enable more time for nitrogen reabsorption into the plant. Additionally, and uniquely relevant to peduncles, prolonging senescence would enable fruits to be presented for longer, thereby increasing the chances of fruit dispersal. Future studies should investigate whether anthocyanins prolong senescence and improve nitrogen resorption in peduncles, as has been proposed for anthocyanins in senescing leaves.

\subsubsection{Is peduncle colour a signal?}

That anthocyanin accumulation coincides with peduncle senescence may have important implications for our understanding of the evolution of communicative functions in peduncles. These data (Fig 3.4) and those of Schaefer and Braun (2009), show that to an approaching frugivore, peduncle colour provides a reliable cue of the nutritional reward of $S$. nigra fruits; the redder peduncles bear fruits with higher soluble sugar content. However, because fruit sugar content increases during ripening, and anthocyanin content in the peduncle increases during senescence, both parameters increase over time. Those infructescences collected late 
in their fruiting period would be expected to have the most sugary fruits, and as anthocyanin had accumulated for a longer duration, we would expect these infructescences to bear the reddest peduncles. Similarly, differences in fruit sugar content between infructescences bearing red or green peduncles may simply be because red infructescences tend to ripen earlier in the season. Thus, the information peduncle reddening imparts on fruit quality may be a by-product of temporal variability in fruit ripeness, and may not represent an adaptation to communicate nutritional investment.

In this study, we tested the relationship between peduncle colour and fruit sugar by manipulating sugar content of ripe fruits and quantifying anthocyanin content in adjacent peduncles. If peduncle colour were an adaptation to signal fruit reliability, we would expect that reducing fruit sugar content would similarly reduce peduncle colour. However, a reduction in the sugar content of ripe fruits, achieved by manipulating crop size while fruits were unripe, did not coincide with a reduction in anthocyanin concentrations in the peduncle. Evidently, the correlation between peduncle colour and fruit sugar that Schaefer and Braun identified (2009) breaks down when fruit sugar is manipulated.

In infructescences bearing green peduncles, Schaefer and Braun (2009) identified a negative correlation between a peduncle's chlorophyll content, and sugar content of that peduncle's fruits. Moreover, low chlorophyll concentrations gave peduncles a lighter hue, thereby increasing the chromatic contrast of the fruits they bore. Schaefer and Braun (2009) suggested that a reduced chlorophyll content in the peduncle may be a potential handicap signal to approaching herbivores; high-quality plants capable of producing sugary fruits are similarly capable of sacrificing chlorophyll content in the peduncle. This would in turn enhance fruit conspicuousness while advertising fruit quality. However, this scenario fails to acknowledge that fruit maturation coincides with peduncle senescence, a process by which chlorophyll is catabolised. Once again, concentrations of both, fruit sugar and peduncle chlorophyll, change over time (with sugar increasing while fruits ripen and chlorophyll decreasing while peduncles senesce). The negative correlation that Schaefer and Braun (2009) identified may also have arisen because the infructescences collected were of varying ripeness.

\subsubsection{Peduncle colour and frugivory?}

Schaefer and Braun (2009) have previously demonstrated that peduncle reddening influences fruit consumption for $S$ nigra, both, in a cafeteria-style choice experiment amongst captive 
blackcaps (Sylvia atricapilla), and when red or green infructescences are excised and presented in a natural environment. Although Schaefer and Braun's study provided reliable data on the specific effect of peduncle colour on fruit choice, successful seed dispersal is also influenced by a number of other traits. The duration of the fruiting period, or the environment specific to green peduncles may influence frugivory (Levey, 1990), or various stochastic factors, such as bird migration patterns may simply negate any benefit imparted by peduncle colour. In this study, I measured frugivory for 24 infructescences of varying peduncle colour, for the duration of their respective fruiting periods, and did so on the plants from which the infructescences were borne. Here, no relationship was found between the number of fruits removed and anthocyanin content in the peduncle (see section 3.4.2), a result which suggests that peduncle colour may not significantly influence fruit consumption. While it may be that additional replicates are needed to identify a difference in frugivory, it may also be that the difference in fruit removal identified by Schaefer and Braun (2009) gets overridden by other factors which influence frugivore visitation such as bird migration patterns.

\subsubsection{Reddening in S. nigra petioles}

If anthocyanins in S. nigra peduncles are a photoprotective adaptation comparable to that described for senescing leaves, it is natural to ask whether the leaves of S. nigra similarly turn red when they senesce. Like the reddening described for S. nigra peduncles, petioles turned red as they were senescing and reddening was exclusive to those leaves exposed to full sunlight (Fig 3.3). Moreover, reddening in both organs was attributable to an anthocyanic layer superjacent to photosynthetic tissues; in peduncles anthocyanins appeared in the outermost hypodermal collenchyma, while in petioles, anthocyanins appeared across a subepidermal layer. I also identified several results consistent with anthocyanins performing a photoprotective function in S. nigra petioles. Red petioles maintained higher quantum efficiencies compared to green petioles, when both were exposed to high light levels (Fig 3.9), and red petioles exhibited a lower depression in $F v / F m$ following light stress (Fig 3.10). Evidently, the colour change in S. nigra peduncles displays characteristics comparable to that of petioles (i.e. histological distribution, environmental determination, and timing relative to senescence), and imparts a physiological benefit comparable to that of peduncles. For this reason, it might be that anthocyanins in these two organs perform a comparable function. However, petiole anthocyanins accumulate after the fruiting season, excluding the possibility 
that they promote frugivore attraction. If anthocyanins in petioles and peduncles are produced to perform the same adaptive function, that function cannot be frugivore attraction.

\subsubsection{Other considerations and limitations}

A null result was found when comparing peduncle anthocyanin content for those infructescences whose crop size had been manipulated. My study showed that manipulating sugar content, either in the fruit or in the peduncle, had no effect on total anthocyanins in the peduncle or ray (Fig 3.5). While this result suggests that peduncle colour does not correlate with peduncle sugar or fruit sugar content, it might reflect a limitation of the experimental design. It could be argued that peduncle colour did indeed evolve to signal fruit quality, and that the two are intrinsically linked, but that my manipulation of sugar content was not sufficient to alter peduncle colour. For instance, the fruit sugar content of those infructescences from which all fruits were removed from two of an infructescences four rays, was only $5 \%$ less than those control infructescences from which no fruits were removed (Fig 3.5). While this experiment provides a novel means of manipulating both fruit and peduncle sugar content, a link between sugar and anthocyanins cannot be entirely dismissed.

One final limitation of this data set is that I have not shown whether reducing light stress in $S$. nigra peduncles improves an individual's fitness. Without doing so, we cannot know if this function has been selectively favoured, or is simply a by-product of anthocyanins produced for some other reason. It should be noted that this limitation is common, and to date, no study has shown that photoprotection via anthocyanin accumulation infers a fitness advantage (see section 1.3.2). One can only speculate as to why this aspect of anthocyanin research has yet to be addressed, but it may simply be that fitness comparisons are especially difficult. Luckily, peduncle reddening in Sambucus spp. provides us with a unique opportunity to test whether anthocyanic photoprotection improves an individual's fitness. As discussed above, foliar reddening during senescence and the photoprotection it imparts have been associated with a prolonged senescence duration (Schaberg et al., 2008). If anthocyanins in peduncle tissue similarly prolong senescence, fruits borne on those peduncles could be presented for longer. Conversely, peduncles that senesce and abscise too quickly would incur a greater fruit mortality and lower fitness. 


\subsection{Conclusion}

Anthocyanins in S. nigra peduncles appear to protect photosynthetic tissue from the harmful effects of excess light. However, I found little evidence to support a communicative function previously attributed to peduncle reddening. Few studies simultaneously explore the physiological benefits anthocyanins impart, as well as the role of anthocyanic colour in plantanimal communication, and these data highlight the importance of correcting this. 


\section{Chapter 4: Do anthocyanins prolong senescence in Sambucus canadensis peduncles?}

\subsection{Abstract}

It has been suggested that anthocyanin accumulation prolongs senescence in leaves, yet, for other organs, this benefit has not been tested. Here, I hypothesised that anthocyanin accumulation in senescing Sambucus canadensis peduncles mitigates light stress, prolongs senescence and enhances nitrogen resorption. Red peduncles possessed several traits indicative of a prolonged senescence; their rates of chlorophyll and xanthophyll decline were lower (22\% and 34\% respectively), while tensile strength and elasticity were higher (120\% and $78 \%$ respectively) than for green peduncles. Red peduncles were also less susceptible to photoinactivation than the green ones at the later stages of senescence. However, manipulating green peduncles with light filters possessing transmittance properties comparable to an anthocyanic tissue layer did not increase peduncle longevity or nitrogen resorption, nor did it reduce ROS, a product of photoinhibition presumed to accelerate senescence. I conclude that like senescing leaves, red peduncles display many characteristics indicative of a prolonged senescence, but I am unable to attribute this benefit to the presence of anthocyanins.

\subsection{Introduction}

Ameliorating light stress, prolonging senescence and enhancing nitrogen resorption are among the putative functions proposed for anthocyanins in senescing leaves (Feild et al., 2001; Hoch et al., 2001; Lee, 2002; Hoch et al., 2003; Schaberg et al., 2003; Schaberg et al., 2008; Archetti et al., 2009; Manetas \& Buschmann, 2011; Zhang et al., 2013b) and a requirement for these functions may similarly explain the accumulation of anthocyanins in other senescing organs. Peduncles of Sambucus sp. accumulate anthocyanins when infructescences ripen, a response that coincides with senescence and demonstrably mitigates the severity of light stress (see Chapter 3). However, it is yet to be shown whether abating light stress might prolong senescence or enhance nitrogen resorption from peduncles back into a plant's branches, benefits comparable to those proposed for autumnal leaves. Thus, it is 
yet to be shown whether avoiding light stress in peduncles is a characteristic of importance to an individual's fitness. Moreover, prolonging senescence would impart an additional benefit in peduncles: extending the period of fruit presentation.

Senescence represents the final stage of an organ's ontogeny. During this period, proteins, membranes, lipids and chloroplasts, are catabolised (Guo \& Gan, 2005; Lim et al., 2007), the products of which are then exported to other organs in the plant. Termed nutrient recycling, this process is present in roots, shoots and leaves (Freschet et al., 2010), and is seen as a significant contributor to plant fitness (May \& Killingbeck, 1992). Chloroplasts can contain up to $70 \%$ of a photosynthetic organ's nitrogen content (Lim et al., 2007), and thus, are especially significant contributors to nutrient recycling. However, successful recycling of nitrogen may be impaired by light stress, (Feild et al., 2001), and photosynthetic tissues are particularly susceptible to light stress during this period. Ameliorating the severity of light stress may therefore be critical to optimising nitrogen resorption during senescence.

Light stress encompasses a number of conditions, all of which are derived from receiving light energy in excess of that needed for photosynthesis. Initially characterised by a decline in photosynthetic yield, prolonged exposure can lead to photoinactivation, when photodamage exceeds the rate at which PSII can be repaired, and subsequently, photoinhibition (Murata et $a l .$, 2007), the long-term damage of the PSII repair mechanisms (Takahashi \& Murata, 2008; Tyystjärvi, 2008). Photoinhibition can also coincide with the production of reactive oxygen species (ROS), a family of molecules, which in excess, cause damage to proteins, lipids and DNA (Bartosz, 1997). By absorbing light energy that would otherwise be intercepted by photosynthetic tissues, anthocyanins are thought to ameliorate the effects of light stress. Numerous studies have reported a reduction in the severity of photoinhibition for red leaves and stems, compared to green, when exposed to excess light (Gould et al., 1995; Dodd et al., 1998; Feild et al., 2001; Manetas et al., 2002; Steyn et al., 2002; Gould, 2004; Hughes et al., 2005; Hatier \& Gould, 2009; Gould et al., 2010; Hughes et al., 2014), although exceptions have been noted (Esteban et al., 2008; Zeliou et al., 2009).

Anthocyanin accumulation has evolved multiple times in senescing leaves (Archetti, 2009c), which suggests that anthocyanins impart a selective advantage. By preventing photoinhibition, and subsequent ROS production, anthocyanins are thought to enhance nutrient recycling in senescing tissues (Feild et al., 2001; Hoch et al., 2001). While some studies found no support for this hypothesis (Manetas \& Buschmann, 2011; Misyura et al., 
2012), Field (2001) showed that Cornus stolonifera leaves that turn yellow during senescence exhibit significantly greater levels of photoinhibition than do leaves which senesce red. Moreover, Hoch et al. (2003) showed that when senescing under light and cold stress, acyanic mutants of three deciduous species showed a reduced nitrogen resorption compared to anthocyanic wild type varieties. In the absence of light stress, green varieties display rates of nitrogen resorption comparable to that of red varieties (Feild et al., 2001; Hoch et al., 2003). Nutrient recycling may also be enhanced by prolonging senescence, a benefit which anthocyanin accumulation may impart (Schaberg et al., 2008). While the mechanism by which this is achieved is unknown, red senescing species demonstrate a late leaf abscission compared to co-occurring acyanic species (Zhang et al., 2013b) and possess several characteristics indicative of a prolonged senescence (Schaberg et al., 2008). Thus, there are two ways by which anthocyanins are thought to improve nitrogen absorption during senescence: for a given period of time, anthocyanin accumulation may improve the rate at which nutrient resorption may take place, or anthocyanin accumulation may extend the period of nutrient resorption. Whether these benefits, which have received considerable attention in senescing leaves, are similarly applicable to other senescing organs has yet to be tested.

For various species within the genus Sambuccus, fruit development coincides with anthocyanin accumulation in the peduncle and pedicels. While originally considered an adaptation to promote frugivore visitation (Schaefer \& Braun, 2009), in chapter 3 I noted several characteristics in S. nigra which suggest that this trait may instead be a physiological adaptation. First, anthocyanin accumulation precedes fruit maturation, and is only present in those individuals in high light environments. If this was a signalling adaptation, exposed peduncles would be unnecessarily red while fruits are still unripe, and shaded peduncles would remain unnecessarily green. Because reddening precedes abscission, we know that anthocyanin accumulation occurs during senescence. Moreover, I demonstrated that anthocyanins are responsible for ameliorating light stress in peduncles of at least one Sambucus sp. (Chapter 3). Because reddening in Sambucus peduncles shares many characteristics with senescing leaves, we have an ideal opportunity to test whether anthocyanins similarly prolong senescence and improve nitrogen resorption in these tissues.

Using S. canadensis peduncles, I tested the requirements of the hypothesis 'that anthocyanin accumulation prevents light stress, prolongs senescence and enhances nitrogen resorption'. Firstly, I compared, for red and green peduncles, the rate at which chlorophyll and 
xanthophyll concentrations, and photosynthetic health, as estimated by chlorophyll fluorescence, decline during senescence. Secondly, I compared the tensile strength of red and green peduncles, an indicator of abscission layer progression in senescing tissues (dela Fuente \& Leopold, 1968; Schaberg et al., 2008). Lastly, I used a novel experimental design to compare longevity, lipid peroxidation and nitrogen resorption between green peduncles bearing a transparent light filter, and green peduncles bearing a fake anthocyanic light filter. This uniquely produced two treatments with identical developmental histories that differed only by the light profiles transmitted to peduncle tissues.

\subsection{Materials and Methods}

\subsubsection{Study site and plant materials}

Plant material was collected from a natural population on a private rural property in Brunswick, Maine (43.55 N, $70.40 \mathrm{~W}$ ) in the summer/fall of 2013. There, S. canadensis forms a small tree up to $3 \mathrm{~m}$ tall, and is prominent in both the forest interior and open clearings. It flowers from July until August and fruits from mid-August until late October. At this site, S. canadensis was the dominant species on a roadside clearing, bordered by tall pines, and the light environment ranged from individuals exposed to full sunlight to those shaded throughout the day.

For the experiment comparing the longevity of red and green peduncles, five whole plants were collected from a deciduous forest located on another private property $(43.52 \mathrm{~N}, 69.57$ W) and transported to Bowdoin College, Maine (43.54 N, 69.57 W). At the site of collection, S. canadensis naturally occurs in the forest understory, as well as the forest margins. All five individuals collected were from the forest understory, potted while fruits were still unripe, and left to acclimate for five weeks while being watered every two days.

\subsubsection{Chlorophyll florescence parameters before, during, and after fruit maturation}

Twenty infructescences, 10 shaded and 10 exposed, were collected, each from a single plant, while fruits were unripe and peduncles were green. Plants were chosen at random, but infructescences were preferentially selected on the basis that they were either exposed and south facing, and thus receiving sunlight throughout the day, or because they were borne on lower branches, and thus shaded throughout the day. Peduncles were dark-acclimated for $1 \mathrm{~h}$ and the ratio of variable to maximum fluorescence $(F v / F m)$ was measured using a Hansatech 
FMS-2 (Hansatech Instruments Ltd, Norfolk, UK) chlorophyll fluorometer. Each peduncle was then subjected to an irradiance ramp of four steps, each $10 \mathrm{~min}$ long, and peaking at 1600 $\mu \mathrm{mol}$ photons $\mathrm{m}^{-2} \mathrm{~s}^{-1}$ of white light issued by the fluorometer, which was then maintained for $2 \mathrm{~h}$. Every $10 \mathrm{~min}$, photochemical quantum yield $\left(\Phi_{P S I I}\right)$ and non-photochemical quenching $(N P Q)$ were measured. Following the light treatment, peduncles were returned to the dark and the difference in the ratio of variable to maximum fluorescence $(\Delta F v / F m)$ between peduncles pre-treatment and peduncles post-treatment, was then measured at 10 min intervals for an additional $40 \mathrm{~min}$ This process was then repeated for an additional 14 infructescences when fruits began to ripen and exposed peduncles turned red, and again for an additional 20 plants after fruits had been naturally removed. Once again, plants were randomly selected and one infructescence was selected per plant. From half of the plants, exposed infructescences were preferentially selected and for the remaining half, shaded infructescences were preferentially selected. For those infructescences collected while fruits were ripening, infructescences were also preferentially selected such that all were at a comparable stage of ripeness i.e the proportion of ripe to unripe fruits, as determined by fruit colour, was approximately 50/50 for each infructescence. Following each light treatment, peduncles were frozen at $-80{ }^{\circ} \mathrm{C}$ for subsequent analysis of chlorophyll, carotenoid and anthocyanin concentrations.

\subsubsection{Anthocyanin, chlorophyll and carotenoid quantification}

For those infructescences collected in section 4.3.2, two central portions were removed from each peduncle, each portion approximately $5 \mathrm{~mm}$ long, and from one, anthocyanins were extracted in $\mathrm{MeOH}: \mathrm{H}_{2} \mathrm{O}: \mathrm{HCl}$ (16:3:1 v:v:v), and from the other, chlorophylls and carotenoids were extracted in Acetone: $\mathrm{H}_{2} \mathrm{O}$ (8:2). Samples were weighed, ground by mortar and pestle, clarified by centrifugation at $16,000 \mathrm{~g}$ and absorbance peaks measured using a Beckman DU 640 UV/Vis (Brea, CA, USA) spectrophotometer. Anthocyanins concentrations were estimated as $\mathrm{A}_{530} \mathrm{~g}^{-1} \mathrm{FW}$, while chlorophyll and carotenoid concentrations were determined from $\mathrm{A}_{663}, \mathrm{~A}_{647}, \mathrm{~A}_{470}$ using the formulae described by Lichtenthaler (1987).

\subsubsection{Comparative rates of xanthophyll de-epoxidation}

From 20 plants, 10 bearing green peduncles and 10 bearing red peduncles, a single infructescence was removed after fruit maturation and dark-acclimated for $1 \mathrm{~h}$. Peduncles were then subjected to $1600 \mu \mathrm{mol}$ photons $\mathrm{m}^{-2} \mathrm{~s}^{-1}$ of white light for 10 mins with non- 
photochemical quenching $(N P Q)$ measured every minute using a Hansatech FMS-2 (Hansatech Instruments Ltd, Norfolk, UK) chlorophyll fluorometer. Immediately after the light treatment, peduncles were snap-frozen in liquid nitrogen and then stored at $-80^{\circ} \mathrm{C}$. Xanthophyll concentrations were then measured by Nneka Nnatubeugo from the biology department at Bowdoin College using high-performance liquid chromatography (HPLC) via a procedure modified after Gilmore and Yamamoto (1991). Samples were analysed with an Agilent 1100 Series liquid chromatograph equipped with an Agilent 1100 diode array detector (Agilent Technologies, Palo Alto, CA, USA) and a YMC Carotenoid $\mathrm{C}_{30}$ reverse phase column ( $5 \mu \mathrm{m}$ particle size, $250 \mathrm{~mm}$ x $4.6 \mathrm{~mm}$ I.D.) (Waters Corporation, Milford, Mass, USA) equipped with a Phenomenex $\mathrm{C}_{18}$ guard column (Phenomenex, Torrance, CA, USA). Absorbance peaks were measured at $445 \mathrm{~nm}$.

\subsubsection{Shading experiment}

From eight randomly selected plants, three infructescences were preferentially selected such that each was of a similar size and exposure. Infructescences were selected when fruits were still unripe and peduncles were green. For one infructescence from each plant, the peduncle was shaded with a Rosco Supergel (Stamford, CT, USA) neutral density polycarbonate filter (\#398), which permitted the transmittance of $16 \%$ sunlight but did not alter the spectral quality of light. For the remaining two infructescences from each plant, one was left exposed while on the other, a transparent polycarbonate light filter (\#00), permitting 100\% transmittance, was applied to the peduncle. Following fruit maturation and fruit removal, the infructescences were removed and anthocyanin concentrations quantified.

\subsubsection{Longevity and nitrogen resorption experiment}

To determine whether the presence of anthocyanins prolongs peduncle senescence, five plants grown in a shaded forest understory, thereby preventing peduncle reddening, were moved into an exposed location. Among the five plants, 22 pairs of infructescences were identified, each pair containing infructescences of comparable size and developmental age, and borne on the same plant. Within each pair, the peduncle of one infructescence was

shaded by a red Rosco Roscolux (Stamford, CT, USA) polycarbonate filer (\#3313), which allowed a spectral transmittance comparable to that of a anthocyanic sub-epidermal layer, as measured in red Sambucus nigra peduncles (Chapter 3). On the remaining infructescence from each pair, a transparent polycarbonate light filer (\#00) was applied to the peduncle. 
Infructescences were inspected daily, and longevity was calculated as the number of days between when treatments began, September $10^{\text {th }}$, and the day each died. Once abscised, samples were stored at $-80{ }^{\circ} \mathrm{C}$, dried at $60{ }^{\circ} \mathrm{C}$ for four days, and sent to the Boston University Stable Isotope Laboratory (Boston, MA, USA) for quantification of \% nitrogen by mass spectrometry (see http://www.bu.edu/sil/quality.htm; accessed August 2014).

For 10 pairs, the predawn ratio of variable to maximum chlorophyll fluorescence $(\mathrm{Fv} / \mathrm{Fm})$ was measured using a Hansatech FMS-2 (Hansatech Instruments Ltd, Norfolk, UK) chlorophyll fluorometer. Measurements were taken at the central region from the exposed side of each peduncle and light filters were temporarily removed for each measurement. Measurements were taken $48 \mathrm{~h}$ after the plant's initial relocation and every $48 \mathrm{~h}$ subsequently until the last infructescence senesced on November $7^{\text {th }}, 2013$. Measurements were postponed $24 \mathrm{~h}$ if rain prevented use of the chlorophyll fluorometer.

For another 7 pairs, totalling 14 infructescences, two rays from each infructescence were selected. One ray per infructescence was shaded using a red Rosco Roscolux (Stamford, CT, USA) polycarbonate filer (\#3313), while on the other, a transparent polycarbonate light filer (\#00) was applied. After $1 \mathrm{wk}$, rays were excised while exposed to full midday sunlight, immediately frozen in liquid nitrogen, and then stored at $-80{ }^{\circ} \mathrm{C}$. Lipid peroxidation was then measured by Michael Walsh from the Biology department at Bowdoin University following a procedure modified after Logan et al. (1998). All samples were ground in a pre-chilled mortar in $750 \mu 15 \%(w / v)$ meta-phosphoric acid, and subsequently mixed with an equal volume of $0.7 \%(\mathrm{w} / \mathrm{v})$ thiobarbituric acid in $30 \%(\mathrm{w} / \mathrm{v})$ trichloroacetic acid. Samples were then incubated in a $95^{\circ} \mathrm{C}$ water bath for $30 \mathrm{~min}$, cooled rapidly and centrifuged at $14000 \mathrm{~g}$ for 10 minutes at $4{ }^{\circ} \mathrm{C}$. Absorbance peaks of the supernatant were then measured at 532 and 600 $\mathrm{nm}$. The absorbance due to thiobarbituric acid-reactive substances (TBARS) was calculated as $\mathrm{A}_{532}-\mathrm{A}_{600}$, and the content of TBARS calculated using a molar extinction coefficient of $1.55 \times 10^{5} \mathrm{M}^{-1} \mathrm{~cm}^{-1}$ (Logan et al, 1998). The content of TBARS provides a measure of lipid peroxidation, and therefore an indirect measure of ROS production.

\subsubsection{Comparative analysis of tensile strength}

The force required to detach an infructescence from its adjacent stem was used an indicator of abscission layer progression. After fruit maturation, a single infructescence, and its adjacent internode, was removed from each of 40 plants, 20 of which bore green peduncles, 
and 20 of which bore red peduncles. The elasticity of each peduncle, and the force required to detach each peduncle from the adjacent stem was measured using an Instron 4301 electromechanical materials testing machine (Norwood, MA, USA). Materials were mounted in the machine, such that the site of peduncle attachment was equidistant to the two grips. Each grip consisted of a small vice, the jaw of which was textured to prevent material slipping, and tightened via screw and nut. The distance between grips was initially set to 100 $\mathrm{mm}$, and extended at $10 \mathrm{~mm} \mathrm{~min}^{-1}$. Force was measured using a strain-gauge-based force beam (error, less than $\pm 0.02 \mathrm{~N}$ ), and extension was measured using a linear variable differential transformer (error, less than $\pm 0.004 \mathrm{~mm}$ ). Data were digitised (12-bit) at $100 \mathrm{~Hz}$. Following breakage, the base of each peduncle was photographed using a Canon PowerShot A450 (Tokyo, Japan) digital camera, and from these images, the surface area of each peduncle's base was determined. Breaking force was calculated as force/cross sectional area $\left(\mathrm{N} / \mathrm{mm}^{2}\right)$ and breaking extension was calculated as the distance between grips at the time of breakage minus the initial distance of $100 \mathrm{~mm}$.

\subsubsection{Statistical analysis}

All statistical analyses were performed using IBM SPSS 20.0 (Chicago, IL, USA). For those infructescences used in the longevity experiment, whose peduncles were shaded with red or transparent light filters, the relationship between filter colour and either longevity, ROS production or nitrogen content was evaluated using a general linear model (GLM), where filter colour and plant were treated as independent variables, fixed and random respectively, and longevity, ROS and nitrogen were each treated as dependent variables. To compare $\mathrm{Fv} / \mathrm{Fm}$ decline between filter colours, the pattern of decline was first determined by curve estimation, and to represent each peduncle's decline, a sigmoidal curve was fitted. This curve was derived using the formula $y=a /(1+\exp (-(x-b) / c))$ where $a$ describes the value at which $F v / F m$ was initially maintained, $b$, the number of days this initial $F v / F m$ was maintained, and $c$, the rate at which $F v / F m$ declined. The relationship between each of these parameters and filter colour was similarly evaluated using a GLM, where once again, filter colour and plant were treated as independent variables, fixed and random respectively, and $a, b$, and $c$ were each treated as dependent variables.

Differences in pigment concentrations over time, or between red and green peduncles, were compared using a two-way ANOVA, where both time and peduncle colour were treated as fixed independent factors and total chlorophyll, anthocyanin and xanthophyll concentrations 
were each treated as dependent variables. T-tests were used for all post hoc analysis, comparisons of anthocyanin concentrations for shaded and exposed tissues, the ratio of epoxidized to de-epoxidized xanthophylls within, and the tensile strength of, red and green peduncles, as well as differences in photosynthetic parameters, derived from chlorophyll fluorescence, for red and green peduncles.

\subsection{Results}

\subsubsection{Sambucus canadensis pigments}

Peduncles of $S$. canadensis varied from entirely red to entirely green (Fig 4.1A-B). Plants bearing red peduncles, and plants bearing green peduncles, were both found in exposed, sunny locations, often adjacent to each other. In contrast, infructescences borne on plants in the shaded forest interior were almost exclusively green. The anthocyanins were located in the vacuoles of a contiguous band of sub-epidermal cells (Fig 4.1C-D), and reddening extended from the pedicels to the base of the peduncle. Anthocyanin accumulation began prior to fruit ripening and reached its highest concentrations after fruits had been removed (Fig 4.2). While fruits were unripe, green peduncles contained significantly higher concentrations of both chlorophyll and carotenoid pigments (Fig 4.2). However, during fruit maturation when peduncles began to senesce, chlorophyll and carotenoid concentrations decreased more rapidly in green peduncles compared to red. Thus, in the final stages of senescence, red peduncles contained 53\% higher concentrations of chlorophyll and 44\% higher concentrations of carotenoid pigments than did green peduncles (Fig 4.2). 


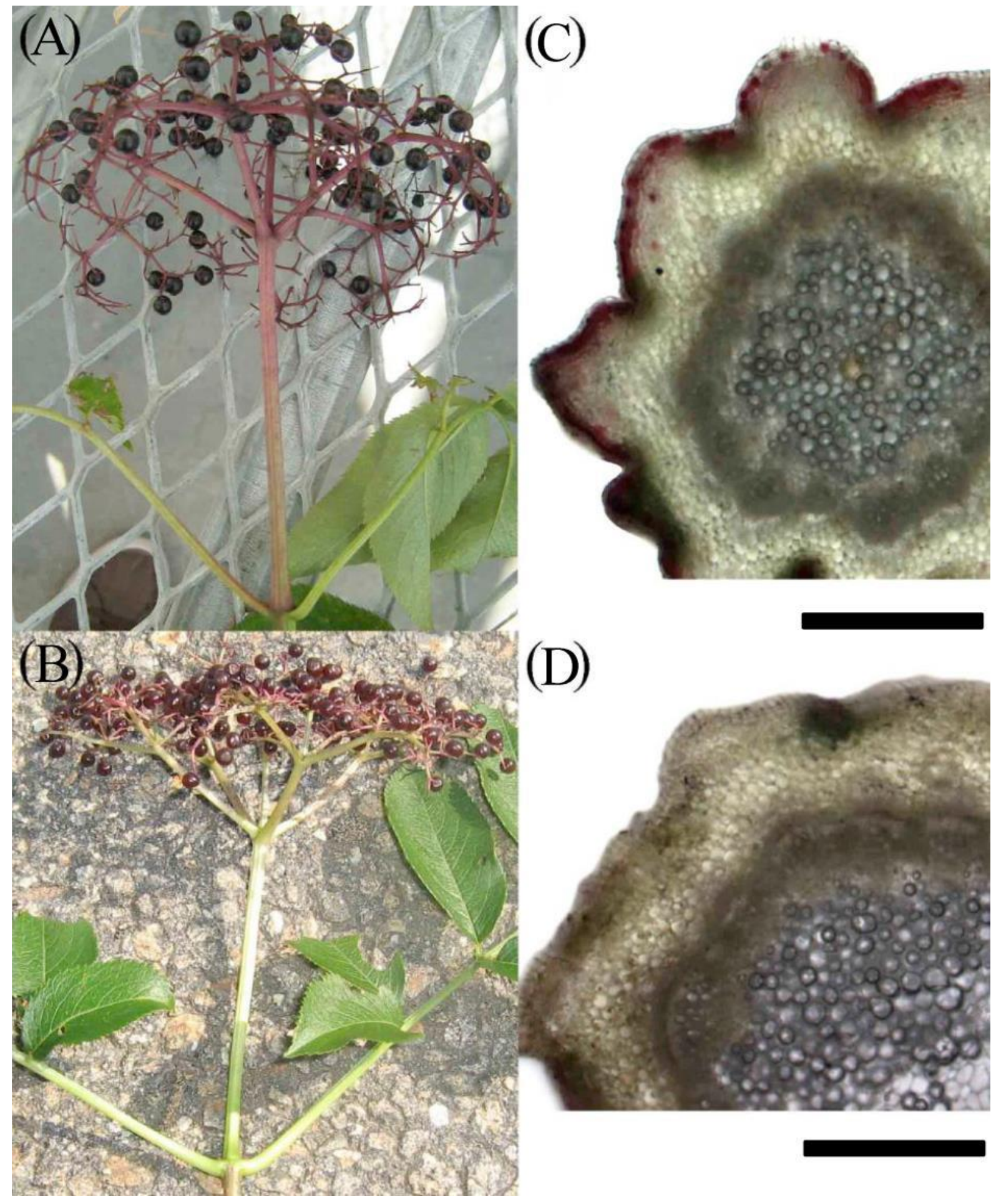

Fig 4.1. Infructescences of Sambucus canadensis. Photograph of infructescences bearing red (A) and green (B) peduncles, and photomicrographs of transverse sections through red (C) and green (D) peduncles. Bars, $1 \mathrm{~mm}$. 

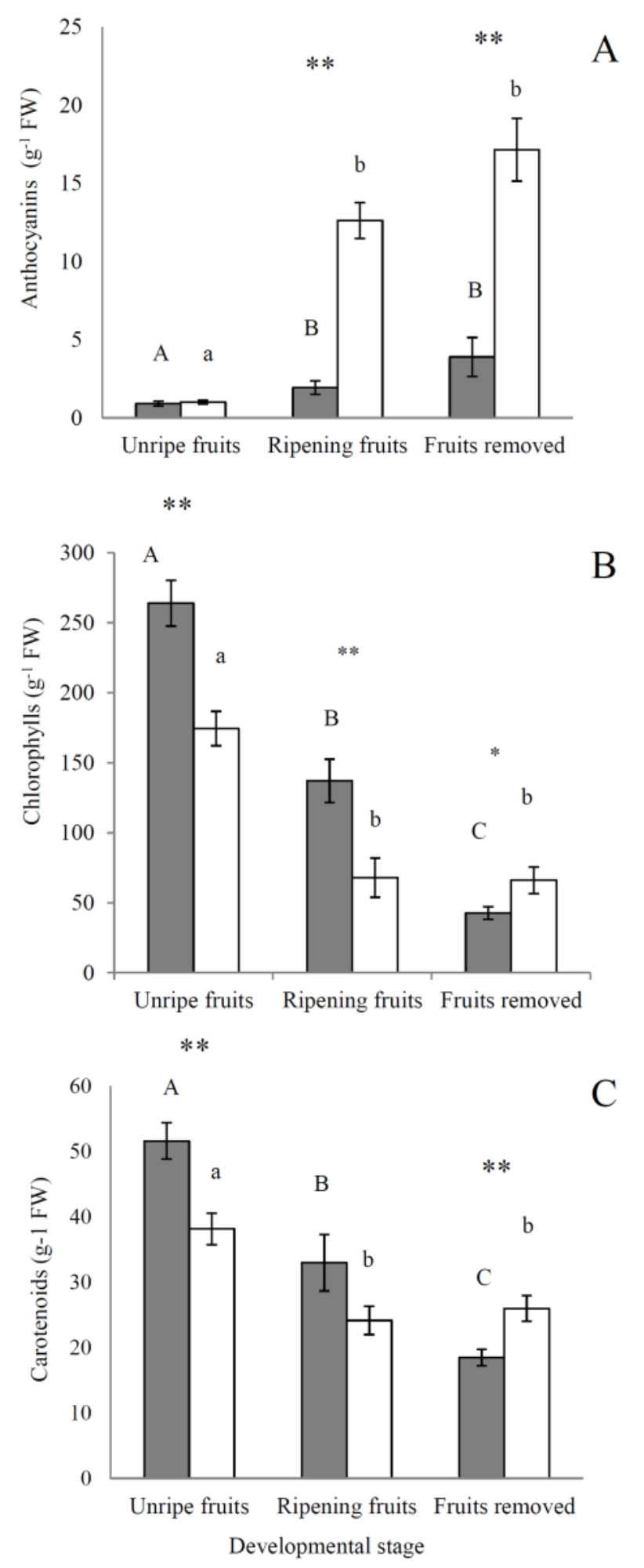

Fig 4.2. Mean $( \pm$ SE) anthocyanin (A), chlorophyll (B) and carotenoid (C) concentrations of shaded (closed bars) and exposed (open bars) Sambucus canadensis peduncles. Measurements were taken while fruits were unripe, while fruits were ripening, and after the fruits had been removed. Letters above bars indicate significant difference between rounds (P $<0.001)$. Statistically significant difference between peduncle types: $* P<0.05 ; * * P<0.01$. 
Peduncles that were artificially shaded with neutral density polycarbonate filters while fruits were still unripe, contained anthocyanin concentrations that were $23 \%$ those of peduncles onto which a transparent light filter was applied, and 19\% those of naturally exposed peduncles $(P<0.02)$. No difference was found in anthocyanin concentrations between those peduncles on which a transparent light filter was applied and exposed peduncles on which no filter was applied $(P=0.5$; Table 4.1$)$.

Table 4.1. Mean $( \pm \mathrm{SE})$ pigment concentrations from Sambucus canadensis peduncles that were either exposed, covered by transparent light filter or a neutral density light filter. $n=8$.

\section{Treatment}

\begin{tabular}{llll}
\hline $\mathrm{g}^{-1} \mathrm{FW}$ & Exposed & Transparent filter & Neutral density filter \\
\hline Chlorophyll a & $36.8(6.2)$ & $24.2(5.5)$ & $48.2(7.5)$ \\
Chlorophyll b & $21.3(4.0)$ & $16.7(2.1)$ & $21.2(3.0)$ \\
Chl a:b & $1.8(0.2)$ & $1.4(0.2)$ & $2.3(0.2)$ \\
Total chlorophyll & $58.2(9.7)$ & $40.8(7.4)$ & $69.3(10.2)$ \\
Total anthocyanins & $8.7(2.3)$ & $6.8(1.3)$ & $1.6(0.2)$ \\
Total carotenoids & $26.5(2.7)$ & $19.0(2.0)$ & $22.8(2.0)$ \\
\hline
\end{tabular}

\subsubsection{Parameters of photosynthetic health before, during, and after fruit maturation}

When fruits were still unripe and both sun and shade acclimated peduncles were green, values of dark-acclimated $\mathrm{Fv} / \mathrm{Fm}$ were significantly higher for shade-acclimated peduncles compared to sun-acclimated peduncles. When exposed to an irradiance ramp, peaking at 1600 $\mu$ mol photons $\mathrm{m}^{-2} \mathrm{~s}^{-1}$ of white light, sun acclimated peduncles exhibited small, but significantly higher quantum efficiencies $\left(\Phi_{P S I I}\right)$ than did shade acclimated peduncles (sunacclimated, $0.05 \pm 0.005$; shade-acclimated, $0.03 \pm 0.002 ; P<0.05$; Fig 4.3 ) for the first two hours of treatment, but were comparable for the subsequent hour and during the recovery of $F v / F m$ when returned to the dark (Fig 4.3). In contrast, after 80 min of $1600 \mu \mathrm{mol}$ photons $\mathrm{m}^{-}$ 
${ }^{2} \mathrm{~s}^{-1}$ of white light sun-acclimated peduncles showed levels of non-photochemical quenching $(N P Q)$ that were $40 \%$ less than that of shade-acclimated peduncles (Fig 4.3).

During the second round of measurements taken when fruits were ripening, values of darkacclimated $F v / F m$ were comparable between shade-acclimated, green peduncles and sunacclimated, red peduncles. When exposed to a three-hour irradiance ramp, peaking at 1600 $\mu \mathrm{mol}$ photons $\mathrm{m}^{-2} \mathrm{~s}^{-1}$ of white light, $\Phi_{P S I I}$ was up to $150 \%$ higher in red peduncles compared to green peduncles while $N P Q$ was less than half that of green peduncles ( $\mathrm{P}<0.05$; Fig 4.3). When returned to the dark, no difference in $F v / F m$ recovery $(\Delta F v / F m)$ was found between red and green peduncles. During this round, an error in measuring beam intensity rendered $N P Q$ incalculable for one red replicate.

During the final round of measurements, taken after fruits had been removed, exposure to a three-hour irradiance ramp, peaking at $1600 \mu \mathrm{mol}$ photons $\mathrm{m}^{-2} \mathrm{~s}^{-1}$ of white light, revealed no difference in $\Phi_{P S I I}$ between red, sun-acclimated and green, shade-acclimated peduncles (Fig 4.3). However, green peduncles still displayed up to $62 \%$ higher $N P Q$ than red peduncles (P $<0.05$; Fig 4.3). Following the light treatment, $\Delta F v / F m$ for red peduncles $(0.18 \pm 0.02)$ was comparable to that measured during round two $(0.2 \pm 0.02)$. In contrast, Fv/Fm did not recover as completely for green peduncles during the third round of measurements $(0.3 \pm 0.3)$ compared to the extent of $F v / F m$ recovery measured during round two $(0.2 \pm 0.3 ; P<0.01)$. Consequently, red peduncles exhibited a $33 \%$ greater $F v / F m$ recovery than did green peduncles during this final round $(\mathrm{P}<0.01$; Fig 4.3). During this round of measurements, two red replicates were knocked during the treatment period, and another returned a fluorescence signal too low for analysis. This reduced the number of replicates to 7 red peduncles and 10 green peduncles. 

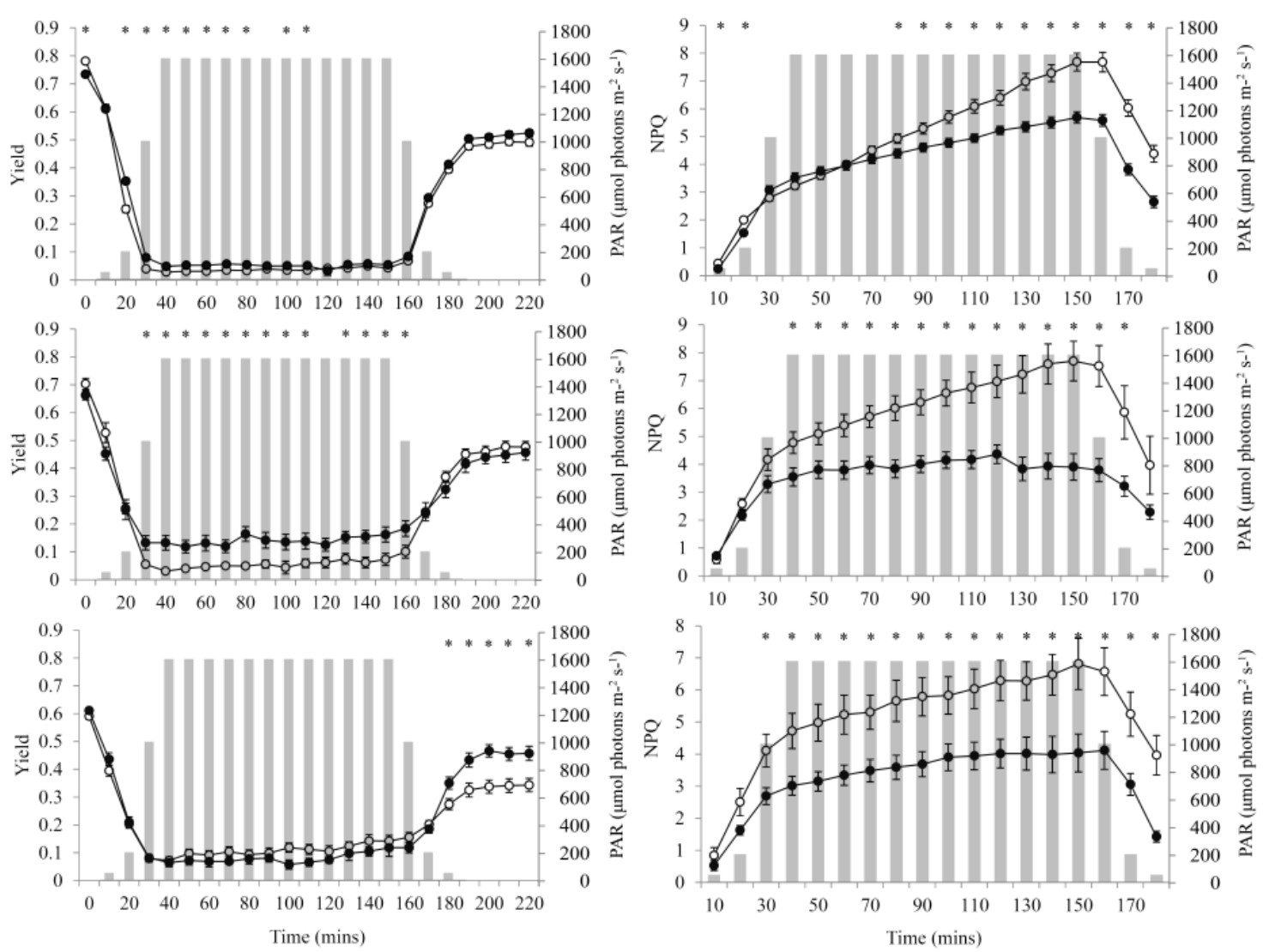

Fig 4.3. Light response curves for photochemical quantum yield $\left(\Phi_{P S I I}\right)$ and nonphotochemical quenching (NPQ) for sun-acclimated (•) and shade-acclimated (०) peduncles before (top), during (middle) and after fruit ripening (below). Shade-acclimated peduncles were green at all three ontogenetic stages while sun-acclimated peduncles were green during the first stage and red for the subsequent two stages. Means \pm SE, * indicates statistically significant differences between groups. Shaded bars represent PAR for each time point.

\subsubsection{Comparative rates of xanthophyll de-epoxidation}

For those peduncles from which xanthophyll pigments were quantified, the ratio of xanthophyll to chlorophyll was $165 \%$ higher in green peduncles compared to red $(P<0.01$; Fig 4.4). Following a 10 min treatment of $1600 \mu \mathrm{mol}$ photons $\mathrm{m}^{-2} \mathrm{~s}^{-1}$ of white light, green peduncles also showed a $38 \%$ higher rate of xanthophyll cycle de-epoxidation compared to red peduncles, exhibiting a higher proportion of zeaxanthan and antheraxanthan to the total xanthophyll pool $(P<0.001$; Fig 4.4). During this 10 min treatment period, green peduncles also exhibited levels of $N P Q$ that were $17 \%$ higher than those of red peduncles $\left(F_{1,18}=5.1\right.$, $P=0.04)$. 

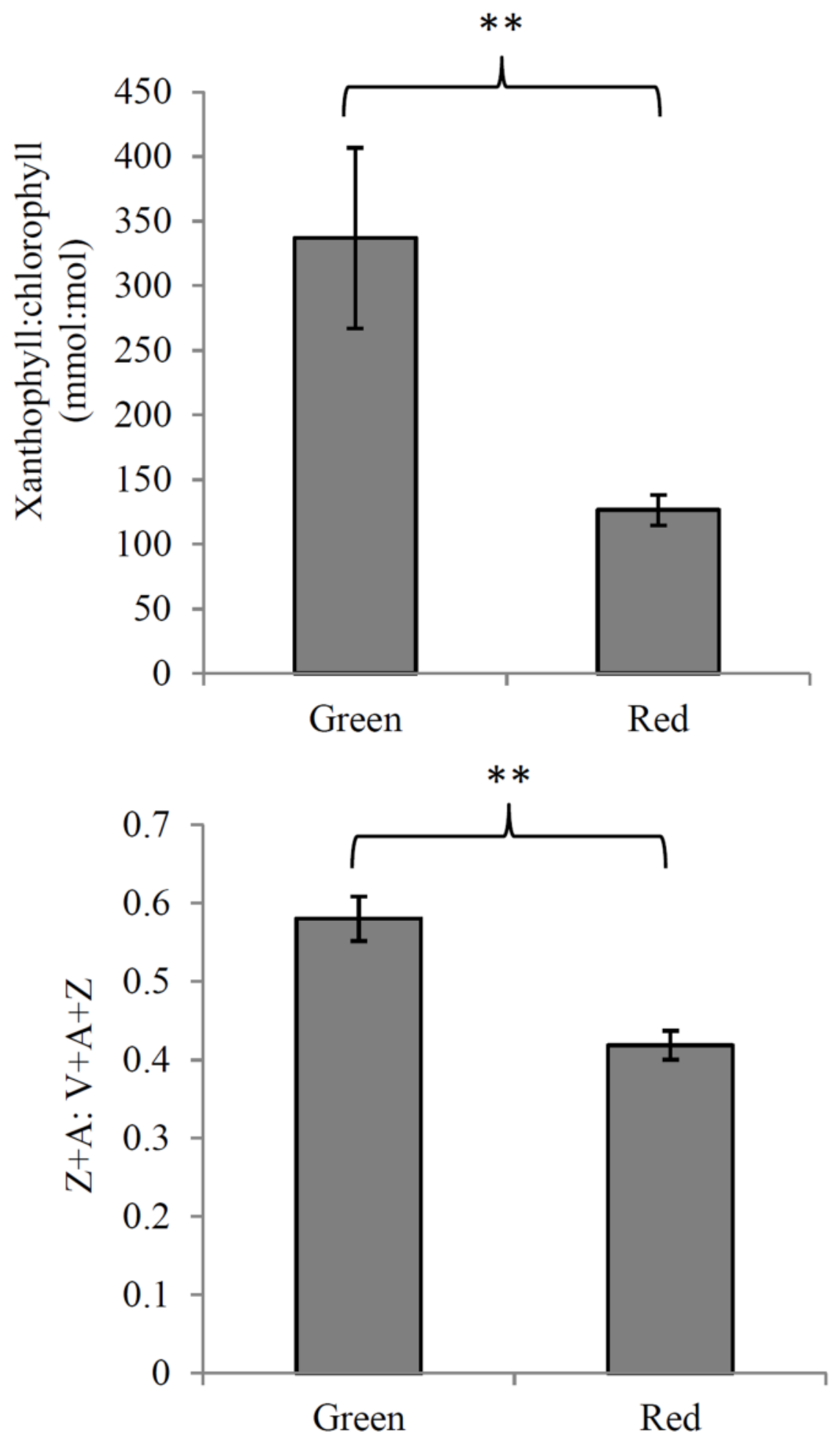

Peduncle colour

Fig 4.4. Mean $( \pm \mathrm{SE})$ ratios of xanthophyll to chlorophyll concentrations (top) and deepoxidized xanthophylls, zeaxanthin and antheraxanthin, to total xanthophyll concentrations (bottom) for red and green Sambucus canadensis peduncles. **, $P<0.01$. 


\subsubsection{Light screening experiment}

Green peduncles manipulated with either a simulated anthocyanic light filter, or a transparent light filter, took between 4 and 65 days to abscise after the plants on which they were borne were relocated into a high light environment. No difference in longevity (Fig 4.5), lipid peroxidation (as estimated by TBARS content), or nitrogen concentration (Fig 4.6) were found between the infructescences on those two sets of plants $(P>0.9)$. There was no relationship between $\% \mathrm{~N}$ in an abscised peduncle and the number of days it took to abscise $\left(F_{1,19}=0.01, P=0.9\right)$. Pre-dawn, variable to maximum chlorophyll fluorescence measurements $(F v / F m)$ were also comparable between those infructescences bearing a transparent light filter, and those bearing a fake anthocyanic light filter. Sigmoidal regressions, calculated for each peduncle, and describing the initial value of $F v / F m$, the duration this initial $F v / F m$ was maintained, and the rate at which $F v / F m$ declined, showed that none of the three parameters differed between treatments $(\mathrm{P}>0.3)$.

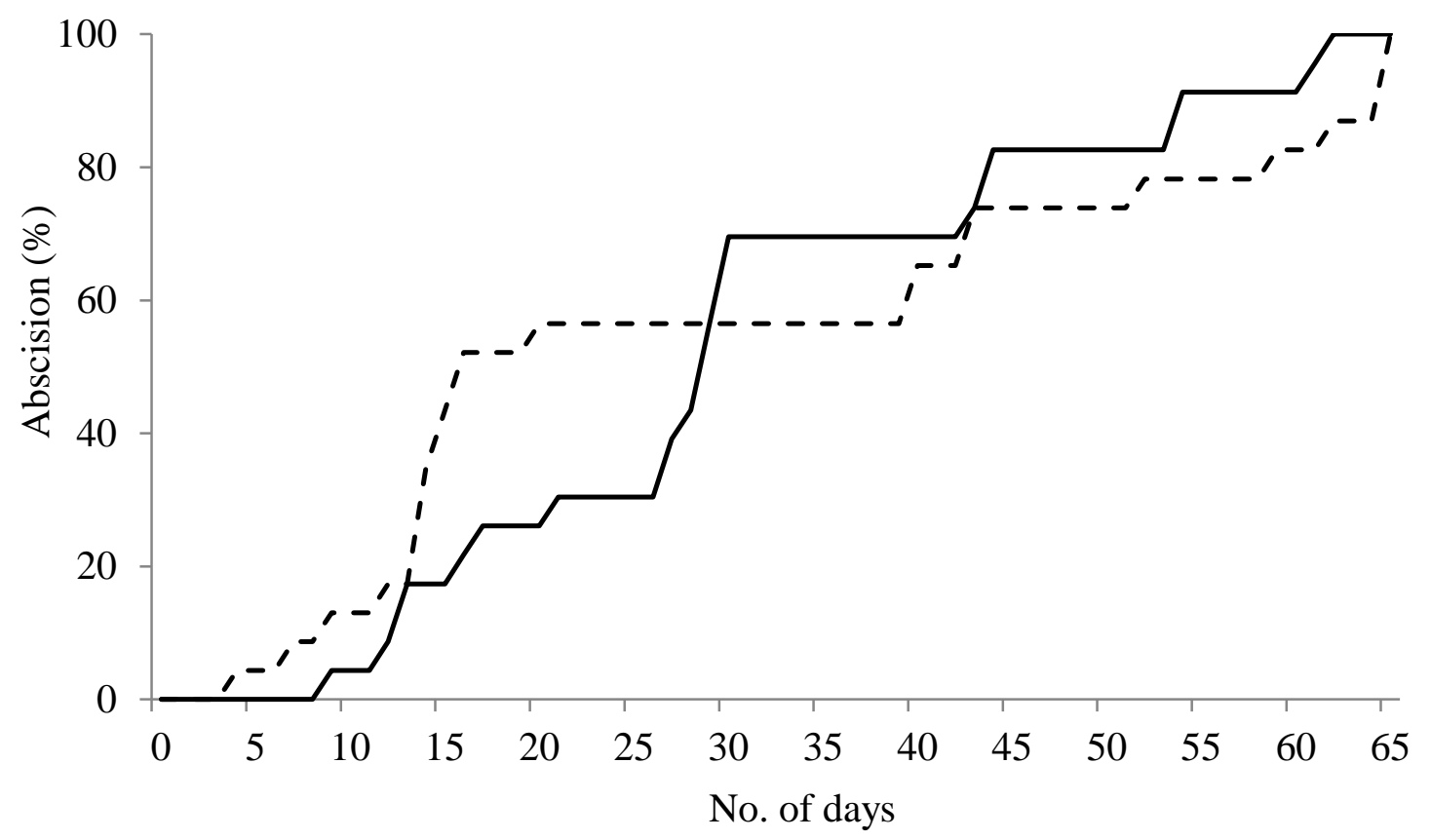

Fig 4.5. Proportions of green Sambucus canadensis infructescences which abscised after peduncles had been covered with either a red filter (solid line) or transparent light filter (dotted line). $n=23$. 

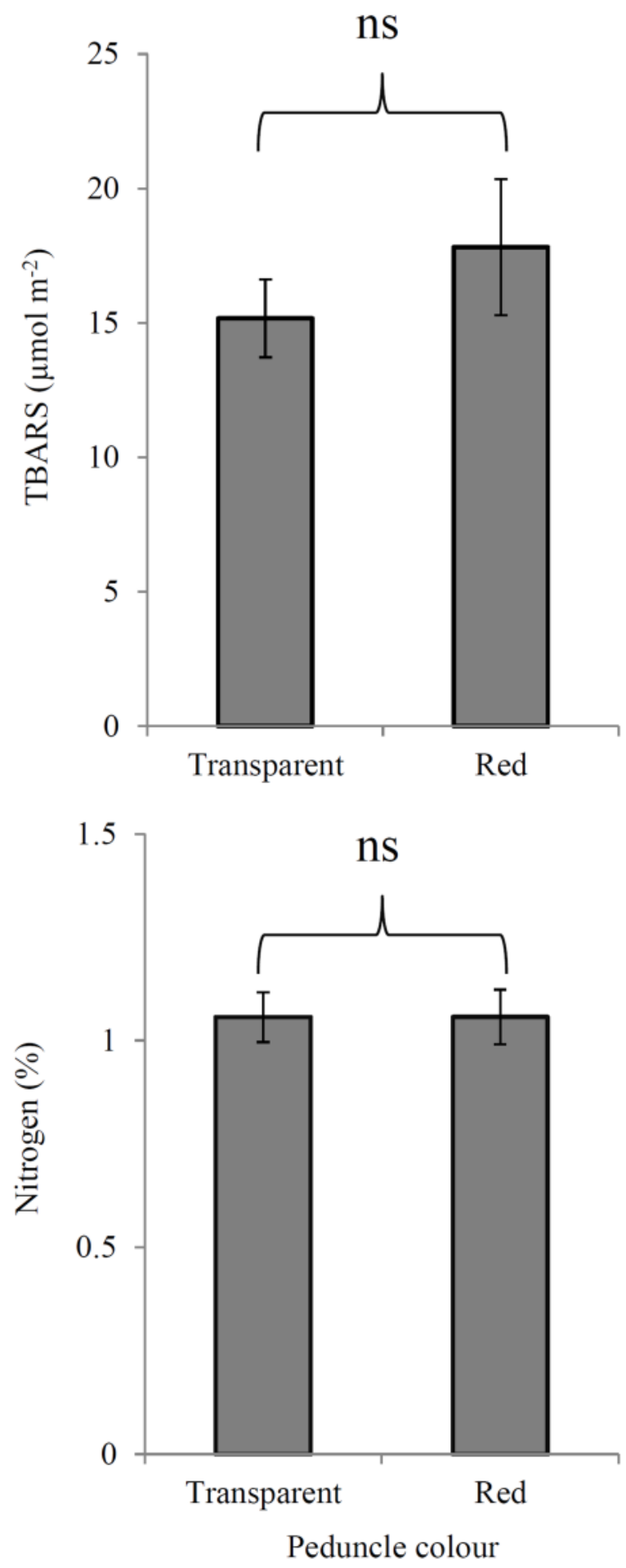

Fig 4.6. Mean $( \pm S E)$ content of thiobarbituric acid-reactive substances (TBARS; above) and nitrogen (below) from green Sambucus canadensis rays and peduncles (respectively), each bearing either a transparent, or a red, anthocyanic light filter. Content of TBARS was quantified from rays collected in full midday sun $(n=13)$, while nitrogen was quantified from peduncles following senescence $(n=21)$. 


\subsubsection{Tensile strength}

For infructescences collected after fruits had been naturally removed, red peduncles were both stronger and more elastic than green peduncles $(P<0.001)$, requiring twice the force and extending almost $80 \%$ longer (Fig 4.7) before breaking. The red peduncles were also thinner, displaying a cross-sectional area on average $68 \%$ that of green peduncles, but this attribute could not account for the difference in tensile strength of the two phenotypes, i.e. cross sectional area did not correlate with either breaking force $\left(F_{1,32}=0.05, P=0.8\right)$ or breaking extension $\left(F_{1,32}=1.2, P=0.3\right)$. Three green and three red samples broke at the attachment site to the tensile strength meter and were omitted from this analysis. This reduced the number of replicates to 17 for each phenotype. 

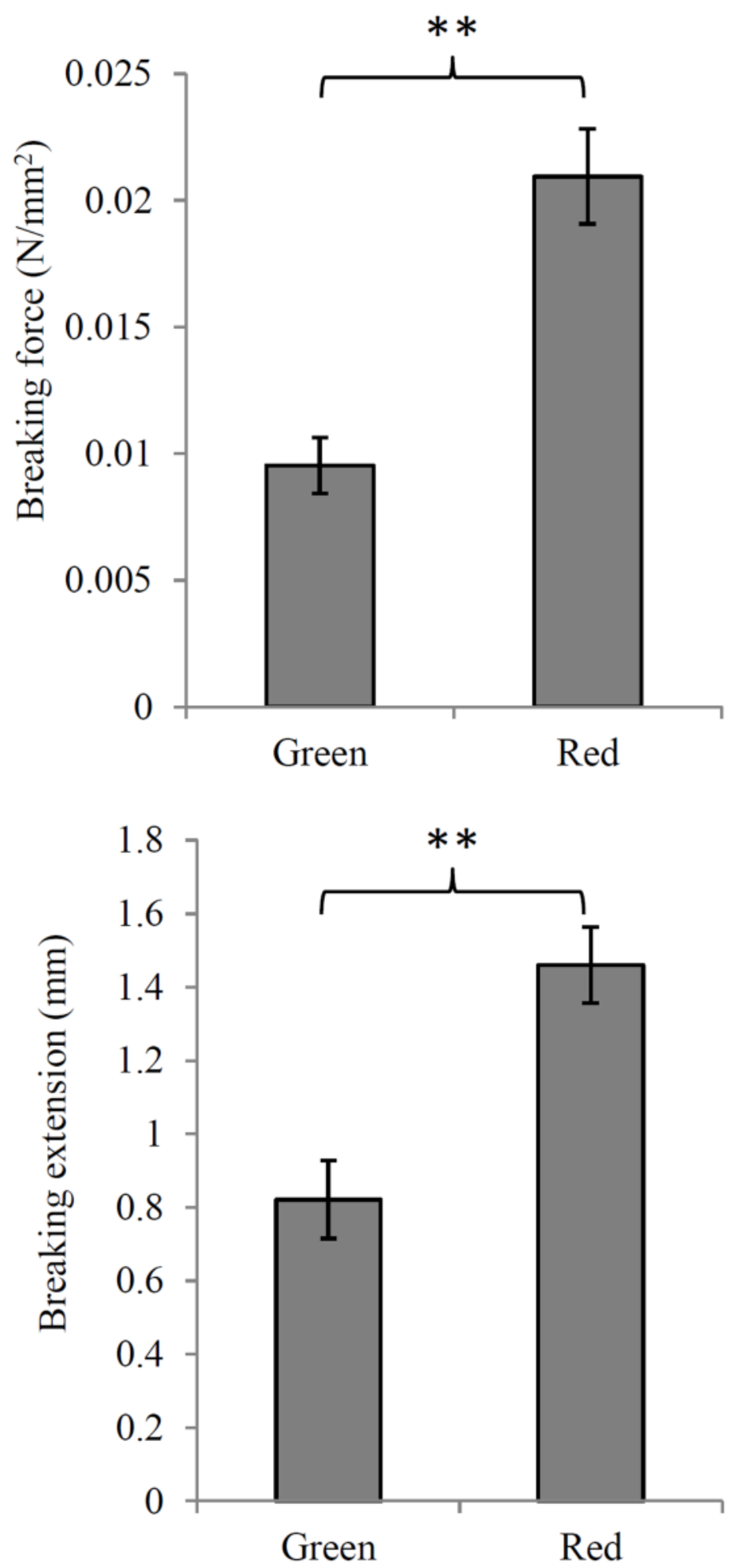

Peduncle colour

Fig 4.7. Mean ( \pm SE) breaking force (top) and breaking extension (bottom) of red and green Sambucus canadensis peduncles. ${ }^{*}, P<0.001$. 


\subsection{Discussion}

This study provides some support for the hypothesis: 'anthocyanin accumulation prolongs senescence in Sambucus canadensis peduncles'. Firstly, I found that both chlorophyll and carotenoid concentrations decreased more rapidly in senescing green peduncles compared to senescing red peduncles (Fig 4.2), and that in the later stages of senescence, tensile strength and elasticity were significantly higher in red peduncles, compared to green (Fig 4.7). I also found that a peduncle's ability to mitigate light stress diminished more quickly in green peduncles compared to red (Fig 4.3). Following light stress, red peduncles collected during the later stages of senescence showed a drop in quantum yield $(\mathrm{Fv} / \mathrm{Fm})$ comparable to that displayed by red infructescences collected while fruits were ripening. In contrast, green peduncles collected during the later stages of senescence displayed a significantly higher decline in $\mathrm{Fv} / \mathrm{Fm}$ following light stress, than did green peduncles collected while fruits were ripening (Fig 4.3). However, these results are incongruous with our long-term experiment manipulating peduncle colour with an artificial anthocyanic light filter. Neither pre-dawn quantum yield, nitrogen concentrations following abscission, lipid peroxidation, nor infructescence longevity differed between green infructescences bearing transparent light filters, and green peduncles bearing artificial anthocyanic light filters. Chapter 3 showed that the light-screening properties of anthocyanins are directly responsible for mitigating light stress in red peduncles of $S$. nigra. In this study, I demonstrated that the red peduncles of $S$. canadensis were similarly equipped to avoid light stress at the latter stages of senescence, and while the addition of an artificial anthocyanic layer did not prolong senescence and did not enhance nitrogen resorption, I have shown that red peduncles possess several attributes indicative of a prolonged senescence.

\subsubsection{The similarities between red senescing peduncles and red senescing leaves}

This study showed that many of the attributes associated with prolonged senescence, as demonstrated for leaves, are similarly present in red senescing peduncles. Schaberg et al. (2008) showed that the retention strength of senescing anthocyanic leaves was significantly greater than that of senescing acyanic leaves. Here, green peduncles were likewise, more weakly attached to the plant, breaking under half the force required to break red peduncles (Fig 4.7). Senescence in a given organ is followed by abscission and progression of an abscission layer, in this case estimated by tensile strength, provides a useful means of comparing the stages of senescence (dela Fuente \& Leopold, 1968). Those green peduncles 
that were more weakly attached to the plant were presumably at a later stage of senescence, relative to red peduncles. Moreover, red peduncles were more elastic than green peduncles (Fig 4.7), a trait which for petioles at least, decreases as tissues age (Chazdon, 1986). The infructescences collected for this experiment were from plants in a single road-side clearing, thus wind exposure (and an infructescence's acclimation to that exposure) was assumed to be comparable for all infructescences. For this reason, we suggest that differences in tensile strength and elasticity are likely attributable to differences in the rate of progression of senescence.

Schaberg et al.'s (2008) study found significantly higher carotenoid concentrations in senescing anthocyanic leaves compared to senescing acyanic leaves. Here, red S. canadensis peduncles similarly displayed higher carotenoid concentrations towards the final stages of senescence (Fig 4.2). Moreover, these data show that prior to senescence, green peduncles actually contained higher concentrations of carotenoids than did red. Evidently, the difference between red and green peduncles at the later stages of senescence is because carotenoid concentrations decreased more quickly in green peduncles compared to red. Similarly, our data show that the decline in chlorophyll concentrations occurred more quickly in green peduncles (Fig 4.2). Like carotenoids, chlorophyll concentrations were greater in green peduncles during the early stages of senescence, but greater in the red peduncles during the later stages of senescence. Evidently, much of the indirect support for a prolonged senescence for red leaves is similarly present in red peduncles.

\subsubsection{Complimentary light screening mechanisms in $\mathrm{S}$. canadensis peduncles}

In this study, we found that light stress in S. canadensis peduncles is reduced by both the light screening properties of anthocyanins and by thermal energy dissipation via the xanthophyll cycle. Compared to red peduncles, green peduncles contained higher concentrations of total carotenoids (Fig 4.2) and when exposed to high light intensities, displayed higher levels of non-photochemical quenching and xanthophyll de-epoxidation (Fig 4.3; Fig 4.4). These phenotypes evidently use differing strategies to avoid photoinhibition and, during the early stages of senescence maintain comparable levels of photoinactivation $(\triangle F v / F m)$ following light stress (Fig 4.3). However, the decline in total carotenoids (Fig 4.2) reduced each phenotype's capacity to dissipate light energy via the xanthophyll cycle (as estimated by a reduction in non-photochemical quenching; Fig 4.3). Not surprisingly, during these later stages of senescence the severity in photoinactivation $(\Delta F v / F m)$ was significantly 
higher in green peduncles compared to red (Fig 4.3). While data described in chapter 3, as well as those of Field et al. (2001) and Hoch et al. (2003) showed improved recovery of photosynthetic yield for red senescing tissue compared to green, this study additionally showed that anthocyanin accumulation provides a means of photoprotection which, unlike the xanthophyll cycle, is maintained during senescence.

\subsubsection{Why might anthocyanin accumulation prolong senescence?}

The mechanism by which anthocyanin accumulation prolongs senescence is unknown; however, the photoprotective properties of anthocyanins have been implicated. Ethylene and auxin are among the hormones responsible for coordinating senescence in both leaves and peduncles, with ethylene accelerating and auxin retarding the process (Lyons \& Widmer, 1984; Goszczynska \& Zieslin, 1993; Roberts et al., 2000; Roberts et al., 2002; Schaberg et $a l ., 2008)$. There is some evidence to suggest that ROS increases a tissue's sensitivity to ethylene while simultaneously reducing auxin transport, thereby hastening senescence (Michaeli et al., 1999a; Michaeli et al., 1999b). By screening peduncles from excess light, anthocyanins may prevent photoinhibition, reduce the production of ROS and prolong the senescence period. Alternatively, oxidative stress may hasten senescence via a more direct process. ROS are responsible for pigment, protein and lipid oxidation during senescence; essential for subsequent nutrient remobilization (Hörtensteiner \& Feller, 2002; Juvany et al., 2013). Thereafter, oxidative processes are among the mechanisms that result in cell death (Zimmermann \& Zentgraf, 2005). If produced in excess during senescence's phase of nutrient mobilization, ROS may initiate this terminus phase prematurely (Juvany et al., 2013). Once again, anthocyanins may prolong senescence by preventing photoinhibition and the excess production of ROS.

This study was the first to test whether the presence of an anthocyanic light screen reduces TBARS in senescing tissues. TBARS are a by-product of lipid peroxidation, and for that reason may provide a measure of oxidative damage. However, I found no difference in TBARS content between $S$. canadensis rays which bore an anthocyanic light filter, and rays which bore a transparent light filter (Fig 4.6). This result suggests that an anthocyanic light screen either, does not reduce ROS production, or if it does, the difference in ROS production between treatments was not in excess of the antioxidant mechanisms $S$. canadensis rays possess. Alternatively it may be that this experimental design failed to isolate the conditions under which anthocyanins reduce lipid peroxidation (see section 4.5.4 for further discussion). 
Anthocyanins are potent antioxidants themselves (Wang et al., 1997) and this design excludes the potential for them to scavenge ROS directly. This limitation is highlighted by Zhang et al. (2012) who showed for leaves of Arabidopsis thaliana, that the presence of anthocyanins, even in concentrations too low to be an effective light screen, coincides with a reduction in photo-oxidative damage. It may be that anthocyanins reduce oxidative damage in S. canadensis, but do so by scavenging ROS directly, not by acting as a light screen. Regardless, additional studies are needed if we are to determine the mechanism (if one exists) by which anthocyanins prolong senescence.

\subsubsection{Are anthocyanins responsible for a prolonged senescence?}

It is unsurprising that the mechanism by which anthocyanins prolong senescence has yet to be identified, given that support for a prolonged senescence remains indirect (Schaberg et al., 2008; Zhang et al., 2013b); we cannot examine why anthocyanins prolong senescence without first showing that anthocyanins are responsible for prolonging senescence. This study is the first to manipulate the profiles of transmitted light to senescing tissue, thereby isolating the effect of anthocyanins, and then compare longevity, TBARS content, and nitrogen resorption. However, we found no difference in longevity (Fig 4.5), lipid peroxidation or nitrogen resorption (Fig 4.6) between green peduncles bearing an artificial anthocyanic light filter, and green peduncles bearing a transparent light filter. This suggests that either anthocyanins do not promote peduncle longevity, or as discussed above, my experimental design failed to replicate the conditions under which anthocyanins prolong peduncle longevity. A simple observation might explain the latter scenario. Fruit removal was high for those relocated plants, with nearly $100 \%$ of fruits consumed. It is likely that senescence is accelerated in peduncles after fruits are removed, and this overrides any prolonging of senescence anthocyanins may impart. Alternatively, the plants used in the longevity experiment were shade-acclimated, and the entire plant may have been light stressed when relocated. Light stressing the entire plant may have overridden potential benefits of light screening in the peduncle. Finally, it may be that anthocyanins prolong senescence by some means other than light abatement, for example, as discussed in section 4.5.3, by directly scavenging ROS. I acknowledge the limitations in this experimental design, and suggest it might explain why the results from this experiment were incongruous with the rest of my study, which are consistent with a prolonged senescence attributable to red peduncles. 


\subsection{Conclusion}

Compared to red peduncles, green peduncles of $S$. canadensis possess several attributes indicative of tissue nearing the latter stages of senescence. However, experimentally manipulating green peduncles, adding to them a fake anthocyanic layer, did not enhance longevity or nutrient resorption. Thus, anthocyanins are responsible for a reduction in light stress, and red peduncles display characteristics indicative of a prolonged senescence, however, I was unable to demonstrate that the light screening properties of anthocyanins are directly responsible. Regardless, my data highlight the importance of anthocyanin accumulation during a period when other ameliorators of light stress fail. Furthermore, I show that a prolonged senescence, as described for anthocyanic leaves, may similarly be present in reproductive organs. 


\section{Chapter 5: General Discussion}

\subsection{Introduction}

Anthocyanins perform both physiological and communicative functions in plants, yet there is a dearth of knowledge on physiological functions in reproductive organs and on communicative functions in vegetative organs. While a number of 'communication' hypotheses have been proposed for anthocyanic colouration in vegetative organs, direct support had until recently been lacking. Conversely, some of the physiological functions proposed for anthocyanins have been investigated in reproductive organs, though exclusively in the fruits of cultivated varieties. These omissions in anthocyanin research must be redressed if we are to understand the full assemblage of functions that are performed by anthocyanins in vegetative and reproductive organs.

\subsection{Novel discoveries from this thesis}

This study advances our understanding of the role of anthocyanins in plants in two ways: it is the first to directly demonstrate that anthocyanins perform a communicative function in vegetative organs, and is the first to show for a naturalised (non-cultivar) species, that anthocyanins perform a physiological function in reproductive organs. Chapter two showed that the red (anthocyanic) leaf margins in Pseudowintera colorata provided information on a leaf's defensive investment and in a natural population, corresponded to a reduction in herbivory. Moreover, it was demonstrated that anthocyanic colouration per se, rather than possible olfactory cues, is the trait by which a herbivore discriminates between highlydefended and poorly-defended leaves. I showed that a natural P. colorata herbivore, Ctenopseustis obliquana, preferred green-margined leaves to red, but only under white light. When the light environment was manipulated such that leaf colour discrimination was impaired, red- and green-margined leaves were consumed equally.

Chapter three demonstrated that anthocyanins in the peduncles of Sambucus nigra provide a photoprotective function, screening photosynthetic tissues from the detrimental effects of excess light. I showed that anthocyanins were responsible for absorbing a significant proportion of green light that would otherwise be intercepted by a peduncle's mesophyll tissue and during high-light exposure, red peduncles maintained a higher photosynthetic efficiency than did green peduncles. Following light stress, red peduncles were also less 
photo-inactivated than green peduncles. Most importantly, I showed that the differences in photoinhibition between red and green peduncles were attributable to the light screening properties of anthocyanins. For variegated red/green peduncles, the green basal portions displayed a higher level of photoinactivation than did red apical portions. In contrast, the base and apex were equally photoinhibited in peduncles that were entirely green. Finally, by testing the requirements of a communicative function previously described for anthocyanins in S. nigra peduncles, I found little evidence to support anthocyanin's role in frugivore attraction, previously described by Schaefer and Braun (2009). Anthocyanin concentrations in the peduncle were a reliable indicator of fruit quality, yet reducing fruit sugar content did not reduce peduncle anthocyanins. Moreover, I found no relationship between peduncle colour and frugivory when fruit removal was quantified for the duration of a fruiting season.

In chapter four, I showed that red Sambucus canadensis peduncles displayed several characteristics indicative of a prolonged senescence, relative to green peduncles. Chlorophyll and xanthophyll catabolised more quickly in green peduncles compared to red, and at the later stages of senescence, abscission layer progression, as estimated by peduncle retention strength, had progressed farther in green peduncles compared to red. I also found that a peduncle's ability to ameliorate the effects of light stress declined more quickly in green peduncles compared to red. While both displayed comparable levels of photoinhibition when light stressed at the early stages of senescence, at the later stage of senescence, green peduncles displayed significantly higher levels of photoinhibition than did red peduncles following light stress. Finally, to simulate the effect of anthocyanins on senescing tissue, I created a novel experiment wherein green peduncles senesced while shaded by an artificial light filter, the absorbance properties of which were comparable to anthocyanins. In contrast to the data above which provided indirect support for a prolonged senescence, the addition of an artificial anthocyanic screen did not prolong senescence. The length of time peduncles wrapped in anthocyanic light filters took to senesce was comparable to adjacent peduncles bearing transparent light filters. Similarly, I found no evidence to suggest that anthocyanins improve nitrogen resorption in senescing $S$. canadensis peduncles, a function proposed for anthocyanins in senescing leaves. 


\subsection{The significance of identifying a communicative function for red $\mathrm{P}$. colorata leaf margins}

My study identified a plant-herbivore relationship, previously undiscovered among plants; it is the first to show unequivocally that foliar reddening can provide information on leaf defences (section 2.4.2), and that approaching herbivores can use that information when selecting leaves (section 2.4.4). A number of hypotheses implicate a signalling role for leaf reddening and of considerable interest to my data are 'coevolution' (Archetti, 2000; Hamilton \& Brown, 2001), 'aposematism' (Poulton, 1890), 'defence indication' (Schaefer \& Rolshausen, 2006) and 'photoprotection of photo-labile defence compounds' (Page \& Towers, 2002); those which assume a correlation between leaf reddening and chemical defences. For each hypothesis, the information that anthocyanic colouration provides is comparable (i.e. reddening signifies increased leaf defences), yet each hypothesis makes a unique prediction about how this correlation may have evolved. The last decade has seen numerous publications debating the predictions and potential significance of each hypothesis, yet until now, a communicative function for foliar anthocyanins had not been demonstrated. Below I will discuss the significance of my data with regard to each of these hypotheses.

\subsubsection{P. colorata leaf margins and coevolution}

Of those hypotheses implicating a signalling role for anthocyanins in vegetative tissues, Archetti's (2000) and Hamilton and Brown's (2001) coevolution hypothesis has arguably received the most attention. Coevolution proposes that leaf reddening serves as a handicap signal, a costly extravagance which only the healthiest, best-defended plants can employ. This hypothesis makes three predictions with regard to anthocyanic leaves:

1. red leaves are chemically better defended

2. herbivores avoid redder leaves

3. signalling leaf palatability provides a selective advantage

Until I completed the research described in chapter 2, no study had fulfilled any of the three requirements for coevolution. The first requirement had received indirect support from a number of studies (see section 1.4.4), but none had shown that anthocyanic colouration corresponded to any level of defensive investment. By utilising P. colorata to test the first requirement of coevolution, I showed that the presence of red (anthocyanic) leaf margins 
corresponded to a specific compound, polygodial, which is known to deter herbivory (Asakawa et al., 1988). For P. colorata, red-margined leaves contained significantly higher polygodial concentrations in both the leaf margin and leaf interior than leaves with green margins (Fig 2.6). Following publication of these data (Cooney et al., 2012), Menzies (2013) presented a comparable analysis in his doctoral thesis, utilizing a population of $P$. colorata which exhibits a different pattern of leaf reddening (anthocyanins were not restricted to the margin, but accumulated across the entire lamina). Menzies' (2013) data similarly showed a positive relationship between polygodial concentrations and the extent of foliar reddening in $P$. colorata, and in combination with my data, we showed that the positive relationship between anthocyanic reddening and polygodial in $P$. colorata leaves fulfils the first requirement of the coevolution hypothesis.

The second prediction of the coevolution hypothesis, that 'leaf colour alters herbivore behaviour' had similarly received only indirect support. Numerous studies had demonstrated a preference for green leaves over red (Furuta, 1986; Archetti \& Leather, 2005; Rolshausen \& Schaefer, 2007; Wong \& Srivastava, 2010), and Doring et al. (2009) had shown that that among differently coloured traps, aphid visitation was lower when traps were painted red. However, no study had demonstrated that a preference for green leaves is specifically attributable to leaf colour (as opposed to olfactory cues) and Doring et al.'s (2009) study did not compare visitation rates for leaves. In my study, I isolated the effect of leaf colour on herbivore behaviour and showed that margin colour is the trait by which $C$. obliquana larvae discriminate between red- and green-margined $P$. colorata leaves. By identifying this, my study is the first to fulfil the second requirement of the coevolution hypothesis.

Subsequent to the publication of thesis chapter two, the second requirement for coevolution has received additional support from a study which replicated my experimental design. Using red and green Malus domestica (apple) leaves, Markwick et al. (2013) showed that larvae of the light-brown apple moth, Epiphyas postvittana preferred green leaves to red, but only under white light when discrimination of leaf colour is possible. When leaves were presented in the dark, preventing discrimination of leaf colour, this preference for green leaves disappeared. Evidently, anthocyanic colouration was responsible for imparting a communicative function in a species other than P. colorata.

The third prediction of the coevolution hypothesis states that 'signalling leaf palatability provides a fitness advantage'. By fulfilling the first two predictions of the coevolution 
hypothesis, I fulfil a crucial pre-requisite to testing this third prediction i.e. one cannot test whether a communicative function provides a selective advantage without first demonstrating that a communicative function exists. Following publication of my data, Menzies (2013) compared herbivory, carbon assimilation and fitness (i.e. fruit production) between red and green $P$. colorata individuals. As mentioned above, foliar reddening in Menzies' (2013) $P$. colorata population was not restricted to the margin, but occurred across all portions of the lamina. Comparable to my data, Menzies (2013) showed that red P. colorata leaves incurred less herbivory than green, yet contrary to the third prediction of the coevolution hypothesis, Menzies (2013) found no difference in reproductive output for red and green leaved $P$. colorata. Instead, Menzies (2013) showed that carbon assimilation was significantly reduced for red leaves compared to green, potentially because the light screening properties of anthocyanins reduced the proportion of light available for photosynthesis. For this reason, Menzies (2013) suggested that there might be a trade-off between deterring herbivores and optimizing photosynthesis; red leaves deter herbivores yet have a reduced capacity for photosynthesis while green leaves incur herbivory yet maintain higher photosynthetic rates. Phenotypic differences in leaf colour may therefore represent two distinct strategies at utilizing this trade-off.

In the population of $P$. colorata I investigated, reddening was restricted to the leaf margin and was an effective herbivore deterrent when extending as little as $2 \%$ across the leaf lamina surface. Unlike anthocyanins produced across the entire lamina, marginal anthocyanins would not provide an effective light screen and the cost to photosynthetic assimilation would be negligible. Red margins may therefore provide a means of signalling a defensive investment without reducing a plant's ability to photosynthesize.

\subsubsection{P. colorata and aposematism}

An aposematic signal is one employed to promote recognition of a well-defended species (Poulton, 1890). While aposematic signals are common within the animal kingdom, several examples have been proposed for plants (Lev-Yadun, 2003b; Lev-Yadun, 2003a; Rubino \& McCarthy, 2004; Lev-Yadun, 2009b; Lev-Yadun, 2009c; Lev-Yadun et al., 2009), yet few have been tested. In Chapter 2, I showed that a preference by $C$. obliquana larvae for greenmargined $P$. colorata leaves under white light was evident only when both leaf types had been sampled (see section 2.3.5). The avoidance of red-margined leaves appears not to be innate for C. obliquana, but rather is a learnt response; gustatory or post-ingestive feedbacks 
are evidently associated with some trait specific to each leaf type. As a preference for green leaves disappeared under red or green light, or in the dark (Fig 2.9), we know larvae did not use olfactory cues or the positional differences to discriminate between the food sources. Instead leaf colour appeared to be the trait which $C$. obliquana associated with leaf quality. My study therefore showed that red (anthocyanic) leaf margins enhanced recognition of welldefended leaves, and is the first study to provide direct support for aposematism in plants.

\subsubsection{P. colorata and defence indication}

The two hypotheses discussed above, both suggest that an association between anthocyanins and leaf defences evolved in response to herbivore pressure. In contrast, the defence indication hypothesis (Schaefer \& Rolshausen, 2006) suggests that foliar anthocyanins evolved for some other reason (e.g. photoprotection), and that anthocyanin production positively correlates to defensive chemicals because both compounds share a common biosynthetic pathway. In my study, the defence compound investigated, polygodial, is a sesquiterpene dialdehyde (McCallion et al., 1982; Perry et al., 1996b; Perry \& Gould, 2010) and biochemically distinct from flavonoids, the class of compounds to which anthocyanins belong (Holton \& Cornish, 1995; Gould et al., 2009). Therefore, the two compounds do not share a common biosynthetic pathway and the defence indication hypothesis does not explain why anthocyanins and polygodial positively correlate in $P$. colorata.

\subsubsection{Protection of photo-labile defence compounds}

An alternative hypothesis which requires a positive correlation between anthocyanins and defence compounds is 'anthocyanins protect photo-labile defence compounds' (Gould, 2004). Page and Towers (2002) showed that anthocyanin pigments in leaves of Ambrosia chamissonis provide a light screen for thiarubrines, a group of light sensitive anti-herbivore toxins present within this species. Although the light sensitivity of polygodial is unknown, we can exclude this hypothesis based on the pattern of anthocyanin accumulation in P. colorata. For those leaves I investigated, polygodial was concentrated at the leaf periphery, yet was still present in the green interior. Given that the leaf interior represents a considerably larger proportion of leaf lamina than the margin, marginal anthocyanins would provide a light screen to only a small proportion of a leaf's polygodial. It is therefore unlikely that anthocyanins in $P$. colorata evolved as a photoprotective mechanism for polygodial. 


\subsection{The significance of identifying a physiological function for red Sambucus spp. peduncles}

The adaptive significance of anthocyanin accumulation in peduncles has, until now, been exclusively considered in terms of frugivore attraction. Chapter two of this thesis shows that anthocyanins in these tissues may additionally perform a physiological function, providing a light screen and reducing the severity of light stress. This function may represent a previously unconsidered selective pressure, which unlike that communicative function proposed for anthocyanins in peduncles, may be maintained without frugivore selection. For this reason, positive selection for a photoprotective function by anthocyanins may not only explain why peduncles bearing fleshy fruits turn red, but may also explain why peduncles bearing abiotically dispersed fruits turn red. Until now, no function had been proposed for anthocyanins which commonly accumulate in those tissues (Fig 1.2). Finally, chapter four of this thesis showed that anthocyanins reduce light stress while peduncle tissues senesce, a function comparable to that described for autumnal leaves. Similarly, red peduncles displayed several characteristics indicative of a prolonged senescence, once again comparable to the characteristics displayed by red leaves during senescence. It had been proposed that the accumulation of anthocyanins may be responsible for prolonging senescence, yet this hypothesis had only been applied to senescing leaves. My data show that anthocyanins may similarly impart this benefit in senescing peduncles, though it has yet to be verified experimentally.

\subsubsection{Frugivore attraction vs. photoprotection}

The aim of chapter three was to test whether a physiological function was present for anthocyanins in S. nigra peduncles, not to compare the relative fitness contributions that physiological and communicative functions make. That said, there were results from my study which were inconsistent with Schaefer and Braun's (2009) data in support of a communicative function (i.e. frugivore attraction). First, Schaefer and Braun (2009) showed that peduncle colour was a reliable indicator of adjacent fruit quality. Fruit sugar content positively correlated with anthocyanin content in red peduncles and negatively correlated with chlorophyll content in green peduncles. Until I recognised that fruit maturation coincided with senescence in these tissues, the only explanation for the aforementioned correlations was that peduncle colour evolved so as to maximise conspicuousness. I presented an alternative hypothesis, that these correlations arose because the three variables quantified 
(anthocyanins, chlorophylls and sugar) change over time, and that there was temporal variability in the infructescences that Schaefer and Braun (2009) collected. Moreover, experimentally reducing fruit sugar content had no effect on anthocyanin accumulation in adjacent peduncles (Fig 3.4). If peduncle reddening were an adaptation to advertise fruit quality, reducing fruit quality should similarly reduce peduncle reddening.

The second piece of evidence inconsistent with that of Schaefer and Braun (2009) was that I found no relationship between peduncle colour and frugivory (Section 3.4.2). In contrast, Schaefer and Braun (2009) showed that avian fruit removal was significantly higher for infructescences bearing red peduncles compared to green. While Schaefer and Braun's (2009) experimental design examined the isolated effect of peduncle colour on seed dispersal, it was completed on excised peduncles and only monitored fruit removal for four days. My study compared frugivory for infructescences still attached to the plant, and monitored frugivory for an infructescence's entire fruiting period. When these variables were included, a preference for fruit borne on red peduncles was not found. Evidently, the data from Schaefer and Braun (2009) contrasted sharply with mine and I would contest that support for a communicative function is rather weak. However, this in no way disproves the presence of a communicative function in S. nigra and it may be that anthocyanins perform both a communicative function and a physiological function in these organs.

\subsubsection{Could anthocyanins prolong senescence in other plant organs?}

While originally proposed for senescing leaves, my data (chapter 4) show how a prolonged senescence might also be applicable to peduncles and pedicels. Extending this hypothesis further might also explain why the degradation of chlorophyll in other organs often coincides with an accumulation of anthocyanins - in particular, why fruits change colour during maturation. Long seen as a means to attract seed dispersers (Allen, 1879), fruit colour might instead be a by-product of anthocyanin accumulation, produced for a physiological function. The possibility of this co-option is highlighted by the identification of several patterns in fruit colouration, the evolution of which is poorly explained when attributed to frugivore selection. Plant species that invest the most in a fruit's nutritional reward, similarly invest the most in anthocyanins (Schaefer et al., 2008), yet, conspicuousness of bird-dispersed fruits, as seen through models of avian vision, corresponds poorly with anthocyanin content (Schaefer et al., 2008), and many species fail to exploit those colours which are maximally contrasted 
(Schaefer et al., 2007). Anthocyanic colouration in fruits may therefore be under selection pressures beyond those exerted by seed dispersers.

It has long been recognised that fruit ripening shares many characteristics with foliar senescence (Brady, 1987); both involve the catabolism of photosynthetic machinery and both coincide with progression of an abscission zone where the organ attaches to the plant. It might be that anthocyanins produced during fruit maturation provide a function comparable to that proposed for senescing leaves, i.e. anthocyanins may prolong the period for which a fruit is ripe. It has already been demonstrated for tomato that anthocyanin-rich mutants retain skin firmness (a measure of ripeness) for longer and exhibit double the shelf-life as wild-type varieties (Zhang et al., 2013a). Obviously, additional testing is required to show that anthocyanins are responsible for prolonging senescence in peduncles and leaves, let alone fruits, yet application of this hypothesis to other plant organs provides an exciting avenue for future research on anthocyanin function.

\subsection{Conclusion}

A century has passed since Murial Wheldale (1916) published the first review of anthocyanin function in plants. In that time, the number of purported roles for anthocyanins has grown markedly and technology has provided us with a plethora of new ways with which we can test these roles. Regardless, Wheldale (1916) introduced a dichotomy amongst those purported functions (communicative functions were associated with reproductive organs and physiological functions were associated with vegetative organs) and a hundred years on this dichotomy in anthocyanin research still exists. Here, I redressed this imbalance and showed that anthocyanins are responsible for imparting a communicative function in vegetative organs and a physiological function in reproductive organs. In chapter 2, I showed that anthocyanic leaf margins in $P$. colorata provide information on defensive investment and are the trait responsible for deterring herbivory. In chapters 3 and 4, I showed that anthocyanins in Sambucus spp. peduncles are responsible for ameliorating light stress during senescence and that anthocyanins may additionally prolong the senescence period. In doing so, I have shown that this dichotomy in anthocyanin research is unwarranted and unless the significance of that point is acknowledged, we will fail to identify those functions that anthocyanins perform in either reproductive or vegetative organs. Only by identifying those roles which anthocyanins perform can we begin to understand the adaptive significance of anthocyanin production in plants. 


\section{References}

Agati G, Tattini M. 2010. Multiple functional roles of flavonoids in photoprotection. New Phytologist 186: 786-793.

Akhtar Y, Isman MB. 2003. Larval exposure to oviposition deterrents alters subsequent oviposition behavior in generalist, Trichoplusia ni and specialist, Plutella xylostella moths. Journal of Chemical Ecology 29: 1853-1870.

Alexieva V, Sergiev I, Mapelli S, Karanov E. 2001. The effect of drought and ultraviolet radiation on growth and stress markers in pea and wheat. Plant, Cell \& Environment 24: 1337-1344.

Alfenito MR, Souer E, Goodman CD, Buell R, Mol J, Koes R, Walbot V. 1998. Functional complementation of anthocyanin sequestration in the vacuole by widely divergent glutathione S-transferases. The Plant Cell Online 10: 1135-1149.

Allen G. 1879. The colour sense: its origin and development. London: Trübner \& Co.

Andersen ØМ, Jordheim M. 2010. Anthocyanins. eLS DOI: 10.1002/9780470015902.a0001909.pub2

Anderson P, Hilker M, Löfqvist J. 1995. Larval diet influence on oviposition behaviour in Spodoptera littoralis. Entomologia Experimentalis et Applicata 74: 71-82.

Apel K, Hirt H. 2004. Reactive oxygen species: metabolism, oxidative stress, and signal transduction. Annual Review of Plant Biology 55: 373-399.

Archetti M. 2000. The origin of autumn colours by coevolution. Journal of Theoretical Biology 205: 625-630.

Archetti M. 2009a. Classification of hypotheses on the evolution of autumn colours. Oikos 118: 328-333.

Archetti M. 2009b. Evidence from the domestication of apple for the maintenance of autumn colours by coevolution. Proceedings of the Royal Society B: Biological Sciences 276: 2575-2580.

Archetti M. 2009c. Phylogenetic analysis reveals a scattered distribution of autumn colours. Annals of Botany 103: 703-713.

Archetti M, Brown SP. 2004. The coevolution theory of autumn colours. Proceedings of the Royal Society of London. Series B: Biological Sciences 271: 1219-1223.

Archetti M, Döring TF, Hagen SB, Hughes NM, Leather SR, Lee DW, Lev-Yadun S, Manetas Y, Ougham HJ, Schaberg PG et al. 2009. Unravelling the evolution of autumn colours: an interdisciplinary approach. Trends in Ecology \& Evolution 24: 166-173.

Archetti M, Leather SR. 2005. A test of the coevolution theory of autumn colours: colour preference of Rhopalosiphum padi on Prunus padus. Oikos 110: 339-343.

Arreola JA, González AMC, Aguilar LAV, León MTC, Pineda JP, García EA. 2008. Effect of calcium, boron and molybdenum on plant growth and bract pigmentation in poinsettia. Revista Fitotecnia Mexicana 31.

Asada K. 2006. Production and scavenging of reactive oxygen species in chloroplasts and their functions. Plant Physiology 141: 391-396.

Asakawa Y, Dawson GW, Griffiths DC, Lallemand JY, Ley SV, Mori K, Mudd A, Pezechkleclaire M, Pickett JA, Watanabe $\mathrm{H}$ et al. 1988. Activity of drimane antifeedants and related compounds against aphids, and comparative biological effects and chemical reactivity of (-)- polygodial and (+)- polygodial. Journal of Chemical Ecology 14: 1845-1855.

Balakumar T, Vincent VHB, Paliwal K. 1993. On the interaction of UV-B radiation (280$315 \mathrm{~nm}$ ) with water stress in crop plants. Physiologia Plantarum 87: 217-222. 
Balo E, Panczel M, Prileszky G, Gentischer G 1975. The identification of potassium deficiency in peach trees by leaf analysis.In. Proceedings of the 10th Congress of the International Potash Institute held in June 1974 in Budapest, Hungary. Worblaufen, Bern, Switzerland: The Institute, 245-253.

Baranowska M, Krupa Z, Orzol D. 1996. Can anthocyanins be considered as heavy metal stress indicator in higher plants? Acta Physiologiae Plantarum 18: 147-151.

Barbehenn RV, Peter Constabel C. 2011. Tannins in plant-herbivore interactions. Phytochemistry 72: 1551-1565.

Barnes CS, Loder JW. 1962. The structure of polygodial: a new sesquiterpene dialdehyde from Polygonum hydropiper L. Australian Journal of Chemistry 15: 322-327.

Bartosz G. 1997. Oxidative stress in plants. Acta Physiologiae Plantarum 19: 47-64.

Bayly MJ, Kellow AV. 2006. An illustrated guide to New Zealand Hebes. Wellington, NZ: Te Papa press.

Beggs CJ, Wellmann E. 1985. Analysis of light-controlled anthocyanin formation in coleoptiles of Zea mays L.: the role of UV-B, blue, red and far-red light. Photochemistry and Photobiology 41: 481-486.

Bernays EA. 1981. Plant tannins and insect herbivores: an appraisal. Ecological Entomology 6: $353-360$.

Bernays EA. 1998. Evolution of feeding behavior in insect herbivores - Success seen as different ways to eat without being eaten. Bioscience 48: 35-44.

Bernays EA, Cooper-Driver GA, Bilgener M. 1989. Herbivores and plant tannins: Academic Press.

Bilger W, Björkman O. 1990. Role of the xanthophyll cycle in photoprotection elucidated by measurements of light-induced absorbance changes, fluorescence and photosynthesis in leaves of Hedera canariensis. Photosynthesis Research 25: 173185.

Boulton R. 2001. The copigmentation of anthocyanins and its role in the color of red wine: a critical review. American Journal of Enology and Viticulture 52: 67-87.

Bowmaker JK. 1980. Colour vision in birds and the role of oil droplets. Trends in Neurosciences 3: 196-199.

Brady C. 1987. Fruit ripening. Annual review of plant physiology 38: 155-178.

Briscoe AD, Chittka L. 2001. The evolution of color vision in insects. Annual Review of Entomology 46: 471-510.

Burger J, Edwards GE. 1996. Photosynthetic efficiency, and photodamage by UV and visible radiation, in red versus green leaf Coleus varieties. Plant and Cell Physiology 37: 395-399.

Burns KC, Dalen JL. 2002. Foliage color contrasts and adaptive fruit color variation in a bird-dispersed plant community. Oikos 96: 463-469.

Castañeda-Ovando A, Pacheco-Hernández MdL, Páez-Hernández ME, Rodríguez JA, Galán-Vidal CA. 2009. Chemical studies of anthocyanins: a review. Food Chemistry 113: 859-871.

Catalá R, Medina J, Salinas J. 2011. Integration of low temperature and light signaling during cold acclimation response in Arabidopsis. Proceedings of the National Academy of Sciences 108: 16475-16480.

Chalker-Scott L. 1999. Environmental significance of anthocyanins in plant stress responses. Photochemistry and Photobiology 70: 1-9.

Chalker-Scott L 2002. Do anthocyanins function as osmoregulators in leaf tissues? Advances in Botanical Research: Academic Press, 103-127.

Chaves MM, Flexas J, Pinheiro C. 2009. Photosynthesis under drought and salt stress: regulation mechanisms from whole plant to cell. Annals of Botany 103: 551-560. 
Chazdon RL. 1986. The costs of leaf support in understory palms: economy versus safety. The American Naturalist 127: 9-30.

Cheng YJ, Deng XP, Chen W. 2013. Enhanced salt stress tolerance in transgenic potato plants expressing IbMYB1, a sweet potato transcription factor. Journal of microbiology and biotechnology 23: 1737-1746.

Chow JK, Akhtar Y, Isman MB. 2005. The effects of larval experience with a complex plant latex on subsequent feeding and oviposition by the cabbage looper moth: Trichoplusia ni (Lepidoptera: Noctuidae). Chemoecology 15: 129-133.

Christie P, Alfenito M, Walbot V. 1994. Impact of low-temperature stress on general phenylpropanoid and anthocyanin pathways: Enhancement of transcript abundance and anthocyanin pigmentation in maize seedlings. Planta 194: 541-549.

Close DC, McArthur C. 2002. Rethinking the role of many plant phenolics: protection from photodamage not herbivores? Oikos 99: 166-172.

Cooney LJ, van Klink JW, Hughes NM, Perry NB, Schaefer HM, Menzies IJ, Gould KS. 2012. Red leaf margins indicate increased polygodial content and function as visual signals to reduce herbivory in Pseudowintera colorata. New Phytologist 194: 488-497.

Costa D, Galvão AM, Di Paolo RE, Freitas AA, Lima JC, Quina FH, Maçanita AL. 2014. Photochemistry of the hemiketal form of anthocyanins and its potential role in plant protection from UV-B radiation. Tetrahedron.

dela Fuente R, Leopold A. 1968. Senescence processes in leaf abscission. Plant Physiology 43: 1496.

Delgado-Vargas F, Jimenez AR, Paredes-Lopez O. 2000. Natural pigments: carotenoids, anthocyanins, and betalains - characteristics, biosynthesis, processing, and stability. Critical Reviews in Food Science and Nutrition 40: 173-289.

Dell B, Malajczuk N. 1994. Boron deficiency in eucalypt plantations in China. Canadian Journal of Forest Research 24: 2409-2416.

Dicke M, Sabelis MW. 1987. How plants obtain predatory mites as bodyguards. Netherlands Journal of Zoology 38: 148-165.

Dkhil BB, Denden M. 2012. Effect of salt stress on growth, anthocyanins, membrane permeability and chlorophyll fluorescence of Okra (Abelmoschus esculentus L.) seedlings. American Journal of Plant Physiology 7: 174-183.

Dodd IC, Critchley C, Woodall GS, Stewart GR. 1998. Photoinhibition in differently coloured juvenile leaves of Syzygium species. Journal of Experimental Botany 49: 1437-1445.

Dominy NJ, Lucas PW, Ramsden LW, Riba-Hernandez P, Stoner KE, Turner IM. 2002. Why are young leaves red? Oikos 98: 163-176.

Doring TF, Archetti M, Hardie J. 2009. Autumn leaves seen through herbivore eyes. Proceedings of the Royal Society B 276: 121-127.

Doring TF, Chittka L. 2007. Visual ecology of aphids - a critical review on the role of colours in host finding. Arthropod-Plant Interactions 1: 3-16.

Dufoo-Hurtado MD, Zavala-Gutiérrez KG, Cao CM, Cisneros-Zevallos L, GuevaraGonzález RG, Torres-Pacheco I, Vázquez-Barrios ME, Rivera-Pastrana DM, Mercado-Silva EM. 2013. Low-temperature conditioning of "seed" cloves enhances the expression of phenolic metabolism related genes and anthocyanin content in 'coreano' garlic (Allium sativum) during plant development. Journal of Agricultural and Food Chemistry 61: 10439-10446.

Efeoğlu B, Ekmekçi Y, Çiçek N. 2009. Physiological responses of three maize cultivars to drought stress and recovery. South African Journal of Botany 75: 34-42. 
Eryllmaz F. 2006. The relationships between salt stress and anthocyanin content in higher plants. Biotechnology \& Biotechnological Equipment 20: 47-52.

Esteban R, Fernández-Marín B, Becerril JM, García-Plazaola JI. 2008. Photoprotective implications of leaf variegation in E. dens-canis L. and P. officinalis L. Journal of Plant Physiology 165: 1255-1263.

Facelli J. 1993. Experimental evaluation of the foliar flag hypothesis using fruits of Rhus glabra (L.). Oecologia 93: 70-72.

Feild TS, Lee DW, Holbrook NM. 2001. Why leaves turn red in autumn. The role of anthocyanins in senescing leaves of red-osier dogwood. Plant Physiology 127: 566574.

Ferreyra MLF, Rius SP, Casati P. 2012. Flavonoids: biosynthesis, biological functions, and biotechnological applications. Frontiers in plant science 3: 1-15.

Flint-Garcia SA, Thuillet AC, Yu JM, Pressoir G, Romero SM, Mitchell SE, Doebley J, Kresovich S, Goodman MM, Buckler ES. 2005. Maize association population: a high-resolution platform for quantitative trait locus dissection. Plant Journal 44: 1054-1064.

Fondom NY, Castro-Nava S, Huerta AJ. 2009. Photoprotective mechanisms during leaf ontogeny: cuticular development and anthocyanin deposition in two morphs of Agave striata that differ in leaf coloration. Botany 87: 1186-1197.

Foyer CH, Noctor G, van Emden HF. 2007. An evaluation of the costs of making specific secondary metabolites: does the yield penalty incurred by host plant resistance to insects result from competition for resources? International Journal of Pest Management 53: 175-182.

Freschet GT, Cornelissen JHC, van Logtestijn RSP, Aerts R. 2010. Substantial nutrient resorption from leaves, stems and roots in a subarctic flora: what is the link with other resource economics traits? New Phytologist 186: 879-889.

Furuta K. 1986. Host preference and population dynamics in an autumnal population of the maple aphid, Periphyllus californiensis Shinji (Homoptera, Aphididae). Journal of Applied Entomology 102: 93-100.

Garriga M, Retamales JB, Romero-Bravo S, Caligari PDS, Lobos GA. 2014. Chlorophyll, anthocyanin, and gas exchange changes assessed by spectroradiometry in Fragaria chiloensis under salt stress. Journal of Integrative Plant Biology 56: 505515.

Gerard PJ, Perry NB, Ruf LD, Foster LM. 1993. Antifeedant and insecticidal activity of compounds from Pseudowintera colorata (Winteraceae) on the webbing clothes moth, Tineola bisselliella (Lepidoptera, Tineidae) and the Australian carpet beetle, Anthrenocerus australis (Coleoptera, Dermestidae). Bulletin of Entomological Research 83: 547-552.

Gilmore AM, Yamamoto HY. 1991. Resolution of lutein and zeaxanthin using a nonendcapped, lightly carbon-loaded C18 high-performance liquid chromatographic column. Journal of Chromatography A 543: 137-145.

Gitelson AA, Merzlyak MN, Chivkunova OB. 2001. Optical properties and nondestructive estimation of anthocyanin content in plant leavesII. Photochemistry and Photobiology 74: 38-45.

Glińska S, Bartczak M, Oleksiak S, Wolska A, Gabara B, Posmyk M, Janas K. 2007. Effects of anthocyanin-rich extract from red cabbage leaves on meristematic cells of Allium cepa L. roots treated with heavy metals. Ecotoxicology and Environmental Safety 68: 343-350.

Goszczynska D, Zieslin N. 1993. Abscission of flower peduncles in rose (Rosa $\times$ hybrida) plants and evolution of ethylene. Journal of Plant Physiology 142: 214-217. 
Gould KS. 2004. Nature's Swiss army knife: the diverse protective roles of anthocyanins in leaves. Journal of Biomedicine and Biotechnology: 314-320.

Gould KS 2010. Muriel Wheldale Onslow and the rediscovery of anthocyanin function in plants. Recent Advances in Polyphenol Research: Wiley-Blackwell, 206-225.

Gould KS, Davies KM, Winefield CM. 2009. Anthocyanins: biosynthesis, functions and applications. New York: Springer.

Gould KS, Dudle DA, Neufeld HS. 2010. Why some stems are red: cauline anthocyanins shield photosystem II against high light stress. Journal of Experimental Botany 61: 2707-2717.

Gould KS, Kuhn DN, Lee DW, Oberbauer SF. 1995. Why leaves are sometimes red. Nature 378: 241-242.

Gould KS, McKelvie J, Markham KR. 2002a. Do anthocyanins function as antioxidants in leaves? Imaging of $\mathrm{H} 2 \mathrm{O} 2$ in red and green leaves after mechanical injury. Plant Cell and Environment 25: 1261-1269.

Gould KS, Vogelmann TC, Han T, Clearwater MJ. 2002b. Profiles of photosynthesis within red and green leaves of Quintinia serrata. Physiologia Plantarum 116: $127-$ 133.

Gratão PL, Polle A, Lea PJ, Azevedo RA. 2005. Making the life of heavy metal-stressed plants a little easier. Functional Plant Biology 32: 481-494.

Guo Y, Gan S 2005. Leaf senescence: signals, execution, and regulation. In: Gerald PS ed. Current Topics in Developmental Biology: Academic Press, 83-112.

Gutterman Y, Chauser-Volfson E. 2000. The distribution of the phenolic metabolites barbaloin, aloeresin and aloenin as a peripheral defense strategy in the succulent leaf parts of Aloe arborescens. Biochemical Systematics and Ecology 28: 825-838.

Hada H, Hidema J, Maekawa M, Kumagai T. 2003. Higher amounts of anthocyanins and UV-absorbing compounds effectively lowered CPD photorepair in purple rice (Oryza sativa L.). Plant, Cell \& Environment 26: 1691-1701.

Hadagal BN, Manjunath A, Goud JV. 1981. Linkage of genes for anthocyanin pigmentation in rice (Oryza sativa L.). Euphytica 30: 747-754.

Hagen SB, Debeausse S, Yoccoz NG, Folstad I. 2004. Autumn coloration as a signal of tree condition. Proceedings of the Royal Society of London Series B 271: S184-S185.

Hagen SB, Folstad I, Jakobsen SW. 2003. Autumn colouration and herbivore resistance in mountain birch (Betula pubescens). Ecology Letters 6: 807-811.

Hagstrum DW, Subramanyam B. 2010. Immature insects: ecological roles of mobility. American Entomologist 56: 230-241.

Hakala M, Rantamäki S, Puputti E-M, Tyystjärvi T, Tyystjärvi E. 2006. Photoinhibition of manganese enzymes: insights into the mechanism of photosystem II photoinhibition. Journal of Experimental Botany 57: 1809-1816.

Hale KL, McGrath SP, Lombi E, Stack SM, Terry N, Pickering IJ, George GN, PilonSmits EAH. 2001. Molybdenum sequestration in Brassica species. A role for anthocyanins? Plant Physiology 126: 1391-1402.

Hale KL, Tufan HA, Pickering IJ, George GN, Terry N, Pilon M, Pilon-Smits EAH. 2002. Anthocyanins facilitate tungsten accumulation in Brassica. Physiologia Plantarum 116: 351-358.

Hamilton WD, Brown SP. 2001. Autumn tree colours as a handicap signal. Proceedings of the Royal Society of London Series B 268: 1489-1493.

Haque I, Walmsley D. 1973. Response of pak-choi (Brassica chinensis L) to added sulfur in some West Indian soils. Tropical Agriculture 50: 55-61. 
Harris MO, Foster SP, Bittar T, Ekanayake K, Looij K, Howard A. 1995. Visual behaviour of neonate larvae of the light brown apple moth. Entomologia Experimentalis et Applicata 77: 323-334.

Hassouna MG. 1977. Interveinal chlorosis of Bougainvillea spp. in the Sudan due to magnesium deficiency. Egyptian Journal of Phytopathology 7: 59-65.

Hatier J-HB, Clearwater MJ, Gould KS. 2013. The functional significance of blackpigmented leaves: photosynthesis, photoprotection and productivity in Ophiopogon planiscapus 'Nigrescens'. PLoS ONE 8: e67850.

Hatier J-HB, Gould KS 2009. Anthocyanin function in vegetative organs. In: Winefield C, Davies K, Gould K eds. Anthocyanins: biosynthesis, functions and applications. New York: Springer, 1-19.

Hendrickson L, Förster B, Pogson B, Chow W. 2005. A simple chlorophyll fluorescence parameter that correlates with the rate coefficient of photoinactivation of Photosystem II. Photosynthesis Research 84: 43-49.

Hoch WA, Singsaas EL, McCown BH. 2003. Resorption protection. Anthocyanins facilitate nutrient recovery in autumn by shielding leaves from potentially damaging light levels. Plant Physiology 133: 1296-1305.

Hoch WA, Zeldin EL, McCown BH. 2001. Physiological significance of anthocyanins during autumnal leaf senescence. Tree Physiology 21: 1-8.

Holton TA, Cornish EC. 1995. Genetics and biochemistry of anthocyanin biosynthesis. The Plant Cell 7: 1071.

Hora KH, Roessingh P, Menken SBJ. 2005. Inheritance and plasticity of adult host acceptance in Yponomeuta species: implications for host shifts in specialist herbivores. Entomologia Experimentalis et Applicata 115: 271-281.

Hörtensteiner S, Feller U. 2002. Nitrogen metabolism and remobilization during senescence. Journal of Experimental Botany 53: 927-937.

Hughes NM. 2011. Winter leaf reddening in 'evergreen' species. New Phytologist 190: 573581.

Hughes NM, Carpenter KL, Cannon JG. 2013. Estimating contribution of anthocyanin pigments to osmotic adjustment during winter leaf reddening. Journal of Plant Physiology 170: 230-233.

Hughes N, Carpenter K, Keidel T, Miller C, Waters M, Smith W. 2014. Photosynthetic costs and benefits of abaxial versus adaxial anthocyanins in Colocasia esculenta 'Mojito'. Planta DOI: 10.1007/s00425-014-2090-6.

Hughes NM, Morley CB, Smith WK. 2007. Coordination of anthocyanin decline and photosynthetic maturation in juvenile leaves of three deciduous tree species. New Phytologist 175: 675-685.

Hughes NM, Neufeld HS, Burkey KO. 2005. Functional role of anthocyanins in high-light winter leaves of the evergreen herb Galax urceolata. New Phytologist 168: 575-587.

Hughes NM, Reinhardt K, Feild TS, Gerardi AR, Smith WK. 2010a. Association between winter anthocyanin production and drought stress in angiosperm evergreen species. Journal of Experimental Botany.

Hughes NM, Smith WK, Gould KS. 2010b. Red (anthocyanic) leaf margins do not correspond to increased phenolic content in New Zealand Veronica spp. Annals of Botany 105: 647-654.

Hughes NM, Vogelmann TC, Smith WK. 2008. Optical effects of abaxial anthocyanin on absorption of red wavelengths by understorey species: revisiting the back-scatter hypothesis. Journal of Experimental Botany 59: 3435-3442. 
Jarret RL, Vuylsteke DR, Gawel NJ, Pimentel RB, Dunbar LJ. 1993. Detecting genetic diversity in diploid bananas using PCR and primers from a highly repetitive DNA sequence. Euphytica 68: 69-76.

Jiang CD, Li PM, Gao HY, Zou Q, Jiang GM, Li LH. 2005. Enhanced photoprotection at the early stages of leaf expansion in field-grown soybean plants. Plant Science 168: 911-919.

Johanson FD, Walker RB. 1963. Nutrient deficiencies and foliar composition of strawberries. Proceedings of the American Society for Horticultural Science 83: 431439.

Jones LW, Kok B. 1966. Photoinhibition of chloroplast reactions. I. Kinetics and action spectra. Plant Physiology 41: 1037-1043.

Júnior APD, Shimizu MM, Moura JCMS, Catharino RR, Ramos RA, Ribeiro RV, Mazzafera P. 2012. Looking for the physiological role of anthocyanins in the leaves of Coffea arabica. Photochemistry and Photobiology 88: 928-937.

Juvany M, Müller M, Munné-Bosch S. 2013. Photo-oxidative stress in emerging and senescing leaves: a mirror image? Journal of Experimental Botany 64: 3087-3098.

Kaliamoorthy S, Rao AS. 1995. Effect of salinity on anthoycnain accumulation in the root of maize. Indian Journal of Plant Physiology 37: 169-170.

Karageorgou P, Buschmann C, Manetas Y. 2008. Red leaf color as a warning signal against insect herbivory: honest or mimetic? Flora 203: 648-652.

Karageorgou P, Manetas Y. 2006. The importance of being red when young: anthocyanins and the protection of young leaves of Quercus coccifera from insect herbivory and excess light. Tree Physiology 26: 613-621.

Keskitalo J, Bergquist G, Gardeström P, Jansson S. 2005. A cellular timetable of autumn senescence. Plant Physiology 139: 1635-1648.

Kester KM, Peterson SC, Hanson F, Jackson DM, Severson RF. 2002. The roles of nicotine and natural enemies in determining larval feeding site distributions of Manduca sexta L. and Manduca quinquemaculata (Haworth) on tobacco. Chemoecology 12: 1-10.

Kong JM, Chia LS, Goh NK, Chia TF, Brouillard R. 2003. Analysis and biological activities of anthocyanins. Phytochemistry 64: 923-933.

Kong L, Wang F, Feng B, Li S, Si J, Zhang B. 2010. The structural and photosynthetic characteristics of the exposed peduncle of wheat (Triticum aestivum L.): an important photosynthate source for grain-filling. BMC plant biology 10: 141.

Krieger-Liszkay A. 2005. Singlet oxygen production in photosynthesis. Journal of Experimental Botany 56: 337-346.

Kyparissis A, Grammatikopoulos G, Manetas Y. 2007. Leaf morphological and physiological adjustments to the spectrally selective shade imposed by anthocyanins in Prunus cerasifera. Tree Physiology 27: 849-857.

Lan JX, Li AL, Chen CX. 2011. Effect of transient accumulation of anthocyanin on leaf development and photoprotection of Fagopyrum dibotrys mutant. Biologia Plantarum 55: 766-770.

Landi M, Degl'Innocenti E, Pardossi A, Guidi L. 2012. Antioxidant and photosynthetic reponses in plants under boron toxicity: a review. American Journal of Agricultural and Biological Science 7: 255-270.

Landi M, Guidi L, Pardossi A, Tattini M, Gould KS. 2014. Photoprotection by foliar anthocyanins mitigates effects of boron toxicity in sweet basil (Ocimum basilicum). Planta. DOI: 10.1007/s00425-014-2087-1 
Landi M, Pardossi A, Remorini D, Guidi L. 2013. Antioxidant and photosynthetic response of a purple-leaved and a green-leaved cultivar of sweet basil (Ocimum basilicum) to boron excess. Environmental and Experimental Botany 85: 64-75.

Lau TSL, Eno E, Goldstein G, Smith C, Christopher DA. 2006. Ambient levels of UV-B in Hawaii combined with nutrient deficiency decrease photosynthesis in near-isogenic maize lines varying in leaf flavonoids: Flavonoids decrease photoinhibition in plants exposed to UV-B. Photosynthetica 44: 394-403.

Lawler IR, Eschler BM, Schliebs DM, Foley WJ. 1999. Relationship between chemical functional groups on Eucalyptus secondary metabolites and their effectiveness as marsupial antifeedants. Journal of Chemical Ecology 25: 2561-2573.

Lawler IR, Foley WJ, Eschler BM, Pass DM, Handasyde K. 1998. Intraspecific variation in Eucalyptus secondary metabolites determines food intake by folivorous marsupials. Oecologia 116: 160-169.

Lee DW 2002. Anthocyanins in autumn leaf senescence. In: Gould KS, Lee DW eds. Advances in Botanical Research. Amsterdam: Academic Press, 147-165.

Lee DW, Lowry JB, Stone B. 1979. Abaxial anthocyanin layer in leaves of tropical rain forest plants: enhancer of light capture in deep shade. Biotropica: 70-77.

Lee DW, O'Keefe J, Holbrook NM, Feild TS. 2003. Pigment dynamics and autumn leaf senescence in a New England deciduous forest, eastern USA. Ecological Research 18: 677-694.

Lee HY, Hong YN, Chow WS. 2001. Photoinactivation of photosystem II complexes and photoprotection by non-functional neighbours in Capsicum annuum L. leaves. Planta 212: 332-342.

Lepeduš H, Gaća V, Viljevac M, Kovač S, Fulgosi H, Šimić D, Jurković V, Cesar V. 2011. Changes in photosynthetic performance and antioxidative strategies during maturation of Norway maple (Acer platanoides L.) leaves. Plant Physiology and Biochemistry 49: 368-376.

Lev-Yadun S. 2001. Aposematic (warning) coloration associated with thorns in higher plants. Journal of Theoretical Biology 210: 385-388.

Lev-Yadun S. 2003a. Weapon (thorn) automimicry and mimicry of aposematic colorful thorns in plants. Journal of Theoretical Biology 224: 183-188.

Lev-Yadun S. 2003b. Why do some thorny plants resemble green zebras? Journal of Theoretical Biology 224: 483-489.

Lev-Yadun S 2006. Defensive coloration in plants: a review of current ideas about antiherbivore coloration strategies. In: Teixeira da Silva JA ed. Floriculture, ornamental and plant biotechnology: advances and topical issues. London: Global Science Books, 292-299.

Lev-Yadun S 2009a. Aposematic (warning) coloration in plants. In: Baluska F ed. Plantenvironment interactions. From sensory plant biology to active plant behavior. Berlin: Springer-Verlag, 167-202.

Lev-Yadun S. 2009b. Müllerian and Batesian mimicry rings of white-variegated aposematic spiny and thorny plants: A hypothesis. Israel Journal of Plant Sciences 57: 107-116.

Lev-Yadun S. 2009c. Müllerian mimicry in aposematic spiny plants. Plant Signal Behav 4: 482-483.

Lev-Yadun S, Dafni A, Flaishman MA, Inbar M, Izhaki I, Katzir G, Ne'eman G. 2004. Plant coloration undermines herbivorous insect camouflage. BioEssays 26: 11261130.

Lev-Yadun S, Gould KS. 2007. What do red and yellow autumn leaves signal? Botanical Review 73: 279-289. 
Lev-Yadun S, Gould KS 2009. Role of anthocyanins in plant defense. In: Gould KS, Davies $\mathrm{KM}$, Winefield CM eds. Life's colorful solutions: the biosynthesis, functions, and applications of anthocyanins. Berlin: Springer-Verlag, 21-48.

Lev-Yadun S, Holopainen JK. 2009. Why red-dominated American autumn leaves and yellow-dominated autumn leaves in Northern Europe? New Phytologist 183: 506-512.

Lev-Yadun S, Inbar M, Izhaki I, Ne'eman G, Dafni A. 2002. Colour patterns in vegetative parts of plants deserve more research attention. Trends in Plant Science 7: 59-60.

Lev-Yadun S, Ne'eman G, Izhaki I. 2009. Unripe red fruits may be aposematic. Plant Signaling \& Behavior 4: 836-841.

Levey DJ. 1990. Habitat-dependent fruiting behaviour of an understorey tree, Miconia centrodesma, and tropical treefall gaps as keystone habitats for frugivores in Costa Rica. Journal of Tropical Ecology 6: 409-420.

Li P, Cheng L. 2008. The shaded side of apple fruit becomes more sensitive to photoinhibition with fruit development. Physiologia Plantarum 134: 282-292.

Li X, Baskin JM, Baskin CC. 1999. Contrasting dispersal phenologies in two fleshy-fruited congeneric shrubs, Rhus aromatica Ait. and Rhus glabra L. (Anacardiaceae). Canadian Journal of Botany 77: 976-988.

Liakopoulos G, Nikolopoulos D, Klouvatou A, Vekkos K, Manetas Y, Karabourniotis G. 2006. The photoprotective role of epidermal anthocyanins and surface pubescence in young leaves of grapevine (Vitis vinifera). Annals of Botany 98: 257-265.

Liakopoulos G, Spanorigas I. 2012. Foliar anthocyanins in Pelargonium $\times$ hortorum are unable to alleviate light stress under photoinhibitory conditions. Photosynthetica 50: 254-262.

Lichtenthaler H. 1987. Chlorophyll and carotenoids: pigments of photosynthetic biomembranes. Methods in Enzymology 148: 350-385.

Lim PO, Kim HJ, Gil Nam H. 2007. Leaf Senescence. Annual Review of Plant Biology 58: 115-136.

Logan BA, Demmig-Adams B, Adams WW. 1998. Antioxidants and xanthophyll cycledependent energy dissipation in Cucurbita pepo L. and Vinca major L. upon a sudden increase in growth PPFD in the field. Journal of Experimental Botany 49: 1881-1888.

Long SP, Humphries S, Falkowski PG. 1994. Photoinhibition of photosynthesis in nature. Annual Review of Plant Physiology and Plant Molecular Biology 45: 633-662.

Løvdal T, Olsen KM, Slimestad R, Verheul M, Lillo C. 2010. Synergetic effects of nitrogen depletion, temperature, and light on the content of phenolic compounds and gene expression in leaves of tomato. Phytochemistry 71: 605-613.

Lyons RE, Widmer RE. 1984. A gibberellin-auxin synergism associated with peduncle elongation and senescence in Cyclamen persicum Mill. 'swan lake'. Botanical Gazette 145: $170-175$.

Maayan I, Shaya F, Ratner K, Mani Y, Lavee S, Avidan B, Shahak Y, OstersetzerBiran O. 2008. Photosynthetic activity during olive (Olea europaea) leaf development correlates with plastid biogenesis and rubisco levels. Physiologia Plantarum 134: 547-558.

Manetas Y. 2006. Why some leaves are anthocyanic and why most anthocyanic leaves are red? Flora - Morphology, Distribution, Functional Ecology of Plants 201: 163-177.

Manetas Y, Buschmann C. 2011. The interplay of anthocyanin biosynthesis and chlorophyll catabolism in senescing leaves and the question of photosystem II photoprotection. Photosynthetica 49: 515-522.

Manetas Y, Drinia A, Petropoulou Y. 2002. High contents of anthocyanins in young leaves are correlated with low pools of xanthophyll cycle components and low risk of photoinhibition. Photosynthetica 40: 349-354. 
Manetas Y, Petropoulou Y, Psaras GK, Drinia A. 2003. Exposed red (anthocyanic) leaves of Quercus coccifera display shade characteristics. Functional Plant Biology 30: 265270.

Markwick NP, Poulton J, Espley RV, Rowan DD, McGhie TK, Wadasinghe G, Wohlers M, Jia Y, Allan AC. 2013. Red-foliaged apples affect the establishment, growth, and development of the light brown apple moth, Epiphyas postvittana. Entomologia Experimentalis et Applicata 146: 261-275.

May JD, Killingbeck KT. 1992. Effects of preventing nutrient resorption on plant fitness and foliar nutrient dynamics. Ecology 73: 1868-1878.

McCallion RF, Cole A, Walker J, Blunt J, Munro M. 1982. Antibiotic substances from New Zealand plants. Planta medica 44: 134-138.

Mendez M, Jones DG, Manetas Y. 1999. Enhanced UV-B radiation under field conditions increases anthocyanin and reduces the risk of photoinhibition but does not affect growth in the carnivorous plant Pinguicula vulgaris. New Phytologist 144: 275-282.

Menzies IJ. 2013. Do foliar anthocyanin pigments in horopito (Pseudowintera colorata) function as visual signals to deter insect herbivores? $\mathrm{PhD}$ Thesis. Victoria University of Wellington Wellington.

Merilaita S, Ruxton GD. 2007. Aposematic signals and the relationship between conspicuousness and distinctiveness. Journal of Theoretical Biology 245: 268-277.

Merzlyak MN, Chivkunova OB, Solovchenko AE, Naqvi KR. 2008. Light absorption by anthocyanins in juvenile, stressed, and senescing leaves. Journal of Experimental Botany 59: 3903-3911.

Meyer GA. 1993. A comparison of the impacts of leaf- and sap-feeding insects on growth and allocation of goldenrod. Ecology 74: 1101-1116.

Michaeli R, Philosoph-Hadas S, Riov J, Meir S. 1999a. Chilling-induced leaf abscission of Ixora coccinea plants. I. Induction by oxidative stress via increased sensitivity to ethylene. Physiologia Plantarum 107: 166-173.

Michaeli R, Riov J, Philosoph-Hadas S, Meir S. 1999b. Chilling-induced leaf abscission of Ixora coccinea plants. II. Alteration of auxin economy by oxidative stress. Physiologia Plantarum 107: 174-180.

Misyura M, Colasanti J, Rothstein SJ. 2012. Physiological and genetic analysis of Arabidopsis thaliana anthocyanin biosynthesis mutants under chronic adverse environmental conditions. Journal of Experimental Botany.

Møller IM, Sweetlove LJ. 2010. ROS signalling - specificity is required. Trends in Plant Science 15: 370-374.

Murata N, Takahashi S, Nishiyama Y, Allakhverdiev SI. 2007. Photoinhibition of photosystem II under environmental stress. Biochimica et Biophysica ActaBioenergetics 1767: 414-421.

Neill S, Gould KS. 2000. Optical properties of leaves in relation to anthocyanin concentration and distribution. Canadian Journal of Botany 77: 1777-1782.

Newsham KK, Geissler PA, Nicolson MJ, Peat HJ, Lewis-Smith RI. 2005. Sequential reduction of UV-B radiation in the field alters the pigmentation of an Antarctic leafy liverwort. Environmental and Experimental Botany 54: 22-32.

Nick P, Ehmann B, Furuya M, Schafer E. 1993. Cell communication, stochastic cell responses, and anthocyanin pattern in mustard cotyledons. Plant Cell 5: 541-552.

Nielsen SL, Simonsen AM. 2011. Photosynthesis and photoinhibition in two differently coloured varieties of Oxalis triangularis - the effect of anthocyanin content. Photosynthetica 49: 346-352. 
Nikiforou C, Manetas Y. 2010. Strength of winter leaf redness as an indicator of stress vulnerable individuals in Pistacia lentiscus. Flora - Morphology, Distribution, Functional Ecology of Plants 205: 424-427.

Nishihara M, Nakatsuka T. 2011. Genetic engineering of flavonoid pigments to modify flower color in floricultural plants. Biotechnology Letters 33: 433-441.

Nishiyama Y, Allakhverdiev SI, Murata N. 2006. A new paradigm for the action of reactive oxygen species in the photoinhibition of photosystem II. Biochimica et Biophysica Acta (BBA) - Bioenergetics 1757: 742-749.

Nishiyama Y, Yamamoto H, Allakhverdiev SI, Inaba M, Yokota A, Murata N. 2001. Oxidative stress inhibits the repair of photodamage to the photosynthetic machinery. The EMBO Journal 20: 5587-5594.

Nissim-Levi A, Kagan S, Ovadia R, Oren-Shamir M. 2003. Effects of temperature, UVlight and magnesium on anthocyanin pigmentation in cocoplum leaves. Journal of horticultural science \& biotechnology 78: 61-64.

Nogués S, Baker NR. 2000. Effects of drought on photosynthesis in Mediterranean plants grown under enhanced UV-B radiation. Journal of Experimental Botany 51: 13091317.

Nyborg M, Hoyt PB. 1970. Boron deficiency in turnip rape grown on gray wooded soils. Canadian Journal of Soil Science 50: 87-88.

Olsson POC, Anderbrant O, Lofstedt C. 2006. Experience influences oviposition behaviour in two pyralid moths, Ephestia cautella and Plodia interpunctella. Animal Behaviour 72: 545-551.

Otte D. 1974. Effects and functions in the evolution of signaling systems. Annual Review of Ecology and Systematics 5: 385-417.

Padhye S, Kambara T, Hendrickson DN, Govindjee. 1986. Manganese-histidine cluster as the functional center of the water oxidation complex in photosynthesis. Photosynthesis Research 9: 103-112.

Page J, Towers N. 2002. Anthocyanins protect light-sensitive thiarubrine phototoxins. Planta 215: 478-484.

Peng C, Lin Z, Lin G, Chen S. 2006. The anti-photooxidation of anthocyanins-rich leaves of a purple rice cultivar. Science in China Series C: Life Sciences 49: 543-551.

Perry NB, Burgess EJ, Lorimer SD, vanKlink JW. 1996a. Fatty acid anilides as internal standards for high performance liquid chromatographic analyses of Valeriana officinalis L and other medicinal plants. Phytochemical Analysis 7: 263-268.

Perry NB, Foster LM, Lorimer SD. 1996b. Infraspecific variation of insecticidal sesquiterpene dialdehydes in Pseudowintera colorata. Phytochemistry 43: 1201-1203.

Perry NB, Gould KS. 2010. Hot chemistry from horopito. Chemistry in New Zealand October: $145-148$.

Peters DJ, Constabel CP. 2002. Molecular analysis of herbivore-induced condensed tannin synthesis: cloning and expression of dihydroflavonol reductase from trembling aspen (Populus tremuloides). The Plant Journal 32: 701-712.

Pfündel E, Ben Ghozlen N, Meyer S, Cerovic Z. 2007. Investigating UV screening in leaves by two different types of portable UV fluorimeters reveals in vivo screening by anthocyanins and carotenoids. Photosynthesis Research 93: 205-221.

Pietrini F, Iannelli M, Massacci A. 2002. Anthocyanin accumulation in the illuminated surface of maize leaves enhances protection from photo-inhibitory risks at low temperature, without further limitation to photosynthesis. Plant, Cell \& Environment 25: 1251-1259.

Pollastri S, Tattini M. 2011. Flavonols: old compounds for old roles. Annals of Botany. DOI: $10.1093 / \mathrm{aob} / \mathrm{mcr} 234$ 
Posmyk MM, Kontek R, Janas KM. 2009. Antioxidant enzymes activity and phenolic compounds content in red cabbage seedlings exposed to copper stress. Ecotoxicology and Environmental Safety 72: 596-602.

Poulton EB. 1890. The colours of animals: their meaning and use especially considered in the case of insects. London: Keegan Paul, Trench, Trubner \& Co.

Pourcel L, Bohorquez-Restrepo A, Irani NG, Grotewold E. 2012. Anthocyanin biosynthesis, regulation, and transport: new insights from model species. Recent Advances in Polyphenol Research, Vol 3 3: 143-160.

Qin FF, Du FL, Xu RY, Xu QC, Tian CM, Li FM, Wang FH. 2009. Photosynthesis in different parts of a wheat plant. Journal of Food, Agriculture \& Environment 7: 399404.

Qiu YT, van Loon JJA, Roessingh P. 1998. Chemoreception of oviposition inhibiting terpenoids in the diamondback moth Plutella xylostella. Entomologia Experimentalis et Applicata 87: 143-155.

Raese JT. 2002. Phosphorus deficiency symptoms in leaves of apple and pear trees as influenced by available soil phosphorus. Communications in Soil Science and Plant Analysis 33: 461-477.

Ralph PJ, Gademann R. 2005. Rapid light curves: A powerful tool to assess photosynthetic activity. Aquatic Botany 82: 222-237.

Ranjan S, Singh R, Singh M, Pathre UV, Shirke PA. 2014. Characterizing photoinhibition and photosynthesis in juvenile-red versus mature-green leaves of Jatropha curcas L. Plant Physiology and Biochemistry 79: 48-59.

Rasheed R, Ashraf MA, Parveen S, Iqbal M, Hussain I. 2014. Effect of salt stress on different growth and biochemical attributes in two canola (Brassica napus L.) cultivars. Communications in Soil Science and Plant Analysis 45: 669-679.

Rautio P, Markkola A, Martel J, Tuomi J, Harma E, Kuikka K, Siitonen A, Riesco IL, Roitto M. 2002. Developmental plasticity in birch leaves: defoliation causes a shift from glandular to nonglandular trichomes. Oikos 98: 437-446.

Redden KM. 2008. A new species of Paloue (Leguminosae: Caesalpinioideae: Detarieae) from Guyana, South America. Brittonia 60: 257-260.

Reeves JL. 2011. Vision should not be overlooked as an important sensory modality for finding host plants. Environmental Entomology 40: 855-863.

Ridley HN. 1930. The dispersal of plants throughout the world. Ashford, England: Reeve.

Roberts JA, Elliott KA, Gonzalez-Carranza ZH. 2002. Abscission, dehiscence, and other cell separation processes. Annual Review of Plant Biology 53: 131-158.

Roberts JA, Whitelaw CA, Gonzalez-Carranza ZH, McManus MT. 2000. Cell separation processes in plants - Models, mechanisms and manipulation. Annals of Botany 86: 223-235.

Rolshausen G, Schaefer HM. 2007. Do aphids paint the tree red (or yellow) — can herbivore resistance or photoprotection explain colourful leaves in autumn? Plant Ecology 191: 77-84.

Rubino DL, McCarthy BC. 2004. Presence of aposematic (warning) coloration in vascular plants of southeastern Ohio. Journal of the Torrey Botanical Society 131: 252-256.

Ruelland E, Vaultier M-N, Zachowski A, Hurry V 2009. Cold signalling and cold acclimation in plants. In: Jean-Claude K, Michel D eds. Advances in Botanical Research: Academic Press, 35-150.

Sabharwal P, Doležel J. 1993. Interspecific hybridization in Brassica: Application of flow cytometry for analysis of ploidy and genome composition in hybrid plants. Biologia Plantarum 35: 169-177. 
Schaberg PG, Murakami PF, Turner MR, Heitz HK, Hawley GJ. 2008. Association of red coloration with senescence of sugar maple leaves in autumn. Trees-Structure and Function 22: 573-578.

Schaberg PG, van den Berg AK, Murakami PF, Shane JB, Donnelly JR. 2003. Factors influencing red expression in autumn foliage of sugar maple trees. Tree Physiology 23: $325-333$.

Schaefer H, Schaefer V. 2007. The evolution of visual fruit signals: concepts and constraints. Seed dispersal: theory and its application in a changing world. Wallingford, UK: CAB International: 59-77.

Schaefer HM. 2011. Why fruits go to the dark side. Acta Oecologica 37: 604-610.

Schaefer HM, Braun J. 2009. Reliable cues and signals of fruit quality are contingent on the habitat in black elder (Sambucus nigra). Ecology 90: 1564-1573.

Schaefer HM, McGraw K, Catoni C. 2008. Birds use fruit colour as honest signal of dietary antioxidant rewards. Functional Ecology 22: 303-310.

Schaefer HM, Rolshausen G. 2006. Plants on red alert: do insects pay attention? BioEssays 28: $65-71$.

Schaefer HM, Rolshausen G. 2007. Aphids do not attend to leaf colour as visual signal, but to the handicap of reproductive investment. Biology Letters 3: 1-4.

Schaefer HM, Ruxton GD. 2011. Plant-animal communication. New York: Oxford University Press.

Schaefer HM, Schaefer V, Vorobyev M. 2007. Are fruit colors adapted to consumer vision and birds equally efficient in detecting colorful signals? The American Naturalist 169: S159-S169.

Schaefer HM, Wilkinson DM. 2004. Red leaves, insects and coevolution: a red herring? Trends in Ecology \& Evolution 19: 616-618.

Shao L, Shu Z, Peng C, Lin Z, Yang C, Gu Q. 2008. Enhanced sensitivity of Arabidopsis anthocyanin mutants to photooxidation: a study with fluorescence imaging. Functional Plant Biology 35: 714-724.

Sherwin H, Farrant J. 1998. Protection mechanisms against excess light in the resurrection plants Craterostigma wilmsii and Xerophyta viscosa. Plant Growth Regulation 24: 203-210.

Shichijo C, Hamada T, Hiraoka M, Johnson C, Hashimoto T. 1993. Enhancement of redlight-induced anthocyanin synthesis in sorghum first internodes by moderate low temperature given in the pre-irradiation culture period. Planta 191: 238-245.

Shroff R, Vergara F, Muck A, Svatos A, Gershenzon J. 2008. Nonuniform distribution of glucosinolates in Arabidopsis thaliana leaves has important consequences for plant defense. Proceedings of the National Academy of Sciences USA 105: 6196-6201.

Singh AK, Saxena KN. 2004. Attraction of larvae of the armyworm Spodoptera litura (Lepidoptera: Noctuidae) to coloured surfaces. European Journal of Entomology 101: 697-699.

Singh P. 1983. A general purpose laboratory diet mixture for rearing insects. Insect Science and Its Application 4: 357-362.

Smith A. 1909. On the internal temperature of leaves in tropical insolation, with special reference to the effect of their colour on the temperature; also observations on the periodicity of the appearance of young coloured leaves of trees growing in Peradeniya gardens. Annals of the Royal Botanical Gardens, Peradeniya 5: 229-298.

Solangaarachchi SM, Gould KS. 2001. Anthocyanin pigmentation in the adventitious roots of Metrosideros excelsa (Myrtaceae). New Zealand Journal of Botany 39: 161-166. 
Solecka D, Boudet A-M, Kacperska A. 1999. Phenylpropanoid and anthocyanin changes in low-temperature treated winter oilseed rape leaves. Plant Physiology and Biochemistry 37: 491-496.

Solovchenko AE, Chivkunova OB. 2011. Physiological role of anthocyanin accumulation in common hazel juvenile leaves. Russian Journal of Plant Physiology 58: 674-680.

Sperdouli I, Moustakas M. 2012. Interaction of proline, sugars, and anthocyanins during photosynthetic acclimation of Arabidopsis thaliana to drought stress. Journal of Plant Physiology 169: 577-585.

Steyn WJ 2009. Prevalence and functions of anthocyanins in fruits. In: Winefield C, Davies K, Gould K eds. Anthocyanins: biosynthesis, functions and applications. New York: Springer, 86-105.

Steyn WJ, Wand SJE, Holcroft DM, Jacobs G. 2002. Anthocyanins in vegetative tissues: a proposed unified function in photoprotection. New Phytologist 155: 349-361.

Steyn WJ, Wand SJE, Jacobs G, Rosecrance RC, Roberts SC. 2009. Evidence for a photoprotective function of low-temperature-induced anthocyanin accumulation in apple and pear peel. Physiologia Plantarum 136: 461-472.

Stiles EW. 1982. Fruit flags: two hypotheses. American naturalist 120: 500.

Stintzing FC, Carle R. 2004. Functional properties of anthocyanins and betalains in plants, food, and in human nutrition. Trends in Food Science \& Technology 15: 19-38.

Stournaras KE, Lo E, Böhning-Gaese K, Cazetta E, Matthias Dehling D, Schleuning M, Stoddard MC, Donoghue MJ, Prum RO, Martin Schaefer H. 2013. How colorful are fruits? Limited color diversity in fleshy fruits on local and global scales. New Phytologist 198: 617-629.

Tahkokorpi M, Taulavuori K, Laine K, Taulavuori E. 2007. After-effects of droughtrelated winter stress in previous and current year stems of Vaccinium myrtillus L. Environmental and Experimental Botany 61: 85-93.

Takahashi A, Takeda K, Ohnishi T. 1991. Light-induced anthocyanin reduces the extent of damage to DNA in UV-irradiated Centaurea cyanus cells in culture. Plant and Cell Physiology 32: 541-547.

Takahashi S, Badger MR. 2011. Photoprotection in plants: a new light on photosystem II damage. Trends in Plant Science 16: 53-60.

Takahashi S, Milward SE, Yamori W, Evans JR, Hillier W, Badger MR. 2010. The solar action spectrum of photosystem II damage. Plant Physiology 153: 988-993.

Takahashi S, Murata N. 2005. Interruption of the Calvin cycle inhibits the repair of photosystem II from photodamage. Biochimica et Biophysica Acta (BBA) Bioenergetics 1708: 352-361.

Takahashi S, Murata N. 2006. Glycerate-3-phosphate, produced by CO2 fixation in the Calvin cycle, is critical for the synthesis of the D1 protein of photosystem II. Biochimica et Biophysica Acta (BBA) - Bioenergetics 1757: 198-205.

Takahashi S, Murata N. 2008. How do environmental stresses accelerate photoinhibition? Trends in Plant Science 13: 178-182.

Tanino KK, Cherry KM, Kriger JN, Hrycan W, Marufu G, Thomas JD, Gray GR. 2014. Photosynthetic responses to temperature-mediated dormancy induction in contrasting ecotypes of red-osier dogwood (Cornus sericea L.). Environmental and Experimental Botany. DOI: 10.1016/j.envexpbot.2014.02.015

Tattini M, Landi M, Brunetti C, Giordano C, Remorini D, Gould KS, Guidi L. 2014. Epidermal coumaroyl anthocyanins protect sweet basil against excess light stress: multiple consequences of light attenuation. Physiologia Plantarum DOI: $10.1111 /$ ppl.12201 
Thorne GN. 1963. Varietal differences in photosynthesis of ears and eaves of barley. Annals of Botany 27: 155-174.

Thorne GN. 1965. Photosynthesis of ears and flag leaves of wheat and barley. Annals of Botany 29: 317-329.

Triantaphylidès C, Krischke M, Hoeberichts FA, Ksas B, Gresser G, Havaux M, Van Breusegem F, Mueller MJ. 2008. Singlet oxygen is the major reactive oxygen species involved in photooxidative damage to plants. Plant Physiology 148: 960-968.

Tsurunaga Y, Takahashi T, Katsube T, Kudo A, Kuramitsu O, Ishiwata M, Matsumoto S. 2013. Effects of UV-B irradiation on the levels of anthocyanin, rutin and radical scavenging activity of buckwheat sprouts. Food Chemistry 141: 552-556.

Tyystjärvi E. 2008. Photoinhibition of photosystem II and photodamage of the oxygen evolving manganese cluster. Coordination Chemistry Reviews 252: 361-376.

Tyystjärvi E, Aro EM. 1996. The rate constant of photoinhibition, measured in lincomycintreated leaves, is directly proportional to light intensity. Proceedings of the National Academy of Sciences 93: 2213-2218.

Van Bael SA, Brawn JD, Robinson SK. 2003. Birds defend trees from herbivores in a Neotropical forest canopy. Proceedings of the National Academy of Sciences 100: 8304-8307.

Varela FJ, Palacios AG, Goldsmith TH 1993. Color vision of birds. In: Zeigler HP, Bischof H-J eds. Vision, brain, and behavior in birds. Cambridge, MA, US: The MIT Press, 77-98.

Veberic R, Jakopic J, Stampar F, Schmitzer V. 2009. European elderberry (Sambucus nigra L.) rich in sugars, organic acids, anthocyanins and selected polyphenols. Food Chemistry 114: 511-515.

Versieux LM, Wanderley MD. 2007. Two new species of Alcantarea (Bromeliaceae: Tillandsioideae) from Brazil. Brittonia 59: 57-64.

Walker LC. 1956. Foliage symptoms as indicators of potassium-deficient soils. Forest Science 2: 113-120.

Wang H, Cao G, Prior RL. 1997. Oxygen radical absorbing capacity of anthocyanins. Journal of Agricultural and Food Chemistry 45: 304-309.

Wang L, Li X, Zhao Q, Jing S, Chen S, Yuan H. 2009. Identification of genes induced in response to low-temperature treatment in tea leaves. Plant Molecular Biology Reporter 27: 257-265.

Wang Y, Zhou B, Sun M, Li Y, Kawabata S. 2012. UV-A light induces anthocyanin biosynthesis in a manner distinct from synergistic blue + UV-B light and UV-A/blue light responses in different parts of the hypocotyls in turnip seedlings. Plant and Cell Physiology 53: 1470-1480.

Wayman KA, de Lange PJ, Larsen L, Sansom CE, Perry NB. 2010. Chemotaxonomy of Pseudowintera: Sesquiterpene dialdehyde variants are species markers. Phytochemistry 71: 766-772.

Webb C, Sykes W, Garnock-Jones P. 1988. Flora of New Zealand, vol. IV. Botany Division, DSIR, Christchurch.

Wheldale M. 1916. The anthocyanin pigments of plants. Cambridge, UK: Cambridge University Press.

White A, Critchley C. 1999. Rapid light curves: a new fluorescence method to assess the state of the photosynthetic apparatus. Photosynthesis Research 59: 63-72.

Williams EL, Hovenden MJ, Close DC. 2003. Strategies of light energy utilisation, dissipation and attenuation in six co-occurring alpine heath species in Tasmania. Functional Plant Biology 30: 1205-1218. 
Willson MF, Whelan CJ. 1990. The evolution of fruit color in fleshy-fruited plants. The American Naturalist 136: 790-809.

Wingler A, Purdy S, MacLean JA, Pourtau N. 2006. The role of sugars in integrating environmental signals during the regulation of leaf senescence. Journal of Experimental Botany 57: 391-399.

Wong AR, Srivastava DS. 2010. Red abaxial colouring reduces herbivory in Columnea consanguinea. Ecotropica 16: 93-99.

Woodall GS, Stewart GR. 1998. Do anthocyanins play a role in UV protection of the red juvenile leaves of Syzygium? Journal of Experimental Botany 49: 1447-1450.

Wu X, Gu L, Prior RL, McKay S. 2004. Characterization of anthocyanins and proanthocyanidins in some cultivars of Ribes, Aronia, and Sambucus and their antioxidant capacity. Journal of Agricultural and Food Chemistry 52: 7846-7856.

Yachandra VK, Sauer K, Klein MP. 1996. Manganese cluster in photosynthesis: where plants oxidize water to dioxygen. Chemical Reviews 96: 2927-2950.

Yasui H, Fukaya M, Wakamura S. 2006. Behavioral responses in feeding to green color as visual stimulus with two lepidopteran larvae, Spodoptera litura (Fabricius) (Noctuidae) and Milionia basalis pryeri Druce (Geometridae). Applied Entomology and Zoology 41: 41-47.

Zeliou K, Manetas Y, Petropoulou Y. 2009. Transient winter leaf reddening in Cistus creticus characterizes weak (stress-sensitive) individuals, yet anthocyanins cannot alleviate the adverse effects on photosynthesis. Journal of Experimental Botany 60: 3031-3042.

Zhang KM, Wang XM, Cui JX, Ogweno JO, Shi K, Zhou YH, Yu JQ. 2011. Characteristics of gas exchange and chlorophyll fluorescence in red and green leaves of Begonia semperflorens. Biologia Plantarum 55: 361-364.

Zhang KM, Yu HJ, Shi K, Zhou YH, Yu JQ, Xia XJ. 2010. Photoprotective roles of anthocyanins in Begonia semperflorens. Plant Science 179: 202-208.

Zhang Q, Su LJ, Chen JW, Zeng XQ, Sun BY, Peng CL. 2012. The antioxidative role of anthocyanins in Arabidopsis under high-irradiance. Biologia Plantarum 56: 97-104.

Zhang Y, Butelli E, De Stefano R, Schoonbeek HJ, Magusin A, Pagliarani C, Wellner N, Hill L, Orzaez D, Granell A et al. 2013a. Anthocyanins double the shelf life of tomatoes by delaying overripening and reducing susceptibility to gray mold. Current Biology 23: 1094-1100.

Zhang YJ, Yang QY, Lee DW, Goldstein G, Cao KF. 2013b. Extended leaf senescence promotes carbon gain and nutrient resorption: importance of maintaining winter photosynthesis in subtropical forests. Oecologia 173: 721-730.

Zimmermann P, Zentgraf U. 2005. The correlation between oxidative stress and leaf senescence during plant development. Cellular and Molecular Biology Letters 10: 515. 